\title{
A Systems Level Analysis of \\ Neuronal Network Function in the Olfactory Bulb: \\ Coding, Connectivity, and Modular organization
}

\author{
PhD Thesis \\ in partial fulfilment of the requirements for the degree \\ "Doctor of Philosophy $(\mathrm{PhD}) / \mathrm{Dr}$. rer. nat." \\ in the Neuroscience Program at the Georg August University Göttingen, \\ Faculty of Biology
}

submitted by

Tsai-Wen Chen

born in

Taipei, Taiwan

Göttingen, 2008 

Advisor, member of $\mathrm{PhD}$ committee:

Member of $\mathrm{PhD}$ committee:

Member of PhD committee:

Date of submission of the $\mathrm{PhD}$ thesis:
Prof. Dr. Dr. Detlev Schild

Prof. Dr. Walter Stuehmer

Dr. Fred Wolf

March 20, 2008 

I hereby declare that I prepared the PhD thesis "A Systems Level Analysis of Neuronal Network Function in the Olfactory Bulb: Coding, Connectivity, and Modular organization" on my own and with no other sources and aids than quoted.

Göttingen, March 20, 2008

Tsai-Wen Chen 

謹將本論文獻給我最摰愛的爸媽, 摘宇, 與貝容

你們對我毫無保留的愛與支持是我一路走來最大的動力

謝謝你們！ 



\section{Table of contents}

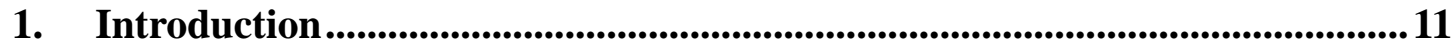

1.1 Neuroscience from a systems point of view ............................................. 11

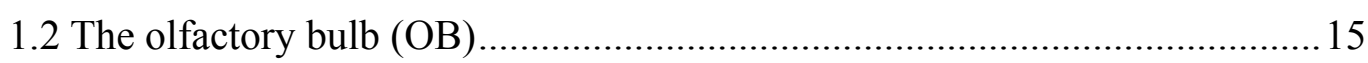

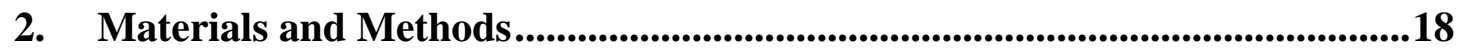

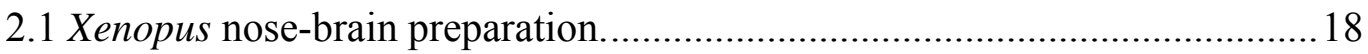

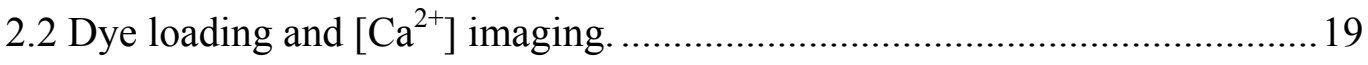

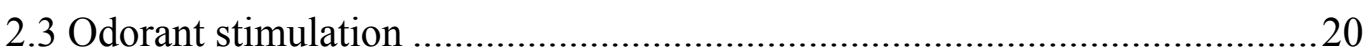

2.4 Electrophysiology and morphological reconstructions...............................20

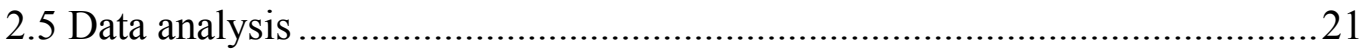

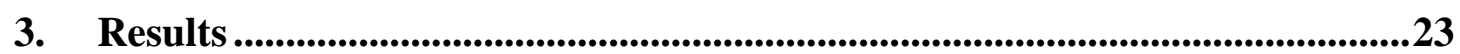

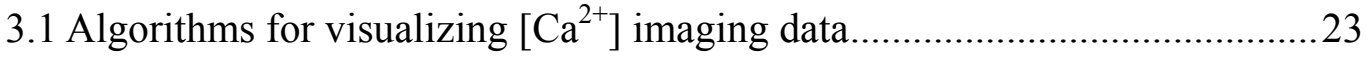

3.1.1 Neighborhood Correlation Map .....................................................23

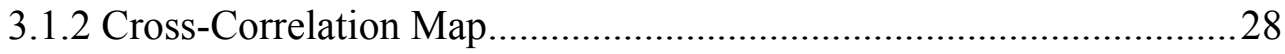

3.2 Correlation structures of OB spontaneous activity ......................................... 34

3.2.1 Identification of synchronous neuronal modules............................... 35

3.2.2 High temporal resolution analysis of synchronous activity............... 41

3.3 Connections of synchronous neurons into the same glomerulus ...................44

3.3.1 Evidences from tracer injection ....................................................... 44

3.3.1 Evidence from cross-correlation maps................................................51

3.4 Characterization of sensory responses in synchronous neurons ....................54

3.4.1 General properties of odor responses in the OB ...............................54

3.4.2 Odor responses in synchronous and non-synchronous neurons. ........56

3.5 Synaptic mechanisms underlying correlated activity ..................................64

3.5.1 Characterization of synaptic inputs and functional coupling..............64

3.5.2 The effect of mutual coupling between synchronous neurons............67

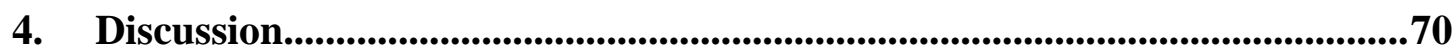

4.1 Correlation maps for visualizing $\left[\mathrm{Ca}^{2+}\right]$ imaging data ............................... 70

4.2 Spontaneous activity in the olfactory bulb............................................... 75

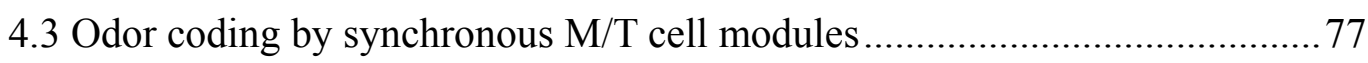

4.3.1 Odor responses in glomerulus-specific neurons ............................... 77

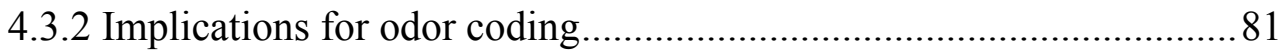

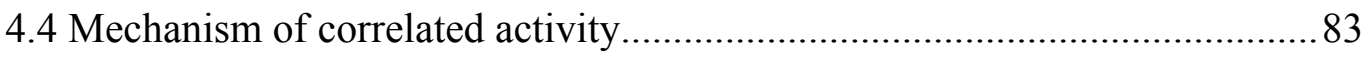

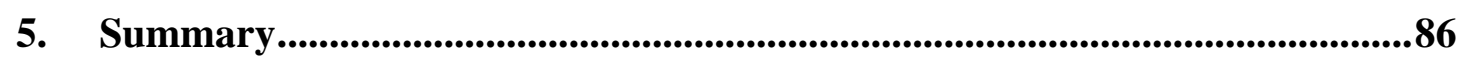

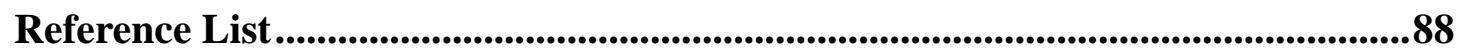

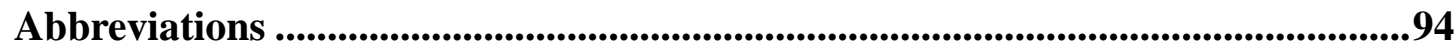

Acknowledgements .....................................................................................................95

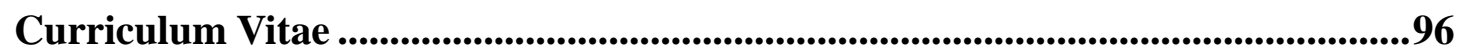





\section{Introduction}

\subsection{Neuroscience from a systems point of view}

The development of modern scientific thinking during the past 300 years is strongly dominated by the principle of reductionism. With an attempt to explain natural phenomena by decomposing them into ever smaller parts, the reductionist approach has met with great success in many scientific disciplines. We can now explain the thermodynamic properties of gases by analyzing the kinetic motions of their constituent molecules. We can understand the metabolism of a complex organism in terms of various chemical reactions, which can further be explained by the quantum mechanical properties of individual atoms and electrons.

In the past century, we saw a similar success of a reductionist approach to the understanding of brain function. We now know that the brain is made up by billions of neurons intricately connected via tiny and highly specialized structures called synapses. We can explain the once enigmatic neuronal electrical discharges by the current flows via individual ion channels located on neurons' membrane (Hille B., 2001). We know that a single synapse consists of presynaptic and postsynaptic parts, which are further made up by tiny and intricately packed vesicles, release machineries, postsynaptic receptors and scaffolding proteins. We can even find highly detailed schematic diagrams that depict the roles of individual synaptic proteins in synaptic functions (Cowan W.M. et al., 2003).

Despite these successes, some fundamental questions concerning brain functions remained unanswered. Although we understand relatively well about the behavior of single neurons or single synaptic connections, we are still far away from 
explaining the brain's behavior given the function of these basic components. Whereas simple models exist that link the microscopic motions of molecules to the macroscopic thermodynamic properties of gases, it is unclear how we could understand the brain's behaviors in terms of the properties of individual neurons. How billions of neurons interconnected in a specific manner generate a functional network that allows us to see, to feel, to think, and to decide? How trillions of synapses following certain rules of dynamics and plasticity allow us to learn and to remember? These questions cannot be answered by studying individual neurons or individual synapses in isolation. They require investigations from an integral or systems point of view of the whole neuronal network.

The development of modern electronic devices might provide an example of how complex systems might be assembled from simple components. Although contemporary electronic devices like computers, cell phones, digital cameras etc are immensely complex and carry a wide variety of different functions, they are actually built by systematically connecting basic components called transistors. By interconnecting several transistors, one could build simple logical circuits that carry out basic operations such as NAND, NOR, NOT. By putting together these logical gates, one could design higher level subsystems that serve as input/output interfaces, data storage subsystems, and central processing units. Combining these processing units finally constitute a complete digital system. Is the brain built in a similar, hierarchical way? If so, what are the underlying rules and principles? Can we identify basic "modules" or "circuit motifs" in the brain that carry out functions as specific as a NAND or NOR gate? Can we explain the brain function as a whole by putting together these basic functional modules?

To understand these systems level questions, neuroscientists need certain 
circuit analysis toolkits. The problems faced by systems neuroscientists are like those faced by an engineer who is trying to understand programs or circuits designed by other people. A typical engineer solves these problems by introducing "break points" in the programs or by inserting "test probes" into certain nodes of the circuits. By checking the behavior of the circuitry at these test points, an engineer can gain some insights into the operation of an unknown design. Exactly the same approaches were taken by early neuroscientists as they poke microelectrodes into the brain and listen to the firing of individual neurons. These pioneering studies provided initial insights into the function of different brain areas and the information they encode. However, to understand the detail operation of a complete network, one clearly needs more powerful tools.

Substantial technical advances in recent years have provided neuroscientists with powerful tools that can hardly be imagined 10 years ago. For example, micro-fabrication technologies have enabled the construction of microelectrode arrays that can be implanted into living animals and can record the activity simultaneously from many neurons while the animals perform behavioral tasks (Buzsaki, 2004). Furthermore, modern microscopy together with fluorescent reporters for neuronal activity allows a highly detailed mapping of individual neurons' function in many brain areas (Ohki et al., 2005; Yaksi et al., 2007;Sato et al., 2007). These technologies allow reading out the brain's activity on a large scale. A major challenge now is to gain insight into the operation of the brain by interpreting these complex multi-neuronal activity data.

In this thesis, I analyzed the function of olfactory bulb (OB) neuronal networks using multi-neuronal $\left[\mathrm{Ca}^{2+}\right]$ imaging as the primary experimental tool. In this technique, a large population of neurons is stained using fluorescent dyes that 
change their fluorescent properties in response to changes in intracellular $\left[\mathrm{Ca}^{2+}\right]$ (Grynkiewicz et al., 1985). Because the electrical discharges of neurons are often accompanied by $\left[\mathrm{Ca}^{2+}\right]$ influx via voltage gated $\left[\mathrm{Ca}^{2+}\right]$ channels (Hille B., 2001), changes in intracellular $\left[\mathrm{Ca}^{2+}\right]$ can often be used as good indications for neurons' electrical firing patterns (Cossart et al., 2003; Yaksi and Friedrich, 2006;Lin et al., 2007). I focus my analysis mainly on extracting functional information from the obtained multi-neuronal activity patterns. After a brief overview of OB's circuitry, I presented a number of computational algorithms that facilitate visualizing and analyzing $\left[\mathrm{Ca}^{2+}\right]$ imaging data (Sec. 3.1). I then analyzed the spatiotemporal structure of spontaneous multi-neuronal $\left[\mathrm{Ca}^{2+}\right]$ activity patterns of OB neurons (Sec. 3.2). I then addressed how the neuronal activity patterns could be understood in terms of the underlying circuitry of the OB (Sec. 3.3). Finally, we presented data concerning the principle of odor coding by OB's neuronal circuitry (Sec. 3.4) as well as possible synaptic mechanisms underlying a coordinated firing of OB neurons (Sec. 3.5). These results will contribute to a better understanding the $\mathrm{OB}$ as an intricately connected neuronal network. 


\subsection{The olfactory bulb (OB)}

The olfactory bulb is a structure in the vertebrate forebrain involved in the perception of odors. It is the primary brain region that receives direct axonal inputs from olfactory sensory neurons (OSN) in the nose. The principal neurons of the OB, the mitral/tufted (M/T) cells, form synapses with OSN axons and send the output information of the $\mathrm{OB}$ to a number of higher brain regions. These higher centers include the anterior olfactory nucleus, the olfactory tubercle, the amygdala, the piriform cortex, and the entorhinal cortex (Greer and Shepherd, 1998;Mori et al., 1999; Lledo et al., 2005). The OB is therefore an interface between the OSN and higher olfactory centers. Its position in olfaction is often compared to the thalamus in other sensory systems (Murakami et al., 2005; Shepherd, 2005).

The anatomy of the OB circuitry has been extensively analyzed since the early works of Ramon Cajal (Cajal, 1899, Fig. 1-1). The surface of the OB contains many spherical neuropil structures called glomeruli where axons of OSNs terminate and synapse with the dendrites of M/T cells. The number of glomeruli ranged from $\sim 10^{3}$ in the mammalian OB (Lledo et al., 2005), $\sim 10^{2}$ in the OB of amphibians and fish (Nezlin and Schild, 2000; Friedrich and Laurent, 2001), and 50 in the antenna lobe of insects (analog of the vertebrate OB, Komiyama and Luo, 2006). In mammals, an individual glomerulus receives convergent axonal inputs from $\sim 10^{4}$ OSNs and is innervated by the dendrites of $\sim 10 \mathrm{M} / \mathrm{T}$ cells (Lledo et al., 2005). The OSNs that express one type of olfactory receptor are thought to project mainly to one or two glomeruli (Fig. 1-2). Such "grouping" of the dendritic and the axonal terminals of multiple neurons into distinct and segregated glomerular structures has let many investigators to consider the olfactory glomeruli as an example of "modular architectures" in the brain (Greer and Shepherd, 1998;Mori et al., 1999). In this 
respect, the olfactory glomeruli are often compared to the "columns" or "barrels" in the cerebral cortex (Greer and Shepherd, 1998).

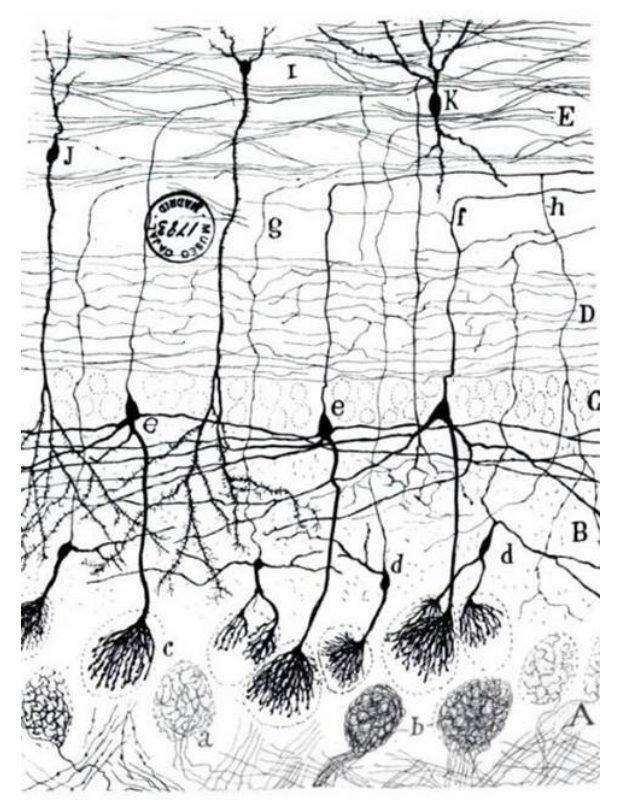

Fig. 1-1 Section of the olfactory bulb of a several-days-old kitten. A, glomerular layer; B, outer plexiform layer; C, mitral cell layer; D, innerplexiform layer; E, granule cell layer. Image taken from (Cajal, 1899)

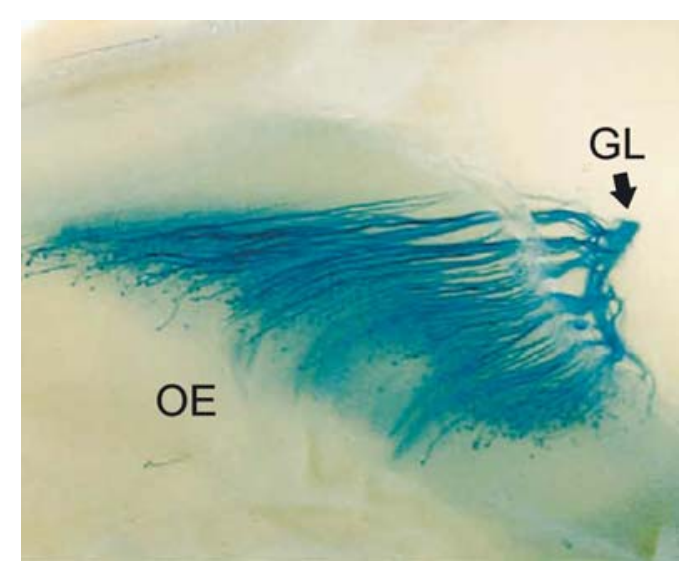

Fig. 1-2 Projecting patterns of OSNs that express the olfactory receptor gene P2. The axons of the OR specific OSNs converge on a single glomerulus on the medial aspect of the bulb. OE: olfactory epithelium, GL: glomerulus. Image taken from (Mombaerts et al., 1996) 
In addition to the principal neurons (M/T cells), the OB contains many other cell types, in particular the inhibitory interneurons (Greer and Shepherd, 1998). There are two main types of inhibitory interneurons in the OB: periglomerular cell and granule cells (Fig. 1-3). The cell bodies of periglomerular cells are located near individual glomeruli. The granule cells are located deep in the OB and form a separate cell layer, the granule cell layer. Granule cells are $\sim 50-100$ times more numerous than M/T cells (Greer and Shepherd, 1998). The large number of inhibitory neurons provides $\mathrm{M} / \mathrm{T}$ cells with strong inhibitory modulations. These inhibitory interactions play an important role in generating oscillatory network dynamics and are thought to be important for enhancing the contrast between the representations of different odors (Mori et al., 1999;Laurent, 2002;Lledo et al., 2005).

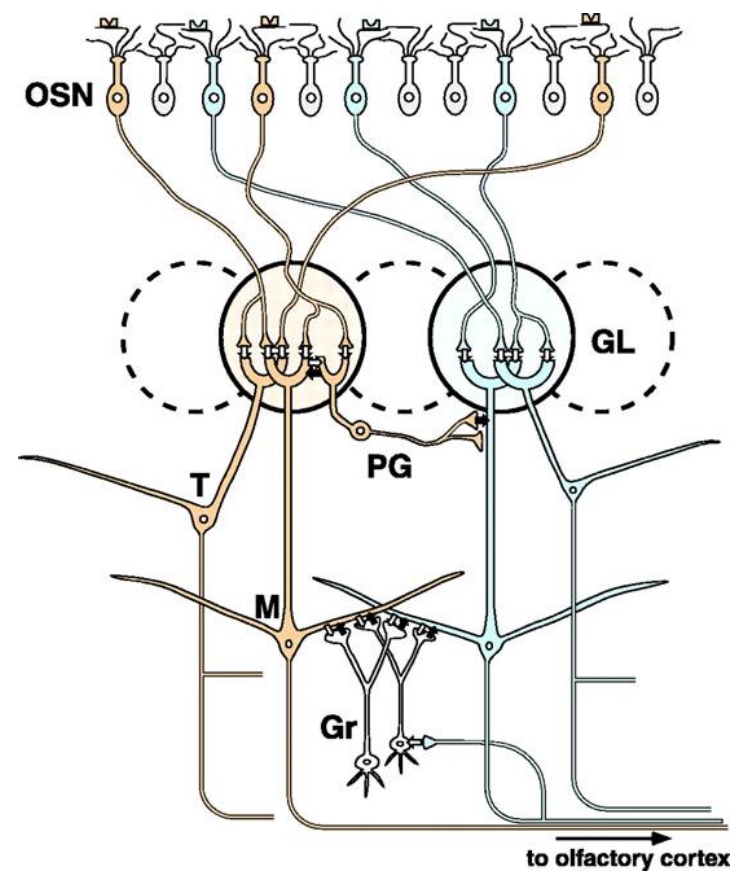

Fig. 1-3 Schematic circuit diagram summarizing the circuit organization of the olfactory bulb. Mitral cells (M) and tufted cells (T) are output neurons, and granule cells (Gr) and periglomerular cells (PG) are local interneurons. OSN, olfactory sensory neuron; GL, glomerulus. (taken from Mori et al., 1999). 


\section{Materials and Methods}

\subsection{Xenopus nose-brain preparation.}

Xenopus laevis tadpoles (stage 53-55; Nieuwkoop and Faber J., 1967) were immobilized by incubation in a mixture of ice and water. A block of tissue containing the olfactory mucosae, intact olfactory nerves, and most of the brain was cut out and kept in physiological saline (in $\mathrm{mM}, \mathrm{NaCl} 98, \mathrm{KCl} 2, \mathrm{CaCl}_{2} 1, \mathrm{MgCl}_{2}$ 2, glucose 5, Na-pyruvate 5, HEPES 10; 230 mOsm, pH 7.8). The dorsal part of the olfactory bulb was sliced off using a vibrotome (Leica VT1000S) to expose neurons for imaging and patch-clamp recordings. The olfactory mucosae and nerves were kept intact unless stated otherwise.

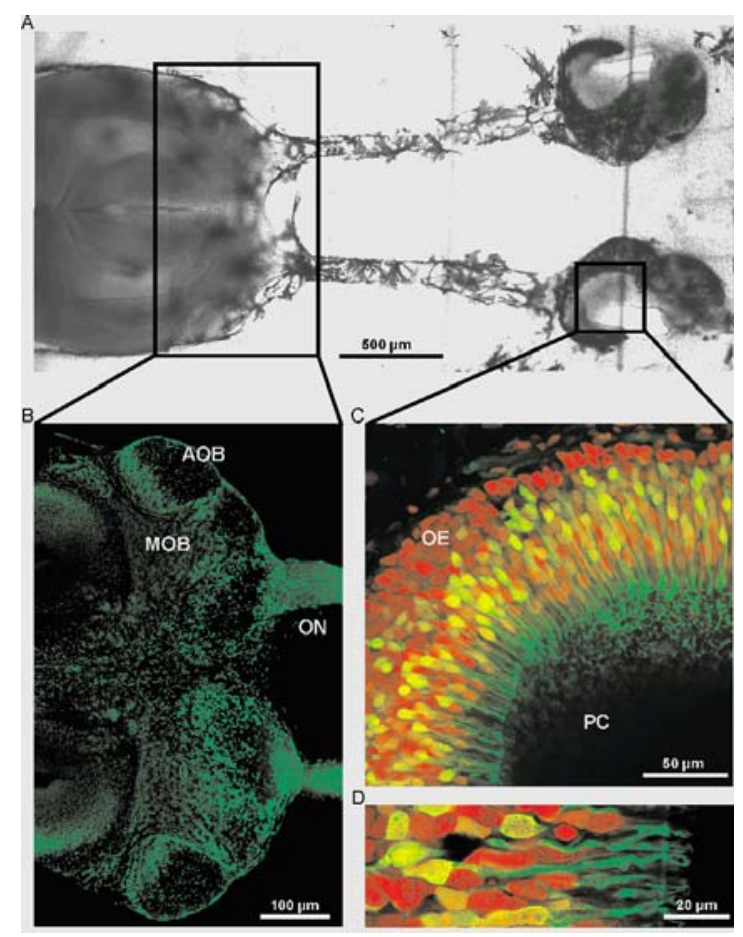

Fig. 2-1 Xenopus nose-brain prepration A, image of the slice of the olfactory mucosa and the olfactory bulb. $B$, slice of the anterior part of the brain including the olfactory nerve $(\mathrm{ON})$, the main olfactory bulb (MOB) and the accessory olfactory bulb (AOB) stained with propidium iodide. $C$, horizontal overview of the olfactory epithelium (PC, principal cavity and OE, olfactory epithelium). $D$, higher magnification of $C$ (taken from Manzini et al., 2002) 


\subsection{Dye loading and $\left[\mathrm{Ca}^{2+}\right]$ imaging.}

To load neurons with $\left[\mathrm{Ca}^{2+}\right]$ sensitive dye, the preparations were incubated in a bath solution containing $50 \mu \mathrm{M}$ of Fura-2/AM or Fluo-4/AM (Molecular Probes, Eugene, OR) for 30 minutes at room temperature, and then rinsed with bath solution for at least 30 minutes in the recording setup. For experiments involving imaging neurons located below slice surface (e.g. Fig. 3-22), a pressure injection protocol was used to load neuronal populations with $\left[\mathrm{Ca}^{2+}\right]$ sensitive dye (Stosiek et al., 2003). In this approach, Fluo-4/AM was first dissolved in 20\% Pluronic F-127 in DMSO, and then diluted in the bath solution to reach a final concentration of $500 \mu \mathrm{M}$. This solution was then injected at a depth of around $70 \mu \mathrm{m}$ using patch pipettes with a series resistance of $6-8 \mathrm{M} \Omega$ at a pressure of $\sim 100 \mathrm{hPa}$ for $5 \mathrm{~min}$ in one-three sites per bulb. The progress of the injection was monitored using a 40x water immersion objective. The activity of the neurons was measured after $\sim 30$ min following the last injection.

$\left[\mathrm{Ca}^{2+}\right]$ measurements with Fura-2 were performed using an upright microscope (Axioskop 2, Zeiss, Göttingen, Germany) with a 40X $(\mathrm{NA}=0.9)$ objective.

Fluorescence images excited at $380 \mathrm{~nm}$ were taken at $5 \mathrm{~Hz}$ using a frame-transfer, back-illuminated CCD camera (Micromax, Visitron, Munich, Germany). Confocal imaging with Fluo-4/AM was performed using a laser scanning microscope (Zeiss LSM 510/Axiovert 100M). The following objectives were used: Achroplan 40x/0.8 W; Plan-NeoFluar 10x/0.3; Plan-Apochromat 40x/1.3 oil (Zeiss, Göttingen, Germany). 


\subsection{Odorant stimulation}

A mixture of 14 L-amino acids (alanine, serine, threonine, cysteine, valine, leucine, isoleucine, methionine, proline, arginine, lysine, histidine, phenylalanine, and trytophan, $10 \mu \mathrm{M}$ in Ringer's solution) or single amino acids (50-100 $\mu \mathrm{M}$ in Ringer's solution) were used as odorants. Short pulses (1s) of odorant were applied by triggering odor delivery valves (LFAA1200218H, The LEE CO) using custom build recording software.

To avoid possible effects of adaptation, consecutive odor stimulations were separated by at least 50s. In experiments involving many odors, repeated stimulations of an odor were interleaved with the application of other odorants.

\subsection{Electrophysiology and morphological reconstructions.}

Imaging data were first analyzed online to select neurons showing synchronous activity. The same cells were then re-identified under transmission optics and patch pipettes containing internal solution (in $\mathrm{mM}, \mathrm{NaCl} 2, \mathrm{KCl} 11, \mathrm{MgSO}_{4} 2$, K-gluconate 80, HEPES 10, EGTA 0.2, ATP 3, GTP 0.3) were directed to the selected neurons under visual control. In some recordings (Fig. 3-29 and Fig. 3-30), a $\mathrm{Cs}^{+}$-based internal solution (in $\mathrm{mM}, \mathrm{NaCl} 1, \mathrm{MgCl}_{2}$ 1, TEA-Cl 11, $\mathrm{CsCH}_{3} \mathrm{SO}_{4} 79$, HEPES 10, EGTA 5, ATP 3, GTP 0.3) was used. For morphological reconstructions, $200 \mu \mathrm{M}$ Alexa-488 or Alexa-555 (Molecular Probes, Eugene, OR) were added to the recording pipette solution. To avoid any ambiguity, only two cells (one color each) were subjected to staining attempts in each bulb. The slices were fixed in $4 \%$ paraformaldehyde (Sigma) for 2 hours and rinsed. The bulbs were then excised and mounted in $85 \%$ glycerol. The cells' morphology was reconstructed by taking confocal 3D stacks. 


\subsection{Data analysis}

Image analysis was performed using custom programs written in MATLAB (MathWorks, Natick, MA). Regions of interest (ROI) corresponding to cell somata were selected using a semi-automatic algorithm and the average fluorescence intensities of individual ROIs were measured as a function of time. The background of each ROI was determined using a pixel-based algorithm (Chen et al., 2006) and subtracted prior to the calculation of $\Delta \mathrm{F} / \mathrm{F}$ values.

To identify synchronous M/T cells, fluorescence images were recorded over $180 \mathrm{~s}$ in the absence of stimulation. Slow baseline drifts of every trace (calculated by smoothing the trace with a Hanning filter, window length $8 \mathrm{~s}$ ) were first subtracted from each trace (Cossart et al., 2003). The activity correlation index was defined as the zero-lag cross-correlation value between pairs of baseline-subtracted traces.

Neighborhood correlation maps (NCM) or cross-correlation maps (CCM) were generated using custom programs written in Matlab (The MathWorks, USA). A bleach correction was first performed for all pixels of the images by subtracting a linear trend from each pixel's time trace. In NCM, a "neighborhood signal" $n(t)$ was first generated by averaging the fluorescence signals of four pixels immediately surrounding a given pixel. The pixel's value in the NCM was then generated by calculating the correlation coefficient between the pixel's signal $f(t)$ and the neighborhood signal $n(t)$. In CCM, a reference trace $r(t)$ was obtained by averaging the fluorescence intensities across a given region of interests (ROI) for each time point. The pixel's value in the CCM was then generated by calculating the correlation coefficient between the pixel's signal $f(t)$ and the reference signal $r(t)$. For experiments where $\left[\mathrm{Ca}^{2+}\right]$ signals were sampled at a higher rate $(5-10 \mathrm{~Hz}$ in epi-fluorescence imaging), a slow baseline drift (calculated by smoothing the trace 
with a Hanning filter, window length $8 \mathrm{~s}$ ) was subtracted from the reference trace before calculating the correlation maps. This procedure emphasizes the rising phases of the $\left[\mathrm{Ca}^{2+}\right]$ signals and is effective in reducing un-specific correlations caused by the slow decays of $\left[\mathrm{Ca}^{2+}\right]$ signals.

Data values were reported as mean \pm s.e.m. unless otherwise noted. 


\section{Results}

This thesis focuses on a systems level analysis of the coding function of olfactory bulb neuronal circuit, using $\left[\mathrm{Ca}^{2+}\right]$ imaging as the primary experimental technique. I start the results section by introducing a number of image analysis tools that facilitate the visualization of signals in population $\left[\mathrm{Ca}^{2+}\right]$ imaging data (Sec. 3.1). In the remaining sections (Sec. 3.2-3.5), I describe several findings concerning the coding and the modular organization of OB neurons.

\subsection{Algorithms for visualizing $\left[\mathrm{Ca}^{2+}\right]$ imaging data}

\subsubsection{Neighborhood Correlation Map}

One of the major goals in fluorescence $\left[\mathrm{Ca}^{2+}\right]$ imaging is to capture how the $\left[\mathrm{Ca}^{2+}\right]$ signals of the imaged structures change over time. Thus, it is often desirable to know where in the image a change in fluorescence intensity occurs. One obvious way to do so would be simply playing the recorded data as a movie and then manually marking the structures that show certain intensity change during the recording period. However, for a long recording that contains many neurons, this could be a painful process. Thus, it is desirable to have a single map that highlights the locations of pixels whose fluorescence intensity exhibits changes during the recording period.

A major requirement of such a map is that it should distinguish between the "meaningful" intensity changes due to $\left[\mathrm{Ca}^{2+}\right]$ signals and stochastic changes caused by random noise. Because photon detection is intrinsically a probabilistic process, the signals detected at individual pixels will always show some fluctuations even when the underlying intensity did not exhibit any change. It is important that the detection of these random fluctuations is minimized in this map. I propose one approach that satisfies the above requirement: the Neighborhood Correlation Map (NCM) (Fig. 3-1). 
In this map, the brightness of an individual pixel encodes the degree of correlation between the pixel's signal and the signals of other pixels located immediately adjacent to the pixel (Fig. 3-1). Thus, a pixel that appears bright in the NCM shows a high correlation to its neighboring pixels whereas a pixel that appears dark shows little or no correlation to its nearby pixels.

In high resolution imaging, an imaged structure (such as a soma or a dendrite of a cell) is often larger than the size of a single pixel. Thus, any change in fluoresce intensity of this structure will be detected by multiple pixels, and these pixels will therefore exhibit a rather similar temporal signal waveform. This is illustrated by cell 1 in Fig. 3-1. This neuron exhibits two odorant-induced $\left[\mathrm{Ca}^{2+}\right]$ transients that are detected by all pixels belonging to the cell. Thus, adjacent pixels of the same cell carry correlated signals reflecting this common process, which leads to a bright labeling of this neuron in the NCM. In contrast, cell 2 did not show any "meaningful" change in fluorescence intensity. Thus, pixels belonging to this cell show random fluctuations in fluorescence intensity reflecting photon noise. Because these noises are generally uncorrelated, pixels belonging to this cell show low correlation of their fluorescence signals. This leads to a dark labeling of this neuron in the NCM, even though it appears very bright in the original image. In this way, the NCM allows for specifically highlighting neurons that show activity during the recording period. 


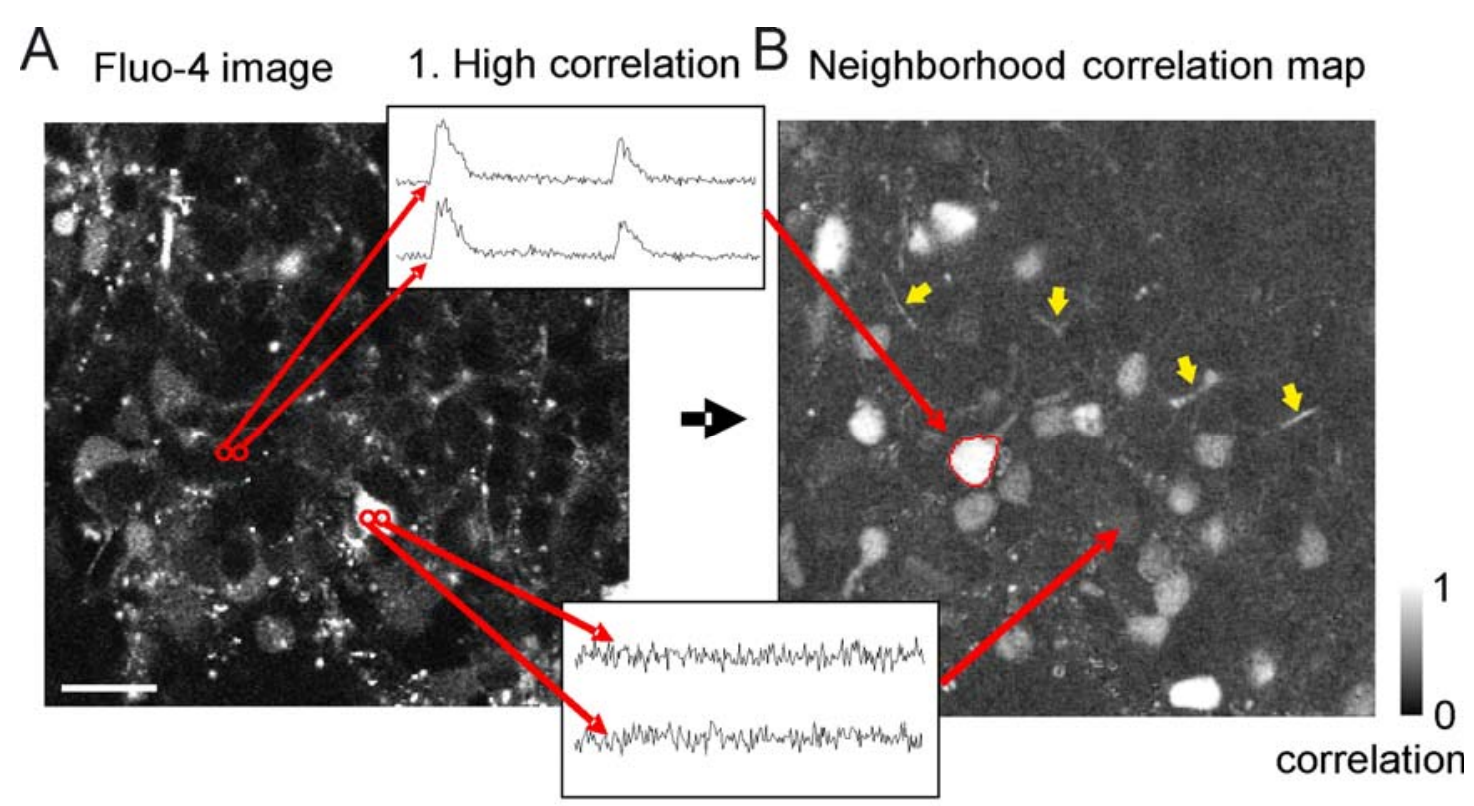

2. No correlation

Fig. 3-1 $\left[\mathrm{Ca}^{2+}\right]$ imaging of odor responses in population of $\mathrm{OB}$ neurons stained with Fluo-4. (A) Raw Fluo-4 image, most neurons cannot be clearly seen. (B) A Neighborhood Correlation Map (NCM) in which the brightness of individual pixels encodes the degree of correlation between the pixel's signal and the signals of nearby pixels. Neurons showing activity during the recording period (e.g. Cell 1, top) are clearly labeled in this map. In contrast, neurons showing no activity (e.g. Cell 2, bottom) disappear in the NCM even though they might appear bright in the Fluo-4 image. Yellow arrows: some active dendritic segments can also be seen. Scale bar: 20 $\mu \mathrm{m}$.

The ability of NCM to visualize active neurons is particularly useful when the raw fluorescence image does not allow a clear morphological distinction of neurons. This is often the case when using Fluo-4 (and some other dyes) as $\left[\mathrm{Ca}^{2+}\right]$ indicator because this dye typically shows a very low fluorescence at a low $\left[\mathrm{Ca}^{2+}\right]$ level. As the resting $\left[\mathrm{Ca}^{2+}\right]$ of healthy neurons is typically low, these neurons are often hardly visible in the raw Fluo-4 images (Fig. 3-1, left). NCM gives a way to clearly localize and visualize active neurons while at the same time removing brightly labeled structures (e.g. dead neurons with high $\left[\mathrm{Ca}^{2+}\right]_{\mathrm{i}}$ ) that do not exhibit any activity. 
The ability of NCM to visualize active neurons makes it particularly suitable for guiding the selection of regions of interest (ROIs). In the example shown in Fig. 3-2, NCM helps to identify and to define contours of active neurons that are not clearly visible in the Fluo-4 image. Measuring $\left[\mathrm{Ca}^{2+}\right]$ signals from these neurons allows me to build an ensemble representation of odors by a local population of OB neurons (Fig. 3-2, right).
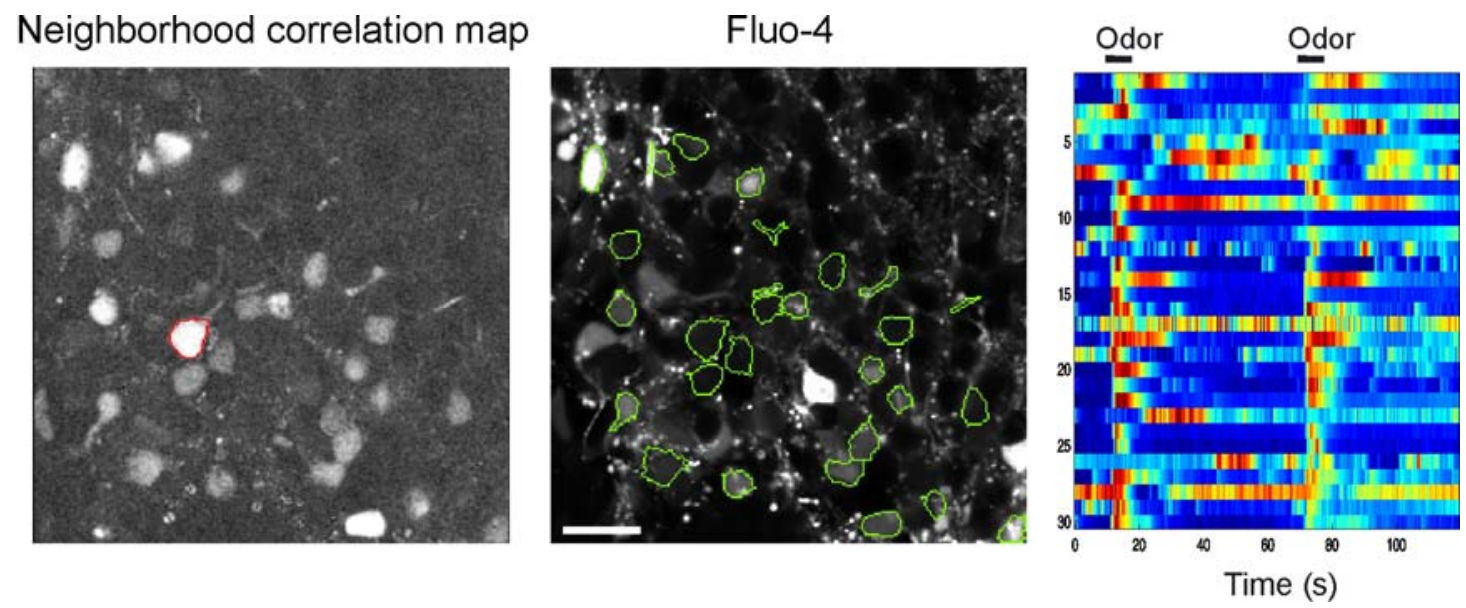

Fig. 3-2 Neighborhood Correlation map guides the selection of regions of interest A clear labeling of active neurons in the neighborhood correlation map (left) allows defining contours of active neurons and dendrites (middle) and measuring signals from those structures (right). Scale bar: $20 \mu \mathrm{m}$.

In addition to visualizing active somata, NCM also can be used to visualize dendritic compartments that exhibit fluorescence dynamics. In the example shown in Fig. 3-1, the NCM labels various dendritic segments (yellow arrows) in additional to the many labeled somata. This enables selecting ROIs and measuring signals from dendrites that are not visible in the raw Fluo-4 images (Fig. 3-2). The ability of NCM to visualize neuropile structures is further utilized by Drs. Ivan Manzini and Christoph Brase in our lab to obtain a high resolution map of response distribution within individual olfactory glomeruli (Fig. 3-3). In these experiments, they measured the 
$\left[\mathrm{Ca}^{2+}\right]$-dependent fluorescence change in the glomerular layer of the olfactory bulb during stimulations with odors. Whereas the structure of a glomerulus can not be clearly seen in the Fluo-4 image both before and at the peak of odor-responses, the NCM reveals striking structural details of a glomerulus during odor stimulation (Fig. 3-3).
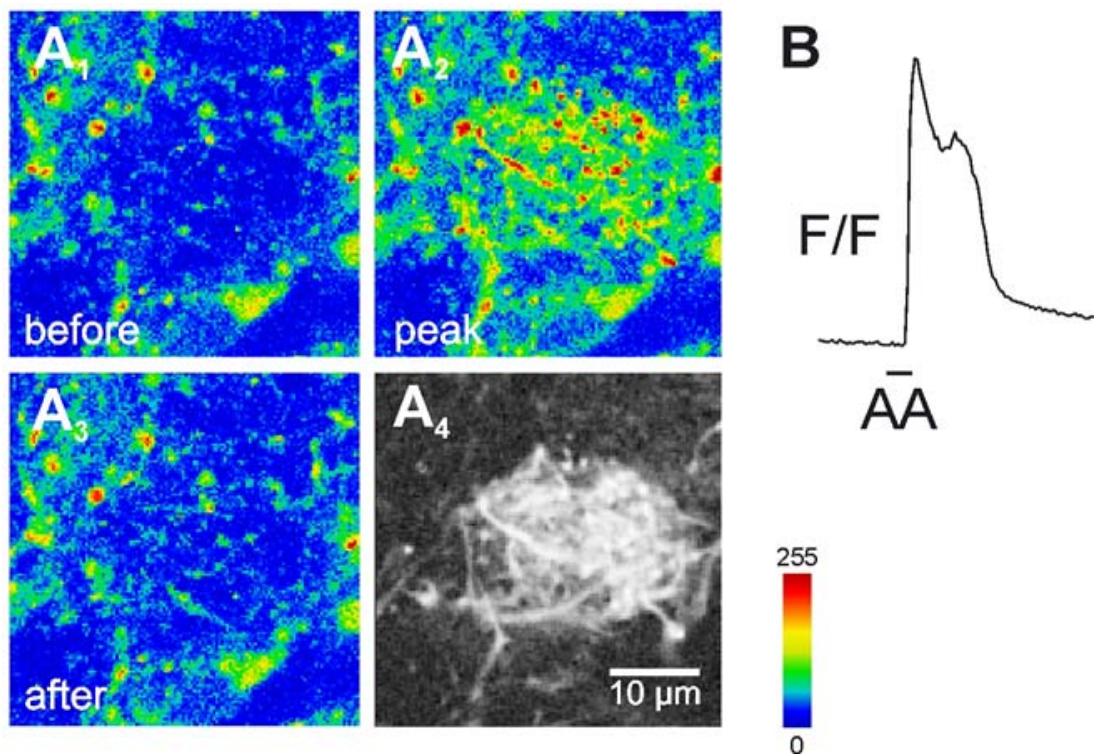

Fig. 3-3 Visualizing the responses within an individual glomerulus using Neighborhood Correlation Map $\left[\mathrm{Ca}^{2+}\right]$ imaging of odor responses in the glomerular layer of the olfactory bulb. The color coded images (A1-A3) shows the Fluo-4 image before, at the peak, and after odor responses. The gray scale image (A4) shows the Neighborhood Correlation Map calculated from the same data. The fine dendritic structures of the glomerular neuropile are clearly visible. Right, the $\left[\mathrm{Ca}^{2+}\right]$ response waveform measured in the glomerulus (taken from Manzini et al., 2007) 


\subsubsection{Cross-Correlation Map}

NCM labels all structures in the image that exhibit some "meaningful" changes in fluorescence intensity. However, in many cases, one might be interested in knowing which structures exhibit a specific signal waveform. For this purpose, I introduced a second technique: the Cross-Correlation Map (CCM). In this map, the brightness of an individual pixel encodes the cross-correlation value between the pixel's signal and a certain reference waveform. In this way, CCM allows visualizing the parts of an image that exhibit signals similar (correlated) to the reference waveform.

In fact, the concept of using cross-correlation as a contrast variable to visualize the distribution of signals is not new. In functional magnetic resonance imaging (fMRI), the so-called "activation map" is actually a map of correlation values between each voxel's BOLD signal and a "reference waveform" that reflects the timings of external stimulations (Frahm, 1993). This allows mapping the parts of the brain whose blood flow signals show a significant correlation to the external stimulus events. However, despite its extensive use in fMRI, cross-correlation has not been employed, to my knowledge, as a contrast variable for visualizing the distribution of signals in sequences of $\left[\mathrm{Ca}^{2+}\right]$-dependent fluorescence images.

Fig. 3-4 shows an example of CCM generated using the same data set as in Fig. 3-1. The map is calculated using the $\left[\mathrm{Ca}^{2+}\right]$ signal of an odor-responsive neuron (arrow) as the reference waveform. The correlation map is then thresholded in a way similar to what is typically used in fMRI to reveal significantly correlated pixels (Frahm, 1993). This thresholded map is then overlaid on the Fluo-4 image. This map labels the pixels whose signals show significant correlation to the reference waveform. That is, it 
specifically labels the cells that show excitatory responses to odorant stimulation.
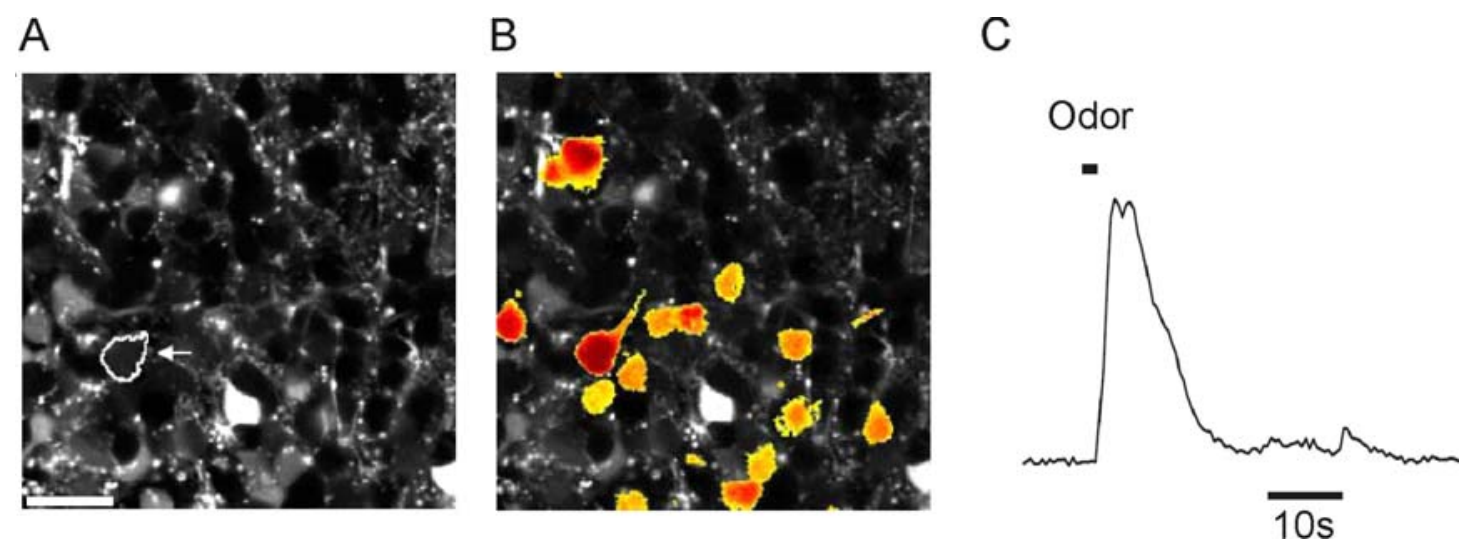

Fig. 3-4 Cross-Correlation Map labels responsive neurons in the OB. (A) $\left[\mathrm{Ca}^{2+}\right]$ imaging of odor responses in OB neurons, same data as in Fig. 3-1. (B) Cross-Correlation Map constructed using the odor response waveform of a selected neuron (arrow in A) as the reference waveform. The CCM is thresholded and overlaid on the raw Fluo-4 image. This labels the location of neurons that show similar response waveforms to the marked neuron. (C) The odor response waveform of the selected neuron as the reference waveform. Scale bar: $20 \mu \mathrm{m}$.

As in the NCM, the CCM can reveal structures other than a neuron's soma. This is illustrated by the example shown in Fig. 3-5. In this example, I measured $\left[\mathrm{Ca}^{2+}\right]$ signals from neurons in the $\mathrm{M} / \mathrm{T}$ cell layer. These neurons are known to show a rich pattern of spontaneous activity even in the absence of any stimulation. I took the spontaneous $\left[\mathrm{Ca}^{2+}\right]$ activity of a neuron and calculated the corresponding CCM. This map not only showed the soma of the neuron but also revealed three processes that were not visible in the original Fura-2 image. The CCM further guided me to select ROIs and measure signals from these processes. Indeed, measuring from these regions yielded signals highly similar to the somatic signals. This further suggests that the processes revealed in the CCM are indeed the processes of the selected neuron.

The calculation of CCM can be done independently for individual neurons using their $\left[\mathrm{Ca}^{2+}\right]$ signals as reference waveforms. In Fig. 3-6, I calculated four 
correlation maps using the $\left[\mathrm{Ca}^{2+}\right]$ signals of four different neurons as reference waveforms. Each of these maps revealed some dendrites of the respective neurons that were not visible in the raw Fura-2 image. By coding individual maps using different colors, we could visualize neurons with a markedly enhanced contrast, as if individual neurons were "stained" using dyes of different colors. This map also provided a highly condensed way to visualize the information contained in the original fluorescence image sequence. Within a single representation, the spatial distribution of different signal waveforms can be visualized, each using a different color.

A

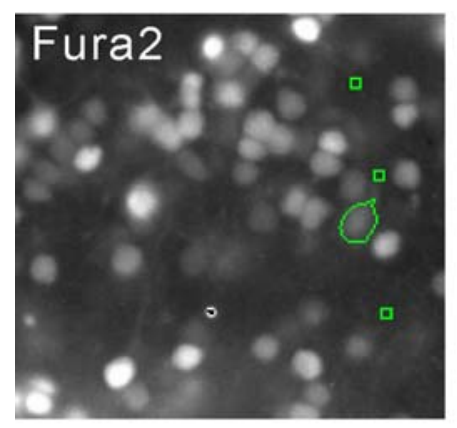

B

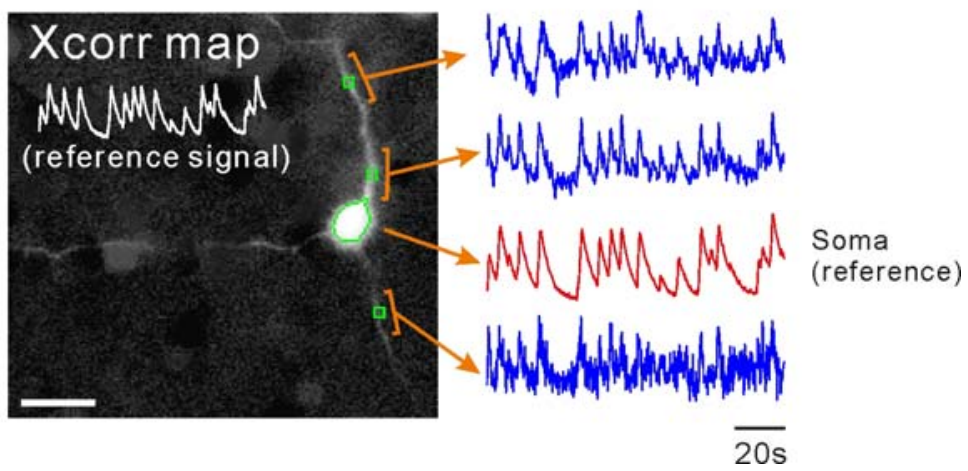

Fig. 3-5 Visualizing dendritic processes in the Cross-Correlation Map (A) $\left[\mathrm{Ca}^{2+}\right]$ imaging of spontaneous activity of mitral/tufted cells stained using Fura-2. The processes of the neurons are hardly visible due to a lack of contrast. (B) Cross-Correlation Map calculated with respect to the signal of the marked soma (reference signal). In this map, the brightness of individual pixels encodes the degree of cross-correlation between the pixels' fluorescence signals and the reference signal. Three processes of the cell can be visualized due to their correlated $\left[\mathrm{Ca}^{2+}\right]$ signals (right). Only one neuron is labeled (in contrast to the case in Fig. 3-4) because the spontaneous activity between most neurons are uncorrelated. Scale bar: $20 \mu \mathrm{m}$. 


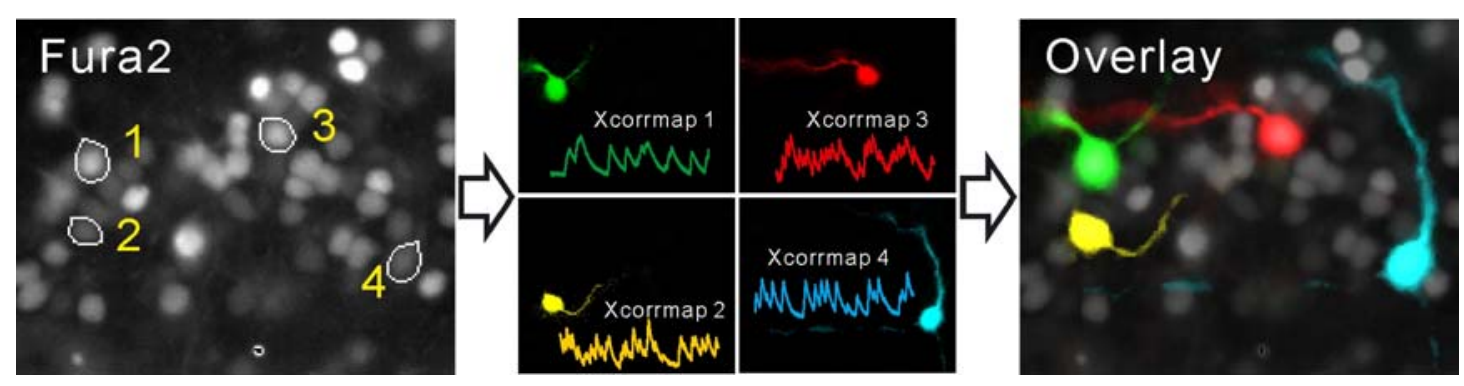

Fig. 3-6 Multi-color "labeling” of neurons using cross-correlation maps.

Cross-Correlation maps for four neurons (marked in left) coded with different colors (middle) and overlaid on the raw fluorescence image (right). The $\left[\mathrm{Ca}^{2+}\right]$ signals used to generate the maps are shown on the corresponding correlation maps (middle).

The ability of the CCM to visualize processes of particular neurons might potentially be very useful. Because neuronal processes are intricately entangled, it is often difficult to trace the processes of individual neurons in transmission images or in fluorescence images where all neurons are un-specifically stained with $\left[\mathrm{Ca}^{2+}\right]$ sensitive dyes (e.g. Fig. 3-6, left, see also Garaschuk et al., 2006;Nagayama et al., 2007). Thus, defining the connectivity of individual neurons during functional imaging of population activity remains a highly challenging task (Garaschuk et al., 2006; Nagayama et al., 2007). The "multi-color" labeling of neuronal processes using CCM as shown in Fig. 3-6 could potentially be useful to enhance the visualization of circuit connectivity during functional imaging.

One critical issue that determines the usefulness of CCM in visualizing neuronal morphology is how far one can trace a neuron's processes in the CCM. In the example show in Fig. 3-6, the CCM mostly reveal relatively short stretches of neuronal processes located relatively close to the somata. This might reflect the fact that distal processes went out of focus, or alternatively, the $\left[\mathrm{Ca}^{2+}\right]$ signal might decrease along the dendrites so that it was no longer detectable at locations far away from the soma. To address this issue, I performed experiments using an objective with a low numerical aperture $(10 \mathrm{X} / 0.3)$ and a correspondingly thicker focal depth. In 
these experiments, the CCM revealed longer stretches of neuronal processes and, in some cases, I can even trace the dendrites of mitral cells from the soma to the glomerular endings located $>150 \mu \mathrm{m}$ away from the soma. Moreover, secondary dendrites could be clearly identified (Fig. 3-7). The high contrast of the CCM can be used as a "mask" to guide the selection of ROIs on dendrites and even at distal dendritic tufts (Fig. 3-7 D). This allows to measure signals from different compartments of a neuron even though these structures are not visible in the raw fluorescence image (Fig. 3-7 A). This result shows that the CCM can be used to visualize and to guide the measurement of signals from a neuron's processes even when they are located far away from the soma.

In summary, I developed two complementary ways to visualize signals in sequences of fluorescence images. The NCM reveals structures in the image that exhibit some activity during the recording period. The CCM specifically reveals the parts of an image that exhibit a particular signal waveform. Both methods can visualize the soma and the processes of neurons and can guide the selection of ROI in structures that are hardly visible in the raw fluorescence image. In the following sections, I specifically used these tools to analyze the organization and the coding properties of OB neuronal circuits. 

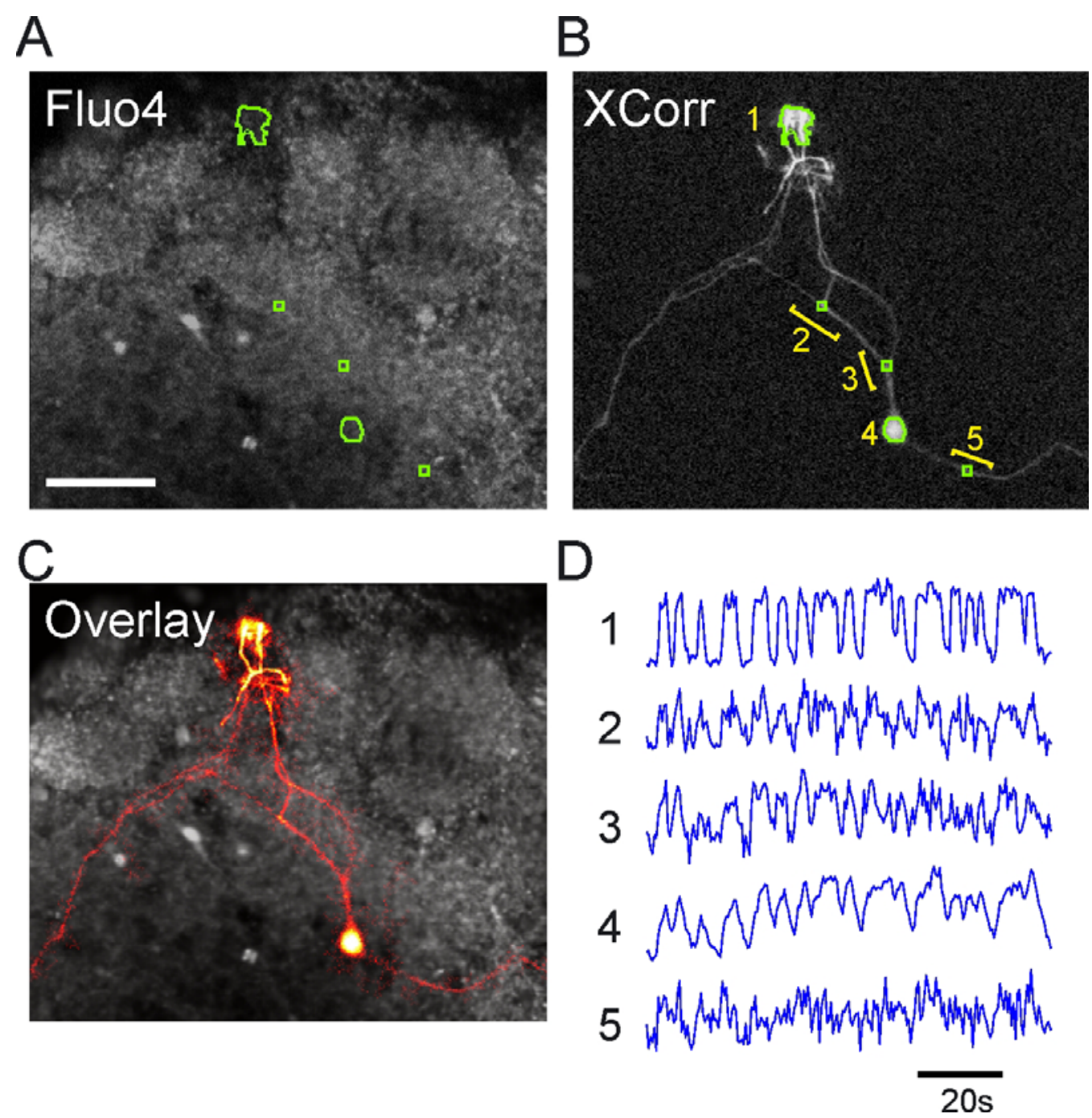

Fig. 3-7 Visualizing the glomerular connectivity of mitral cells in the cross-correlation map. (A) $\left[\mathrm{Ca}^{2+}\right]$ imaging of spontaneous activity using a low magnification objective (10X, NA=0.3). (B) The cross-correlation map calculated with respect to the signal of the selected soma. The connection of the neuron to the glomerulus can be clearly visualized. (C) Overlay of the correlation map on the Fluo-4 image. (D) The correlation map guides the measurement of signals from different compartments of the neuron even when they are not visible in the raw Fluo-4 image. Scale bar: $50 \mu \mathrm{m}$. 


\subsection{Correlation structures of OB spontaneous activity}

In this part of the thesis, I analyzed the spatiotemporal structures of the spontaneous activity in population of mitral/tufted $(\mathrm{M} / \mathrm{T})$ cells. It has been shown in a previous study of our lab that neurons in the $\mathrm{M} / \mathrm{T}$ cell layer exhibit prominent spontaneous activity in the absence of any stimulation (Lin et al., 2007). More specifically, $\sim 90 \%$ of the cells in the M/T cell layer exhibit some spontaneous $\left[\mathrm{Ca}^{2+}\right]$ transients during a three-minute period (Lin et al., 2007). These $\left[\mathrm{Ca}^{2+}\right]$ transients are TTX dependent (Chen et al., 2006; Lin et al., 2007). Moreover, the rising phases of the spontaneous $\left[\mathrm{Ca}^{2+}\right]$ transients correspond tightly to the firing of action potentials in these neurons (Lin et al., 2007) (Fig. 3-8). Thus, measuring neuronal $\left[\mathrm{Ca}^{2+}\right]$ activity provides a way to reconstruct the intrinsic firing patterns of $\mathrm{M} / \mathrm{T}$ cells.

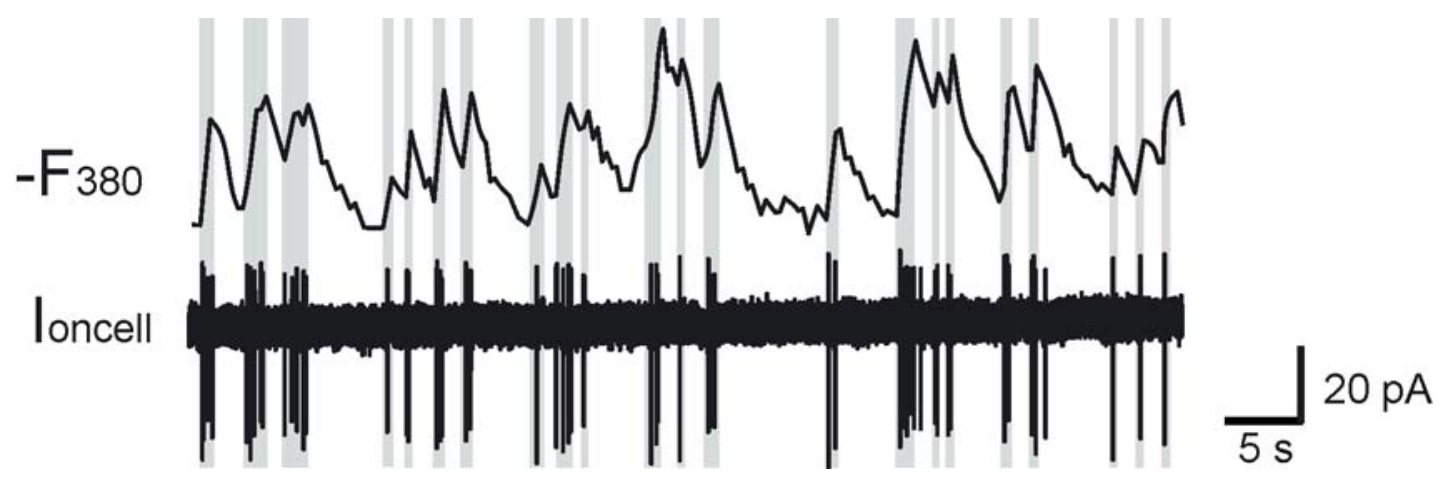

Fig. 3-8. Simultaneous recording of spontaneous $\left[\mathrm{Ca}^{2+}\right]$ transients (upper trace) and spiking (lower trace) in a mitral/tufted cell. The recording was obtained following the protocol described in (Lin et al., 2007).

Fig. 3-9 shows an example of spontaneous $\left[\mathrm{Ca}^{2+}\right]$ activity in the $\mathrm{M} / \mathrm{T}$ cell layer. The intrinsic activity patterns of these neurons were highly complex and did not show any obvious spatiotemporal structure. I did not notice any "wave" like propagation of $\left[\mathrm{Ca}^{2+}\right]$ activity across neurons. Moreover, the activity of nearby neurons appears to be 
very different. This complex pattern of activity seems to be conveying a lot of information. This motivated me to perform a more detailed investigation of these activity patterns.

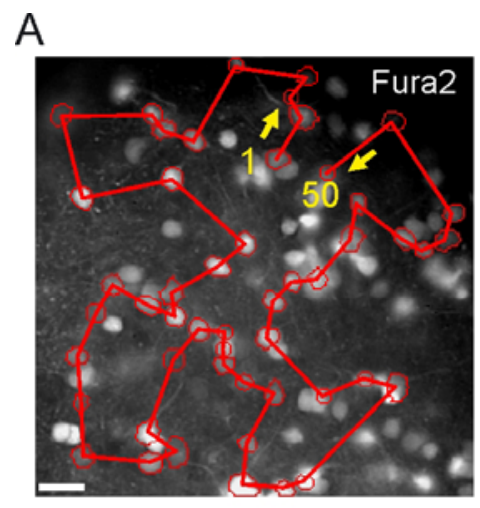

B

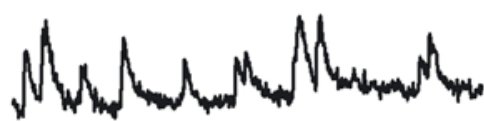

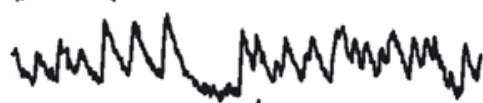

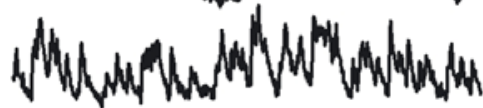

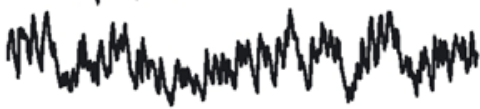

20 s

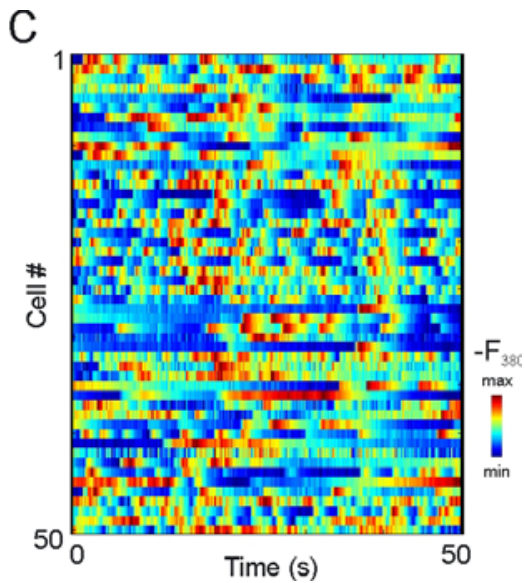

Fig. 3-9 Spontaneous activity in population of mitral-tufted cells. (A) $\left[\mathrm{Ca}^{2+}\right]$ imaging of spontaneous activity in mitral/tufted cells stained with Fura-2. Fifty spontaneously active neurons are selected. These neurons are numbered from 1 to 50 along a minimal distance path (red). (B) Spontaneous $\left[\mathrm{Ca}^{2+}\right]$ signals of four representative cells. (C) Spontaneous activity of the 50 neurons each shown as a color coded bar. The numbering of the cells follows the scheme in A. Nearby neurons (i.e. consecutive rows in this map) did not show a clear correlation. Scale bar: $20 \mu \mathrm{m}$.

\subsubsection{Identification of synchronous neuronal modules}

As a first step to approach the activity patterns, I analyzed whether there are some correlations among neurons. For this purpose, I used the cross-correlation map (CCM) introduced in the previous section to visualize the spatial locations of pixels that are correlated to the $\left[\mathrm{Ca}^{2+}\right]$ signal of a particular cell. The CCM mostly showed a brightly labeled soma (and sometimes some processes) corresponding to the selected cell (Fig. 3-5). The "background" labeling was generally very low, suggesting that the spontaneous $\left[\mathrm{Ca}^{2+}\right]$ activity of most cells were uncorrelated. However, in some cases, I observed a highly specific labeling of other neurons in the CCM. In the example shown in Fig. 3-10, using the $\left[\mathrm{Ca}^{2+}\right]$ signal of Cell 1 as the reference trace specifically 
labeled two other neurons in the CCM (arrows). The labeled neurons can be located some distances away from the reference cell with many uncorrelated neurons in between them. Most strikingly, measuring $\left[\mathrm{Ca}^{2+}\right]$ signals from these labeled neurons yielded traces that appear to be virtually identical to the reference traces (Fig. 3-10, right).
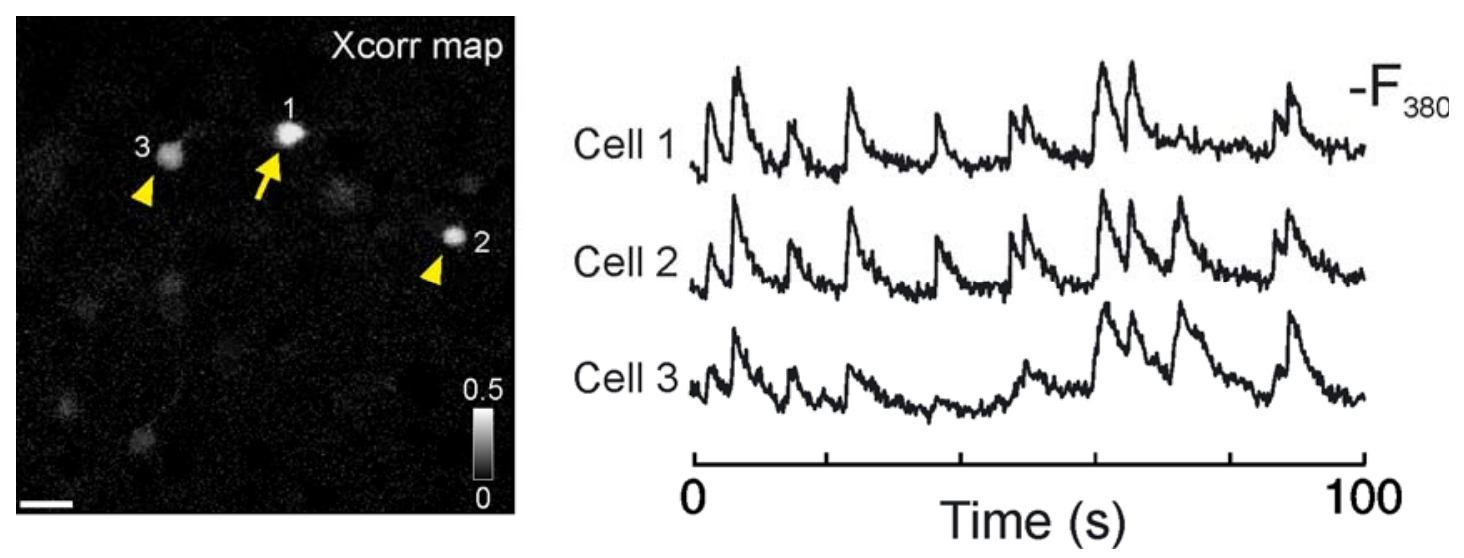

Fig. 3-10 Identification of highly correlated neurons Left, the cross-correlation map calculated with respect to cell1 labeled two other cells. Right, $\left[\mathrm{Ca}^{2+}\right]$ signals of the three correlated neurons. Scale bar: $20 \mu \mathrm{m}$.

To further analyze the correlated activity, I used a cell-based approach. I first selected all neurons in the image that show some activity during the recording period (generally 2-3 minutes) and measured their $\left[\mathrm{Ca}^{2+}\right]$ signals. The signals from $\mathrm{N}$ simultaneously recorded neurons were then used to construct an $\mathrm{NxN}$ correlation matrix in which the $\mathrm{I}^{\text {th }} \times \mathrm{J}^{\text {th }}$ elements showed the activity correlation index (r, see method) between the $\mathrm{I}^{\text {th }}$ cell and the $\mathrm{J}^{\text {th }}$ cell. Fig. 3-11 shows one example of such correlation matrix. Whereas the correlations between most cell pairs were close to zero, there were many specific subgroups of neurons that showed very high correlations $(r>0.6)$. Fig. 3-11 shows the locations of neurons belonging to different correlated groups using different colors. Neurons of the same correlated group were sparsely located and were intermingled with neurons belonging to other correlated groups. 
I performed such experiments in a total of 19 optical sections ( 9 bulbs). This allowed me to collect spontaneous $\left[\mathrm{Ca}^{2+}\right]$ signals from 1325 cells (50325 pairs). The histogram of the correlation indices clearly showed a bimodal distribution with a second peak at the higher correlation range (Fig. 3-12), indicating the presence of highly correlated neurons. There were 73 pairs of neurons that showed correlation indices larger than 0.6. The distance between these pairs ranged from 10 to $184 \mu \mathrm{m}$ (average: $53 \pm 37 \mu \mathrm{m}, \mathrm{SD} ; \mathrm{n}=73$ pairs). Fig. $3-13$ shows the histogram of the distances of the 73 highly correlated pairs. Normalizing the number of correlated pairs within a given distance bin to the total number of recorded pairs in the same bin allowed estimating the probability of finding correlated pair as a function of distance (Fig. 3-13, right). This probability decreased monotonically with distance and can be fitted by a single exponential function: $P(r)=A e^{-\frac{r}{\lambda}}$, with $A=0.0076$ and $\lambda=43.1 \mu \mathrm{m}$. I further analyzed the correlation indices of the 73 pairs as a function of the distances. The correlation indices of these pairs ranged from 0.6-0.95, and there was no significant dependency between the correlation indices of these neurons and their distances (Fig. 3-14 $\left.\mathrm{R}^{2}=0.0035, \mathrm{P}>0.64\right)$. 


\section{A}
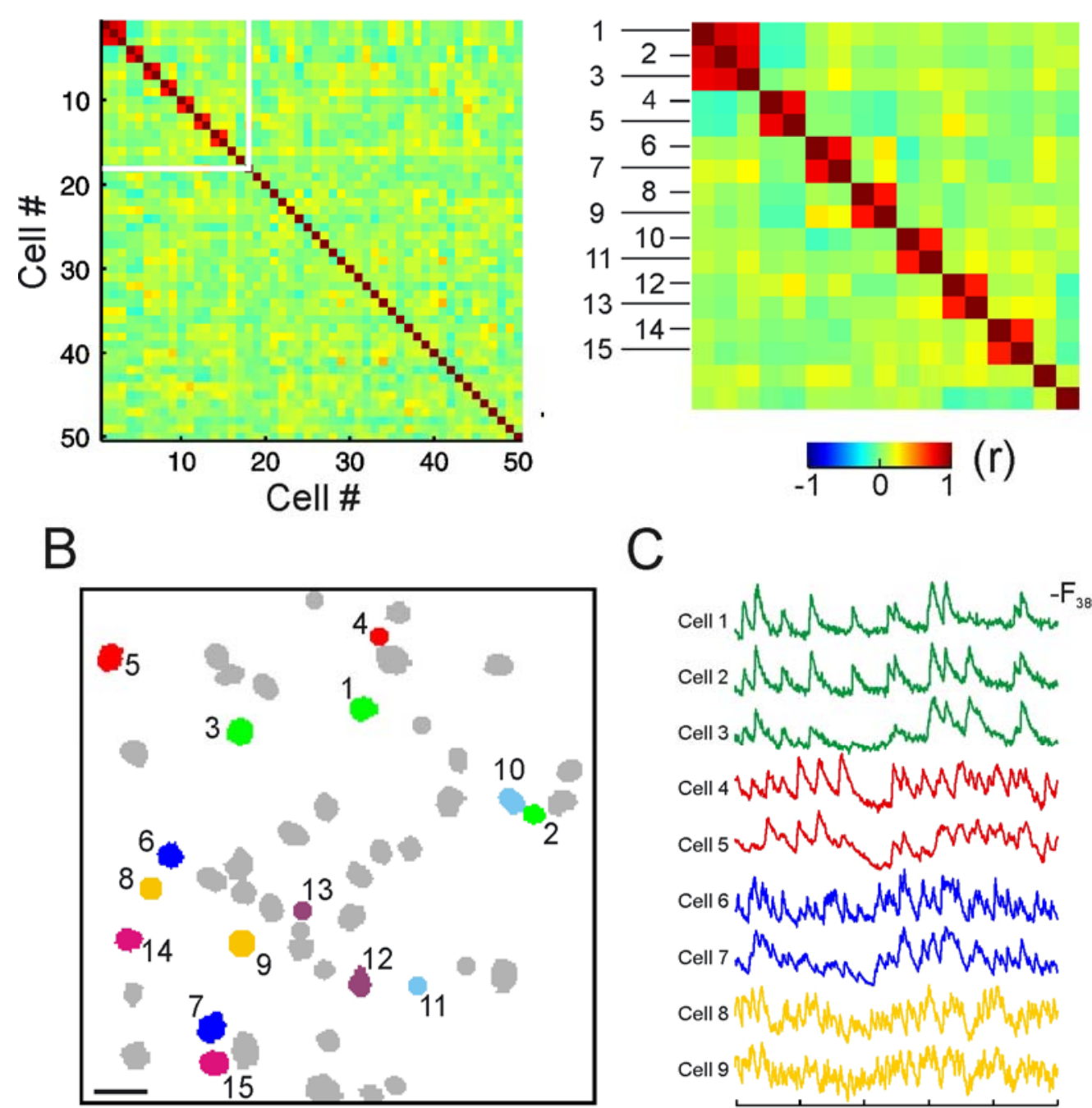

C

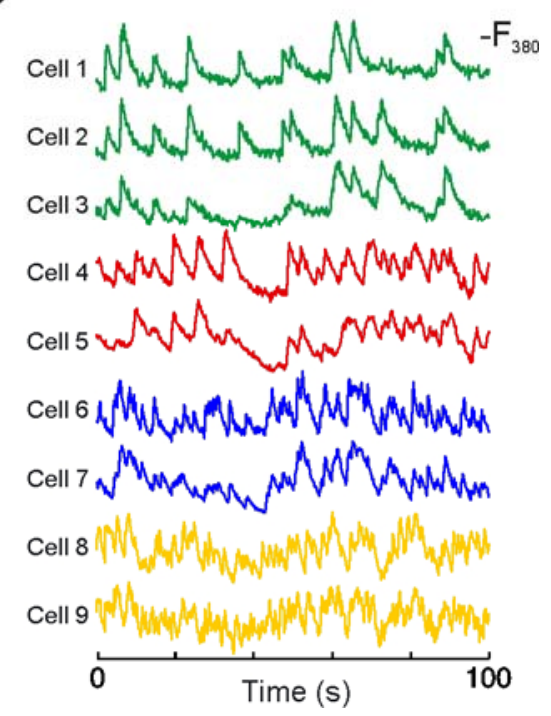

Fig. 3-11 Organization of mitral/tufted cells into multiple "modules" of highly correlated neurons (A) A color coded correlation matrix of the spontaneous $\left[\mathrm{Ca}^{2+}\right]$ activity in 50 simultaneously recorded neurons (same data as in Fig. 3-9). Cells are rearranged according to the correlation values. Distinct regions of high correlation coefficients reveal multiple groups of highly correlated neurons. (B) The spatial locations of the neurons belonging to different modules are shown using different colors. Neurons marked in gray are not correlated to other cells in the same field of view. (C) $\left[\mathrm{Ca}^{2+}\right]$ signals in four representative modules of highly correlated neurons. Scale bar: $20 \mu \mathrm{m}$. 


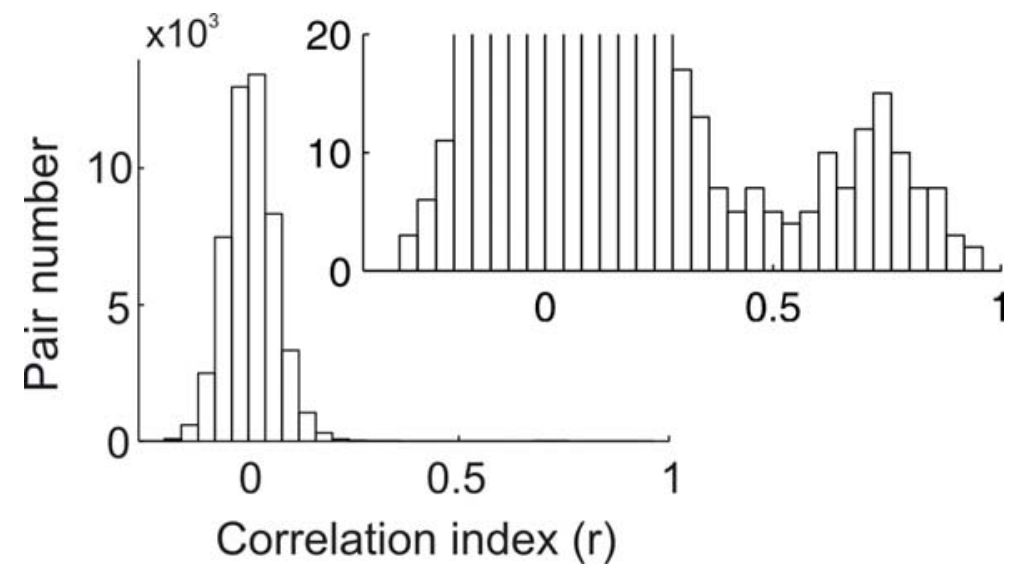

Fig. 3-12 Distribution of the correlation indices (r) of 50325 pairs of OB neurons. Inset: A second peak in the high correlation range indicates the presence of highly correlated cells. A similar peak does not exist in the negative correlation range $(\mathrm{r}<-0.5)$.
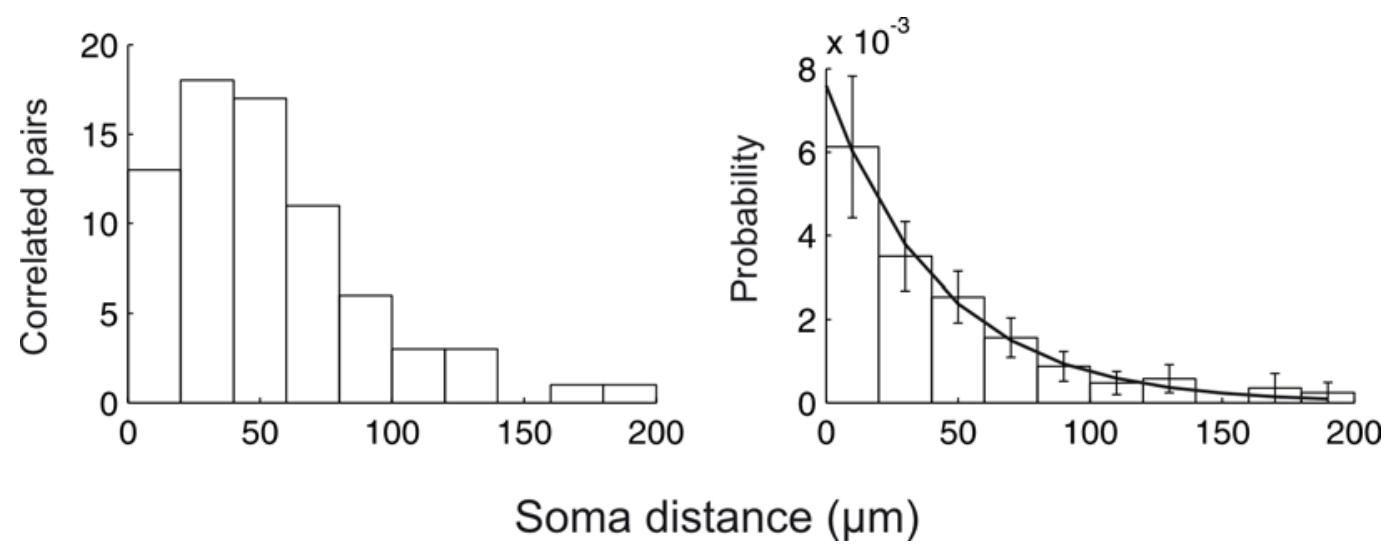

Fig. 3-13 Spatial distribution of highly correlated neurons Left: Histogram of the distances between highly correlated cells ( $r>0.6, n=73$ pairs). Right: The probability of finding correlated neurons as a function of distances. This probability is estimated by normalizing the number of correlated neurons in a given distance bin to the total number of recorded pairs in the same bin. 


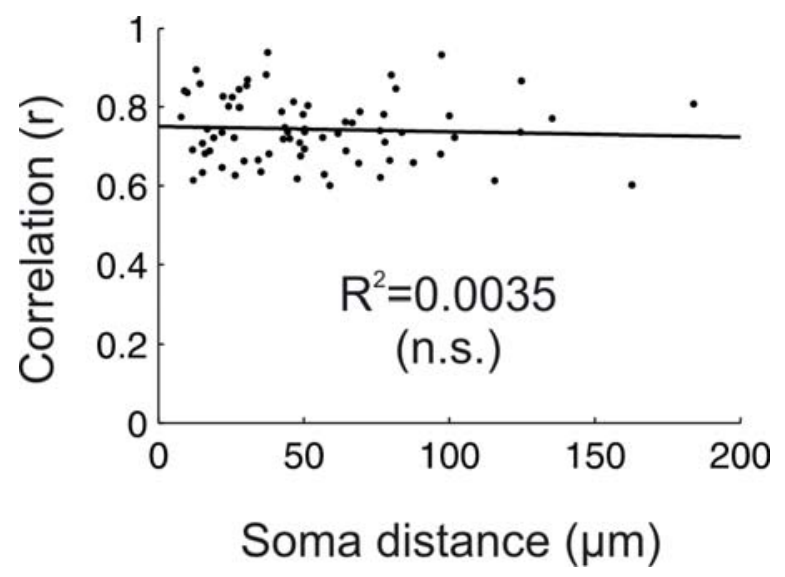

Fig. 3-14 The correlation indices of highly correlated neurons $(r>0.6)$ plotted against their distances. There is no significant dependency between the correlation indices and the distances $\left(\mathrm{R}^{2}=0.0035, \mathrm{P}>0.64\right)$.

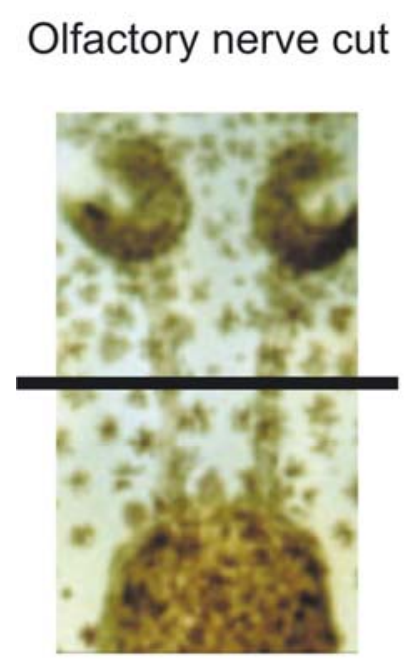

A

B
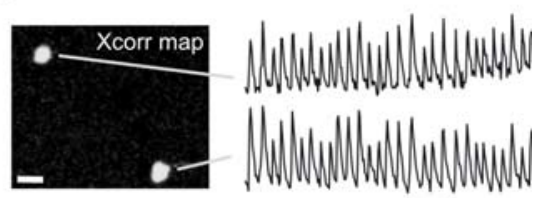
$\mathrm{J}_{10 \mathrm{~s}}^{50 \%}(\Delta \mathrm{F} / \mathrm{F})$

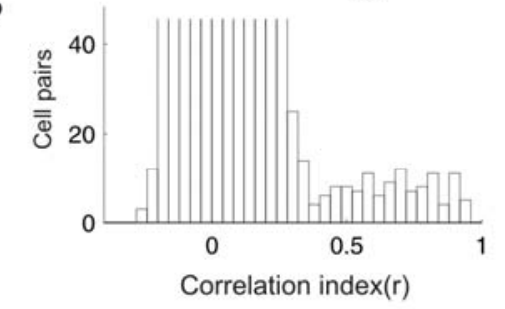

C

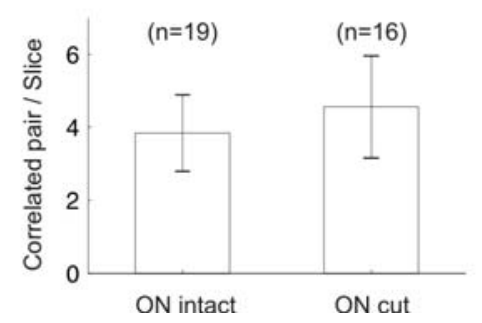

Fig. 3-15 Correlated neurons can be found in preparations with the olfactory nerves (ON) transected. (A) A pair of neurons showing highly correlated activity in the complete absence of receptor inputs. Scale bar, $20 \mu \mathrm{m}$. (B) Distribution of correlation indices in ON-cut preparations (total 31945 cell pairs in 16 optical slices). 74 pairs of neurons exhibit highly correlated activity $(r>0.6)(C)$ The frequency of finding highly correlated pairs in $\mathrm{ON}$-transected preparations did not differ significantly from preparations with intact $\mathrm{ON}(\mathrm{P}>0.66$, t-test). 
To understand whether the correlated activity depend on inputs from olfactory sensory neurons, I performed a set of experiments in preparations with olfactory nerve transected. In these experiments, I carefully cut both the olfactory nerves in the middle (Fig. 3-15, left). To avoid ambiguity, the olfactory epithelia (tissues where the olfactory sensory neurons reside) were completely removed. I then performed $\left[\mathrm{Ca}^{2+}\right]$ imaging in the M/T cell layer of the olfactory bulb. In these experiments, I again detected highly correlated activity in specific pairs of neurons (Fig. 3-15). These cell pairs were found in 14 out of 16 optical slices, with an average of $4.6 \pm 1.4$ pairs/optical slice. The frequency of finding these pairs was not significantly different from the preparations with intact olfactory nerve $(3.8 \pm 1.0$ pairs/optical slice; $n=19$ optical slices; $\mathrm{P}>0.66$ ). These results indicate that the correlated activity is intrinsic to the $\mathrm{OB}$ and does not require inputs from olfactory sensory neurons.

\subsubsection{High temporal resolution analysis of synchronous activity}

I further analyzed the correlated activity of these neurons at a higher temporal resolution using the line-scan mode of the confocal microscope. In these experiments, I first imaged a population of $\mathrm{M} / \mathrm{T}$ neurons at a rate of $4 \mathrm{~Hz}$. I then analyzed these data online and identify neurons showing highly correlated activity. Using this information, I placed a scan line across a specific pair of highly correlated neurons (Fig. 3-16). Scanning the laser focal point repeatedly along this line allowed me to sample the $\left[\mathrm{Ca}^{2+}\right]$ signal of these neurons at a much high temporal resolution. Fig. 3-16 shows an example of such experiments. Individual $\left[\mathrm{Ca}^{2+}\right]$ transients of the two neurons appears to be precisely synchronous to each other, although there are clearly some $\left[\mathrm{Ca}^{2+}\right]$ transients that appear in one cell but are lacking in the other cell. 

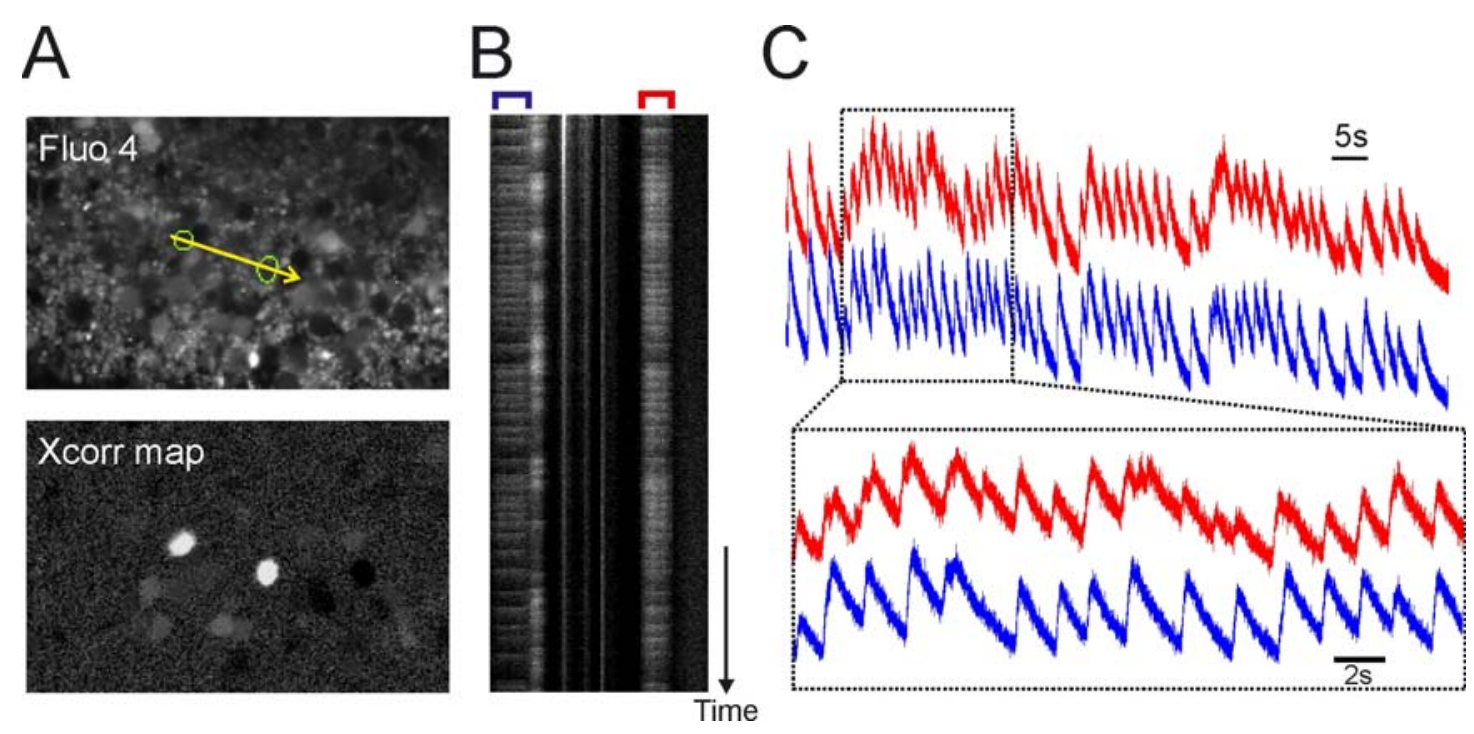

Fig. 3-16 Correlated activity analyzed at a higher temporal resolution (A) A pair of highly correlated neurons identified in the correlation map (below). A scan line placed across the two neurons (yellow line, top). Scanning the laser focal point repeatedly along this line allows recoding the activity of these neurons at a much higher sampling rate. (B) The fluorescence along the scan line plotted over time. The locations of the two neurons marked on the top. (C) High temporal resolution $\left[\mathrm{Ca}^{2+}\right]$ signals of the two cells. Individual $\left[\mathrm{Ca}^{2+}\right]$ transients of the two neurons appear to be precisely synchronous to each other, although there are clearly some $\left[\mathrm{Ca}^{2+}\right]$ transients that appear in one cell but are lacking in the other cell.

To further quantify the degree of spiking synchronization between the correlated neurons. Dr. Bei-Jung Lin performed dual on-cell recordings that specifically targeted cell pairs that showed highly correlated $\left[\mathrm{Ca}^{2+}\right]$ activity. Fig. $3-17$ shows an example of such experiment. Consistent with $\left[\mathrm{Ca}^{2+}\right]$ imaging data, the spike trains of the two neurons were highly correlated. The correlated spikes did not have a fixed delay. The two neurons could either fire nearly simultaneously or with a small positive or negative time lag $(\Delta \mathrm{t})($ Fig. 3-17, B). The distribution of $\Delta \mathrm{t}$ showed a narrow peak near $\Delta t=0($ Fig. 3-17, C), indicating that spikes of one neuron tended to occur within a narrow time window around the spikes of the other neuron and vice versa. A Gaussian fit to the distribution, peaked, on the average, at $3.5 \pm 1.6 \mathrm{~ms}(\mathrm{n}=4)$ 
with a standard deviation of the fitted curves of $13.9 \pm 3.6 \mathrm{~ms}(\mathrm{n}=4)$. These results show that these neurons fire correlated APs at a precision of a few milliseconds.

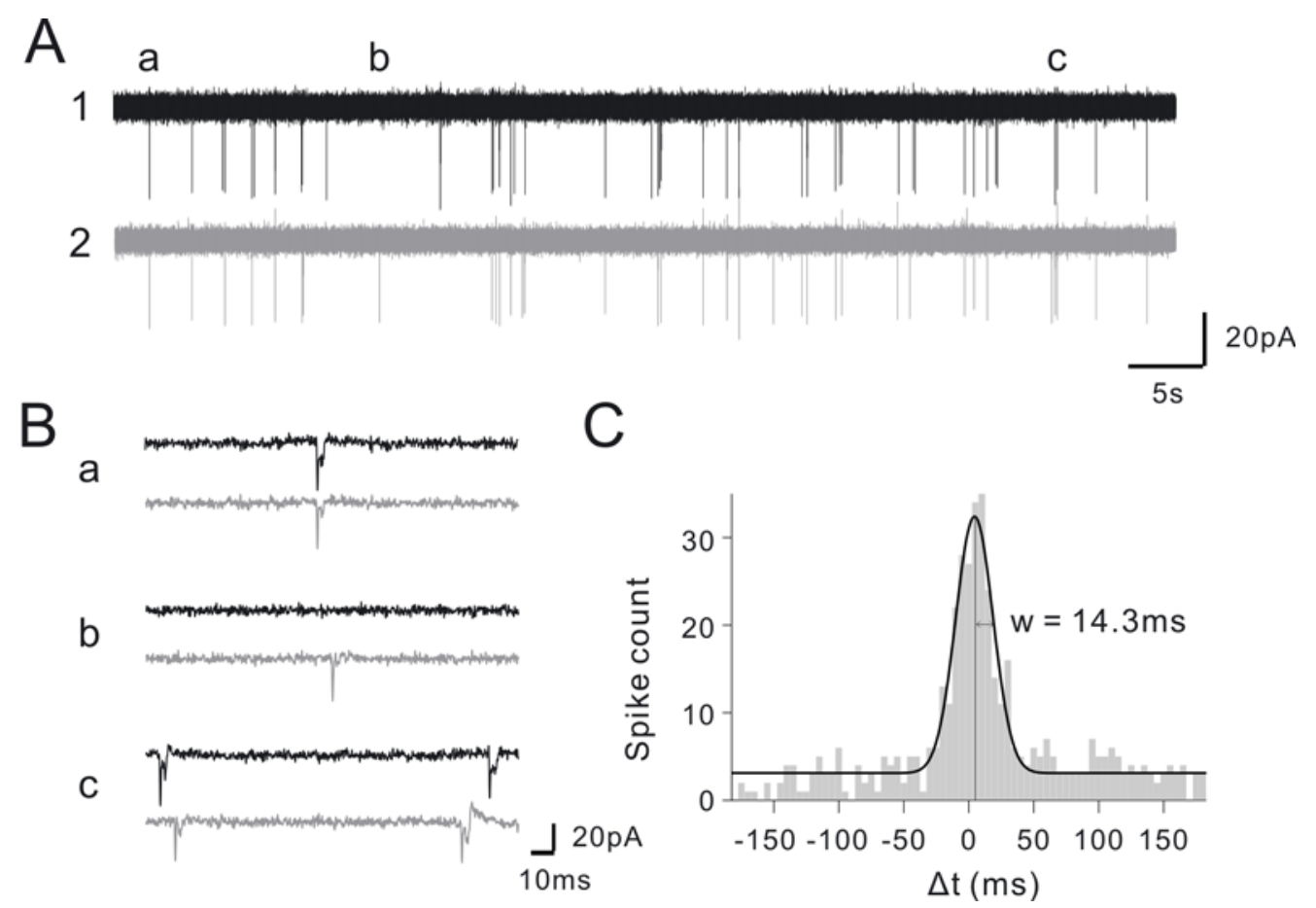

Fig. 3-17 Spiking patterns in neurons showing highly correlated $\left[\mathrm{Ca}^{2+}\right]$ activity

(A) Simultaneous on-cell recording of action potentials in a pair neurons showing highly correlated $\left[\mathrm{Ca}^{2+}\right]$ activity. (B) Expanded traces from regions marked in A. (C) Spike cross-correlogram showing the distribution of the lag $(\Delta t)$ in spike time between the two cells. Experiments performed by Dr. Bei-Jung Lin. 


\subsection{Connections of synchronous neurons into the same glomerulus}

\subsubsection{Evidences from tracer injection}

The data presented so far indicates that the OB contains distinct groups of neurons showing synchronous electrical and $\left[\mathrm{Ca}^{2+}\right]$ activity in the absence of any stimulation. Neurons belonging to a synchronous group are sparsely distributed in the $\mathrm{OB}$ and are intermingled with neurons belonging to other synchronous group. The highly correlated activity suggests that there might be some connections between the synchronous neurons. However, because these neurons can often be located far away from each other, it is difficult to trace their connections in transmission images or in fluorescence images using Fura-2 staining. To understand whether and how these neurons might connect to each other, I performed experiments in which I specifically stained pairs of synchronous neurons with fluorescence dyes using patch pipettes.

In these experiments, I first performed $\left[\mathrm{Ca}^{2+}\right]$ imaging experiments using Fura-2. I then analyzed the data online to identify M/T cell pairs showing synchronous activity. I then selected one specific pair of synchronous M/T cells and re-identified the same neurons under transmission images. Finally, I established whole-cell patch-clamp from each of the synchronous neurons to fill them with fluorescence dyes. To be able to clearly distinguish the processes of the two neurons, I injected the two neurons using fluorescence dyes of different colors (Red: Alexa 555, Green: Alexa 488). Although the emission band of Alexa-488 overlaps that of Fura-2, the initial Fura-2 staining does not interfere with the detection of Alexa 488 because their excitation spectra are quite different (Fig. 3-18; Fura-2 is best excited at 340-380 $\mathrm{nm}$, depending on the $\left[\mathrm{Ca}^{2+}\right]$ level whereas Alexa 488 is best excited at $\left.488 \mathrm{~nm}\right)$. Finally, to avoid ambiguity, only two neurons (one color each) were subjected to staining attempts in each bulb. 


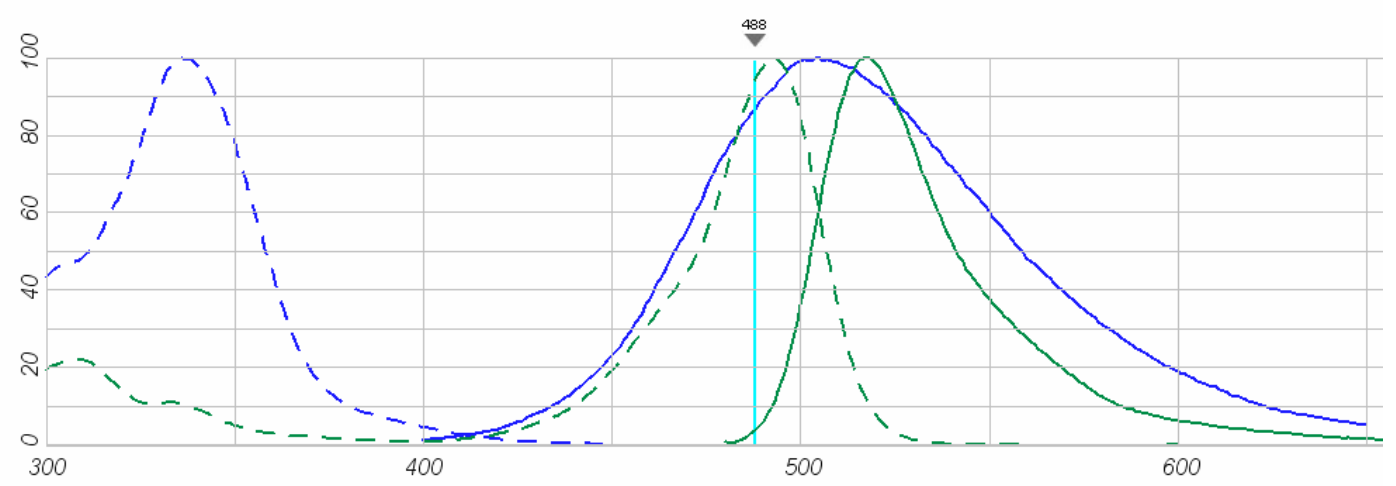

Fig. 3-18 Staining with Fura-2 does not interfere with the detection of Alexa 488 Fluorescence spectra of Fura-2 ([Ca $\left.{ }^{2+}\right]$ bound form, blue curves) and Alexa 488 (Green curve). Dash lines show excitation spectra, solid lines show emission spectra. (Image taken from www.molecularprobes.com)

Fig. 3-19 shows one example (and actually the first successful example) of such experiment. Synchronous M/T cells were identified based on a clear labeling in the correlation map (Fig. 3-19, A) as well as the highly correlated $\left[\mathrm{Ca}^{2+}\right]$ signals of the two neurons (Fig. 3-19, B). The morphological reconstruction of the neurons clearly showed some dendrites connecting the glomerular layer and terminating in tufted-like structures, confirming that these neurons were indeed M/T cells. Strikingly, I observed that the synchronous neurons connected precisely into the same glomerulus. In the z-projection images (Fig. 3-19, C), the dendrites of the two neurons appeared to have some additional contacts than those at their overlapping dendritic tufts. However, a careful examination of the neurons' dendritic branching pattern in 3D showed that, in most cases, the non-tuft dendrites of the two cells were not actually touching each other. There were only two locations in this case where the separation of the neurons' processes cannot be determined unambiguously (arrows in Fig. Fig. 3-19, D). However, in a different example, I found that the processes of the two correlated neurons clearly separated from each other with the only putative contacts being located in the shared glomerulus (Fig. 3-20). This suggests that the intra-glomerular 
contacts of the two neurons play an important role in their correlated activity. There were some cases where the synchronous neurons' primary dendrites terminated in more than one dendritic tuft (Fig. 3-21). In these cases, the neurons' dendritic tufts can overlap at precisely the same two locations (Fig. 3-21).

I performed such morphological reconstruction experiments in a total of 9 pairs of synchronous neurons $(r>0.6)$. The distances between these neurons ranged from $20-109 \mu \mathrm{m}$ (average: $57.3 \mu \mathrm{m}$ ). In all cases, the two synchronous neurons invariably had dendrites that connect to the same glomerulus with at least one overlapping dendritic tuft (Table 3-1). In contrast, neurons showing low correlation $(r<0.4)$ connect to different glomeruli $(n=3)$. This evidence, together with the fact that the overlapping glomerular tufts are sometimes the only place where the two neurons come within contact (e.g. Fig. 3-20 A), suggests that the connections of the neurons to the same glomerulus plays an important role in their correlated activity. 
A
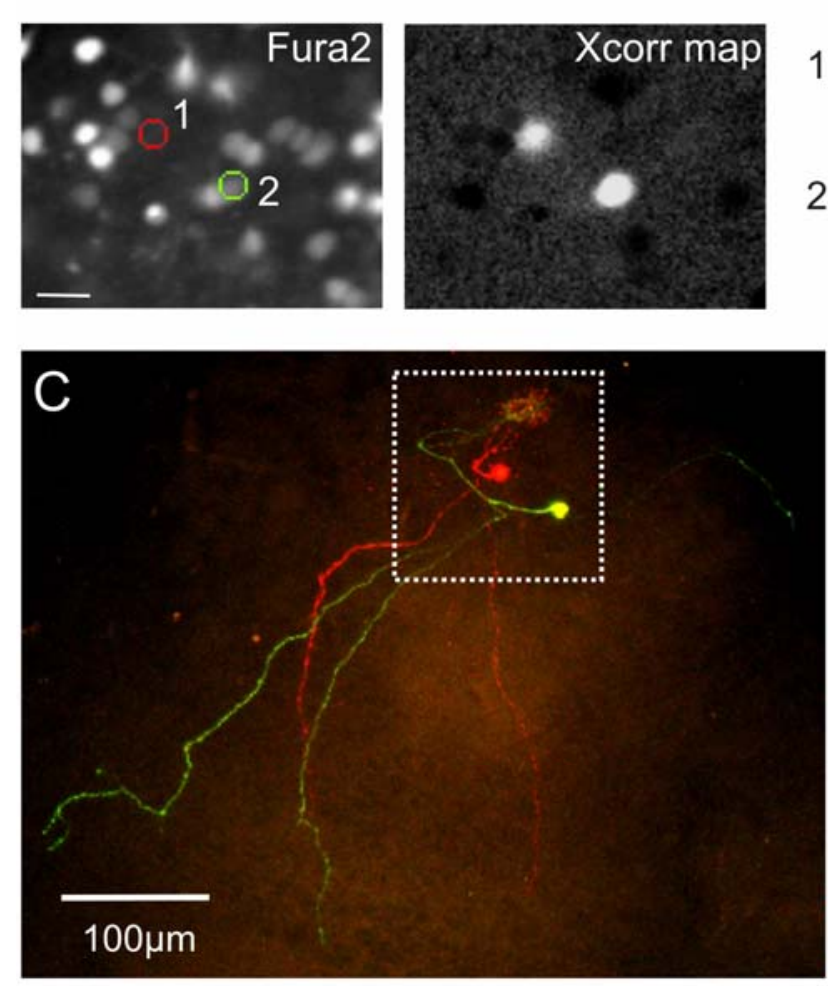

B
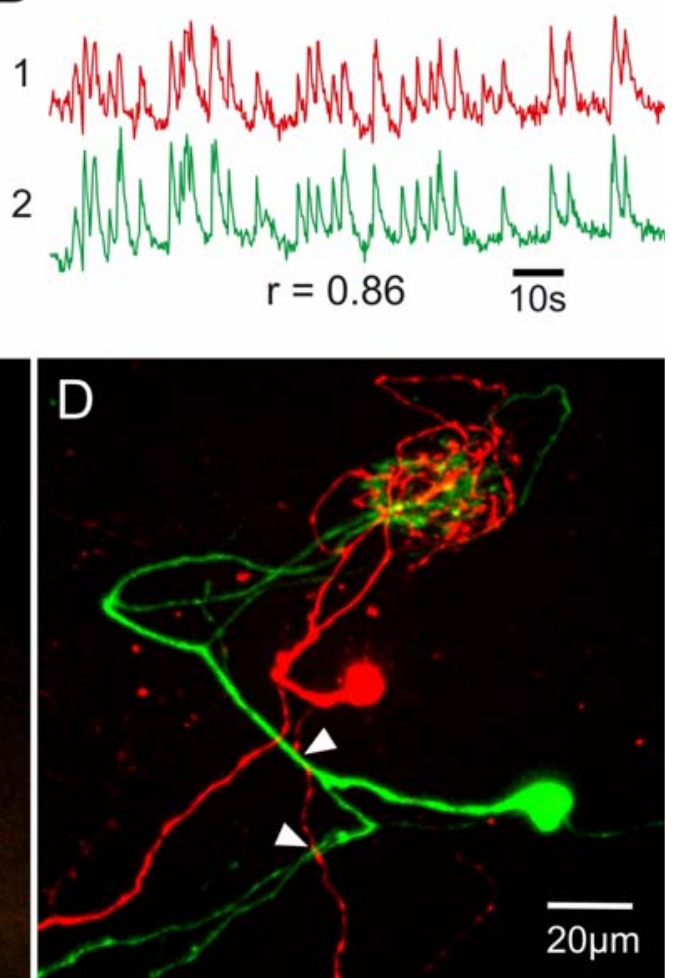

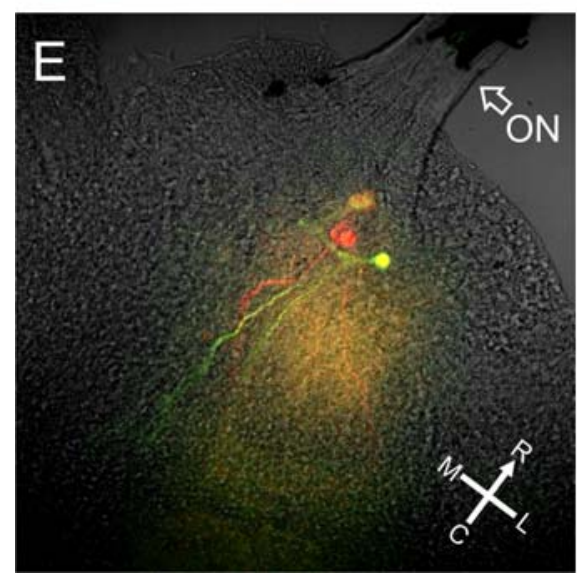

Fig. 3-19 Morphological reconstruction of synchronous neurons (A) A pair of synchronous neurons identify in the cross-correlation map. Scale bar, $20 \mu \mathrm{m}$ (B) The $\left[\mathrm{Ca}^{2+}\right]$ activity of these two neurons. (C) Morphology of the neurons reveals by dye injection. These neurons issue several dendrites. Some of these dendrites connect to the glomerulus while others extend several hundred micrometers caudally into the granule cell layer. (D) The dendritic tufts of the two neurons branch precisely within the same glomerulus. (E) Overlay of the fluorescence image and the transmission image showing the orientation of the slice. ON: olfactory nerve. 

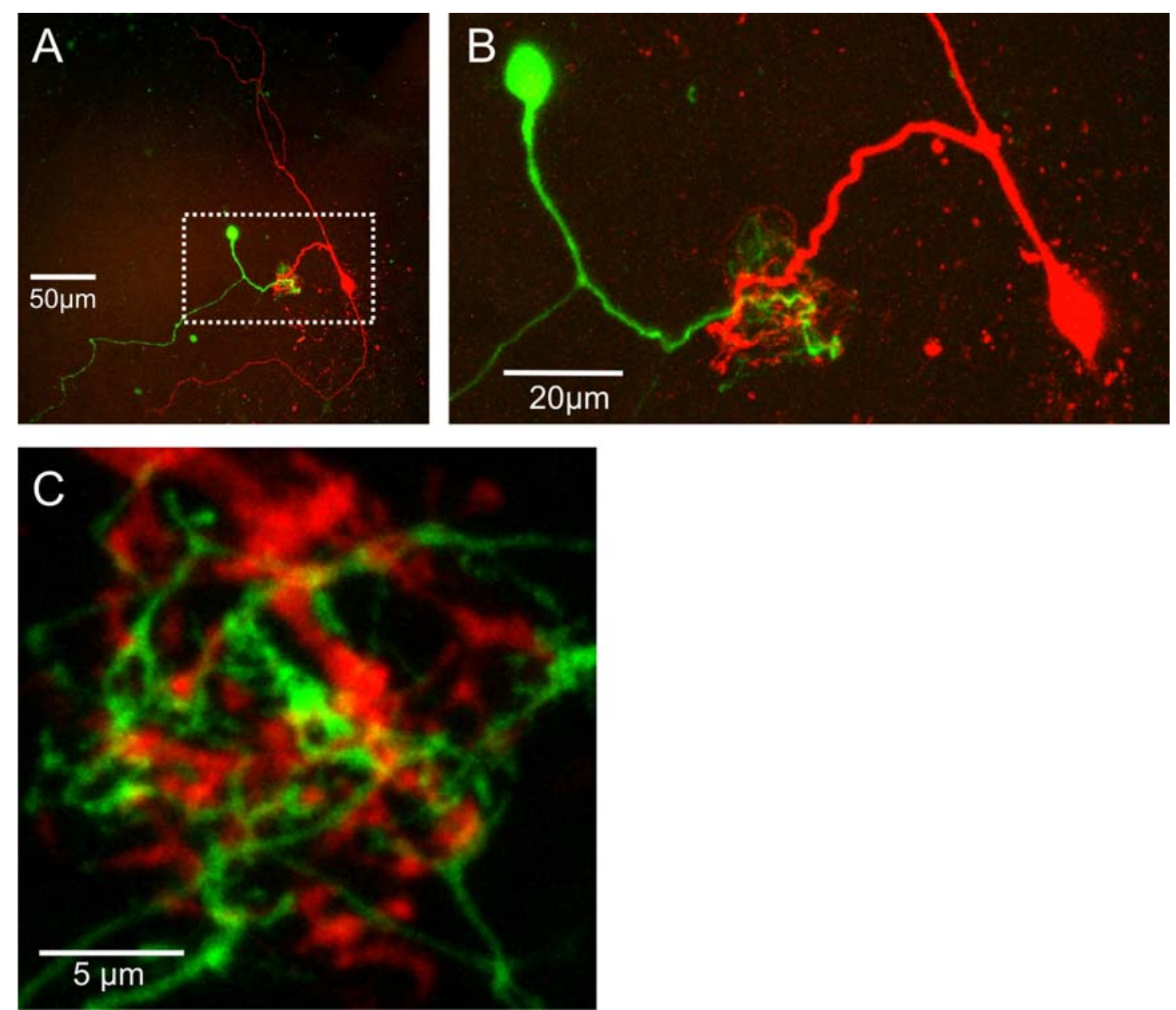

Fig. 3-20 Morphological reconstruction of synchronous neurons (More examples) (A) Overview of the dendritic morphology of the two cells at a lower magnification. The processes of these two neurons do not touch each other except within the shared glomerulus. (B) A magnified view of the glomerular connection of the two neurons. (C) The branching pattern of two synchronous neurons within the shared glomerulus. This image is taken from yet another pair of synchronous neurons. 


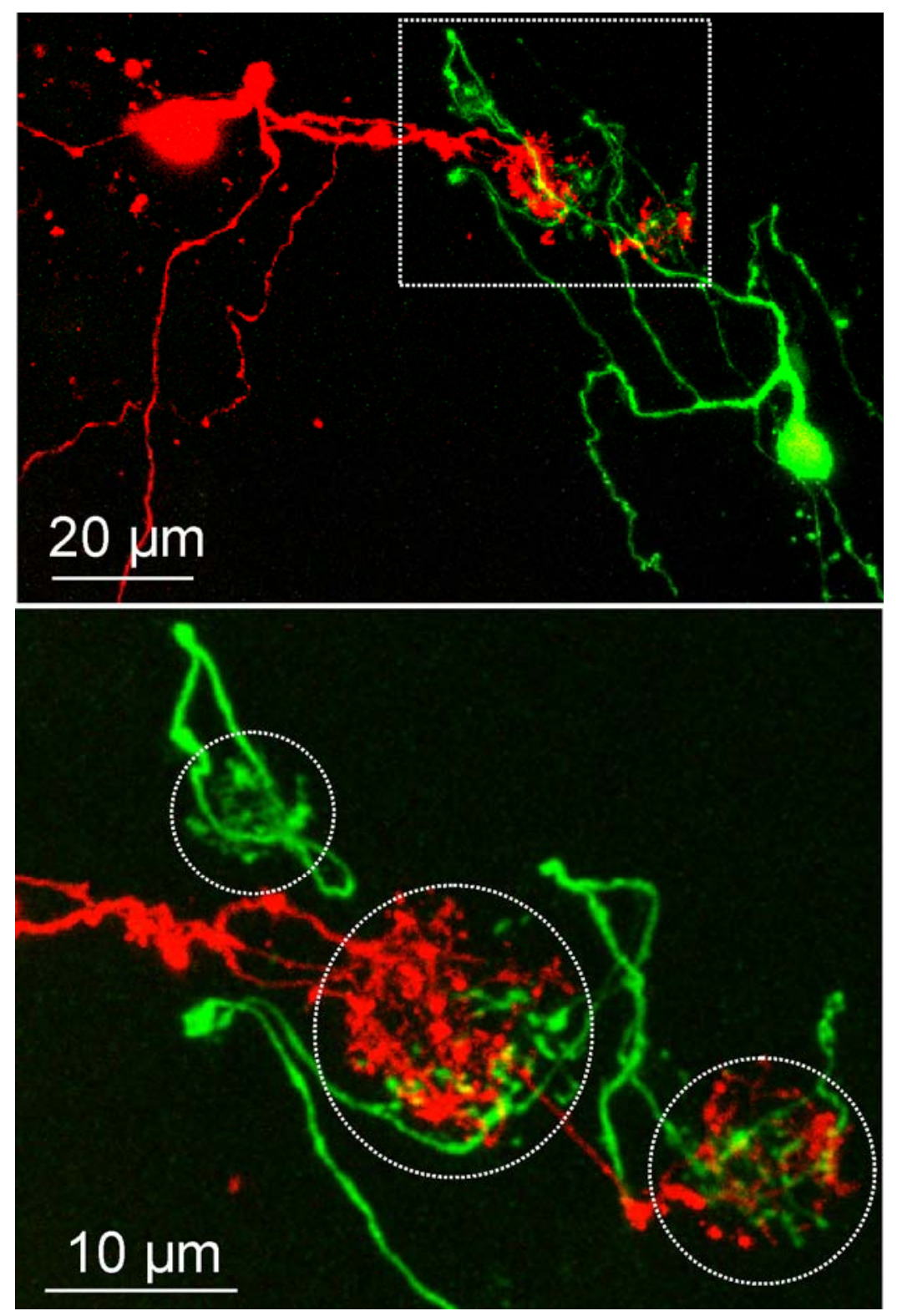

Fig. 3-21 A pair of synchronous neurons whose dendrites contact in the glomerular layer at precisely the same two locations. The green neuron shows an additional dendritic tuft not shared by the red neuron. 


\begin{tabular}{cccccl}
\hline \hline Pair ID & $\begin{array}{c}\text { \# tufts } \\
\text { (cell1) }\end{array}$ & $\begin{array}{c}\text { \# tufts } \\
(\text { cell2) }\end{array}$ & $\begin{array}{c}\text { Overlap } \\
\text { tufts }\end{array}$ & $\begin{array}{c}\text { Distance } \\
(\mu \mathrm{m})\end{array}$ & \\
\hline 1 & 2 & 1 & 1 & 20.1 & \\
2 & 1 & 1 & 1 & 25 & \\
3 & 1 & 1 & 1 & 30 & Fig. 3-9 \\
4 & 2 & 2 & 2 & 44.6 & \\
5 & 1 & 1 & 1 & 50 & \\
6 & 1 & 1 & 1 & 70 & \\
7 & 1 & 1 & 1 & 73 & Fig. 3-20 (C) \\
8 & 1 & 1 & 1 & 94.2 & Fig. 3-20 (A,B) \\
9 & 3 & 2 & 2 & 109.2 & Fig. 3-21 \\
\hline \hline
\end{tabular}

Table 3-1 Summary of the morphological reconstruction experiments of 9 pairs of synchronous neurons (r>0.6). In all cases, the two neurons invariably show at least one overlapping dendritic tufts within the glomerulus. 


\subsubsection{Evidence from cross-correlation maps}

The dye injection experiments presented in the previous section allow visualizing the dendritic morphology of two neurons of a synchronous module. To visualize a complete module, one would need to identify more synchronous neurons and fill each of them with fluorescence dye. With the number of neurons, this experiment would become increasingly difficult to carry out. In Sec 3.1, I have shown that the activity cross-correlation map (CCM) allows a high contrast "virtual labeling" of OB neurons. Moreover, it is possible to trace the dendritic connections of M/T cells to the glomerulus in the CCM (Fig. 3-7). In this section, I present experiments in which I attempt to reconstruct the dendritic connection of a substantial part of a synchronous module by reconstructing CCMs of the same module at different z-positions.

In these experiments, I imaged the spontaneous activity of OB neurons at different z-positions using a high resolution objective (40X/1.3). The gaps between adjacent z-planes were carefully chosen to be slightly smaller than the thickness of the optical slice. I reconstructed CCMs using the $\left[\mathrm{Ca}^{2+}\right]$ signals of neurons as reference waveforms. As before, individual CCMs often labeled other neurons and processes that were synchronous to the reference neuron (Fig. 3-22 A, left). Due to the overlap between optical slices, the same cells or the same dendritic compartments could be detected in adjacent z-planes, so that I can reliably select regions of interest belonging to the same cell in the next z-plane (Fig. 3-22 A). This allowed me to measure a new reference trace and construct CCM of the same module at this z-position. Carrying out the same procedure for all subsequent planes led to a labelling of all neurons and processes in $3 \mathrm{D}$ that exhibit synchronous activity to the reference cell. 
Fig. 3-22 B shows a maximal z-projection of all obtained CCM of the same module. This map labelled a total of 4 neurons. Except for one neuron whose dendrites went out of the observation volume, all other cells had dendrites that connected to the same glomerulus. This data is consistent with the dye injection experiments presented in the previous section, suggesting that all neurons of a synchronous module share the same glomerular connection.

I reconstructed the 3D CCM of a number of other neuronal modules within the same observation volume. These maps were coded using different colors and overlaid on the same image (Fig. 3-22, C). Notably, neurons coded using different colors (i.e. differently correlated neurons) invariably connected to different glomeruli. This further suggests that M/T neurons exhibit synchronous activity if and only if they share the same glomerular connections. 


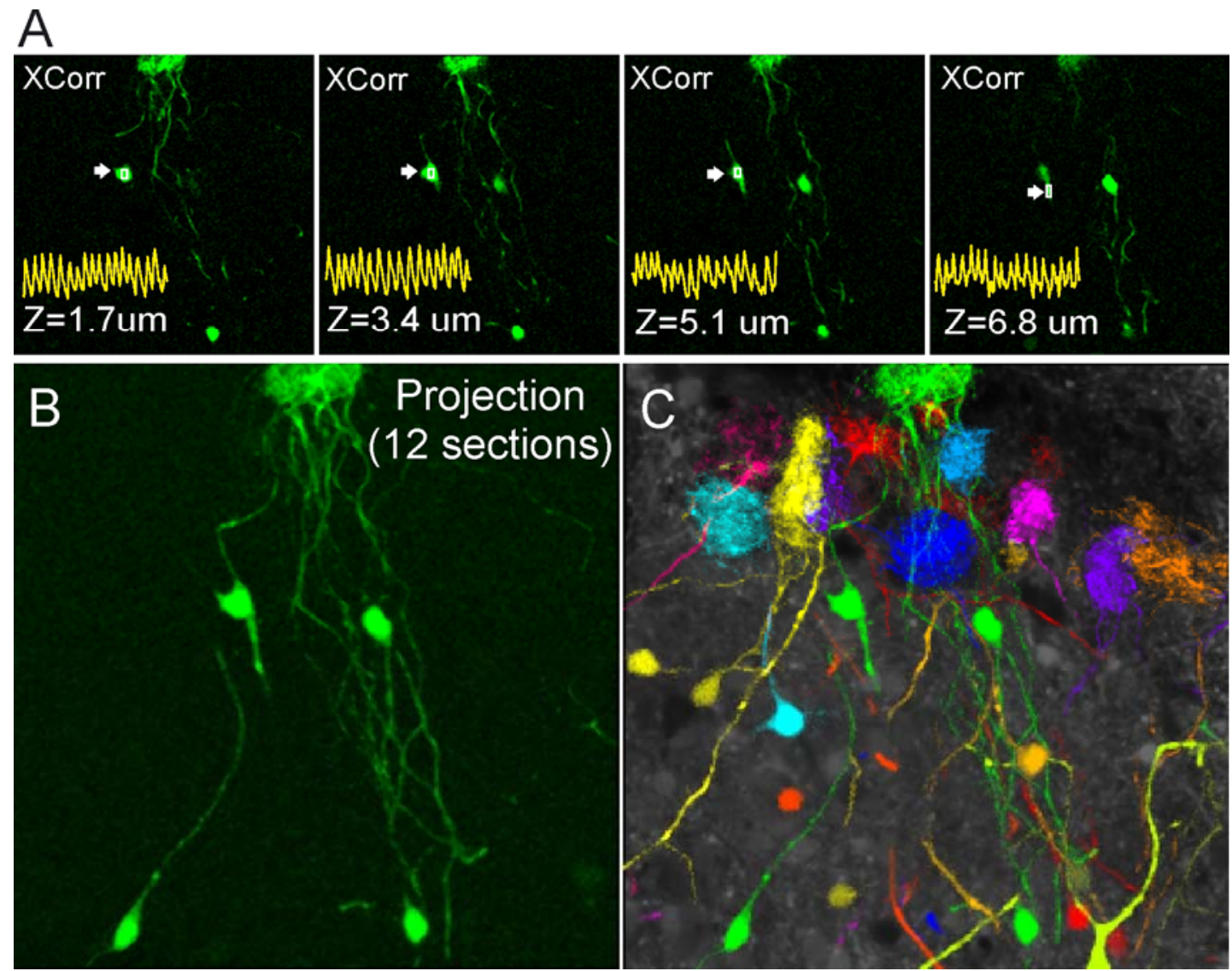

Fig. 3-22 Visualizing the dendritic connections of synchronous modules (A)

Correlation maps of one neuron at different z-positions reconstructed by sequentially acquiring time-series of confocal images at different z-planes. An overlap between optical slices allows the selection of ROIs (arrows) belonging to the same cell at different z-planes. The reference signals used to generate the corresponding correlation maps were superimposed. (B) Maximum z-projection of the correlation maps of this cell reveals the dendritic connections of synchronous neurons into the same glomerulus (C) Superposition of different correlation maps using different colors. Neurons coded with different colors (i.e. differently correlated neurons) invariably connect to different glomeruli. 


\subsection{Characterization of sensory responses in synchronous neurons}

The reliable relationship between the synchronization of spontaneous activity and common glomerular connection offers a highly specific criterion for identifying neurons that connect to the same glomerulus. This means that, by analyzing the correlated spontaneous activity of $\mathrm{M} / \mathrm{T}$ cells, one can reliably predict whether a pair of $\mathrm{M} / \mathrm{T}$ cells connects to the same glomerulus. In this part of the thesis, I specifically used this relationship as a tool to investigate a long standing question in olfaction, how the odor representation of M/T cells can be related to the neurons' glomerular connectivity.

\subsubsection{General properties of odor responses in the OB}

The odor representation of $\mathrm{M} / \mathrm{T}$ cell population has been extensively analyzed in different species using a variety of methods ranging from intra-cellular recordings (Hamilton and Kauer, 1989;Luo and Katz, 2001;Cang and Isaacson, 2003), extra-cellular recordings (Buonviso and Chaput, 1990;Kashiwadani et al., 1999;Friedrich and Laurent, 2001;Friedrich et al., 2004), and optical imaging (Cinelli et al., 1995; Yaksi et al., 2007). Although none of these studies have related the recorded responses to the neurons' glomerular connectivity, these studies yielded a common picture of $\mathrm{M} / \mathrm{T}$ cells' odor coding as an active process with individual neurons showing complex and dynamic spatio-temporal responses. The odor representations of Xenopus M/T cells have recently been analyzed by Dr. Bei-Jung Lin in our lab using a combination of electrophysiology and $\left[\mathrm{Ca}^{2+}\right]$ imaging approaches. In her study, she shows that a single odor can elicit different $\left[\mathrm{Ca}^{2+}\right]$ response patterns in different $\mathrm{M} / \mathrm{T}$ cells, and these $\left[\mathrm{Ca}^{2+}\right]$ responses can be generally categorized as excitatory or inhibitory responses (Lin et al., 2007). Moreover, the excitatory or inhibitory $\left[\mathrm{Ca}^{2+}\right]$ responses correspond to an increase or a suppression of 
firing rates of these neurons (Lin et al., 2007).

I first repeated the $\left[\mathrm{Ca}^{2+}\right]$ imaging experiments of odor responses as described in (Lin et al., 2007). Fig. 3-23 shows an example in which I recorded the $\left[\mathrm{Ca}^{2+}\right]$ activity of $\mathrm{M} / \mathrm{T}$ cells in response to odor stimulations applied to the ipsilateral olfactory epithelium. In line with the previous observation, the $\left[\mathrm{Ca}^{2+}\right]$ activity of these neurons were clearly modulated in at least two different ways. In some cells (e.g. cell $1,2)$, odor induced reliable $\left[\mathrm{Ca}^{2+}\right]$ increases that could be clearly distinguished from their spontaneous activity. In other cells (e.g. cell 3, 4), odor suppressed spontaneous $\left[\mathrm{Ca}^{2+}\right]$ transients, leading to an overall $\left[\mathrm{Ca}^{2+}\right]$ decrease with respect to the pre-stimulus level. I quantified the responses of 448 cells ( 9 bulbs) to an odorant stimulus (mixture of 14 amino acids, see Materials and Methods). An excitatory or inhibitory response was assumed when the fluorescence change within a 7 s post-stimulus window exceeded $\pm 2.5 \mathrm{SD}$ of the spontaneous fluctuations. Using this criterion, $29.5 \%$ of the examined neurons showed excitatory responses and $14.7 \%$ showed inhibitory responses.

The spatial distribution of odor-responsive M/T cells was highly heterogeneous (Fig. 3-23 A). There was a substantial intermix of neurons that showed excitatory responses with others showing inhibitory responses. In 177 randomly chosen pairs of neighboring and odor-responsive neurons, 57 pairs responded in an opposite way (i.e., excitation in one cell and inhibition in the other one). In cases where both responses were either excitatory or inhibitory, the durations or response waveforms would differ (Fig. 3-23 B). 
A

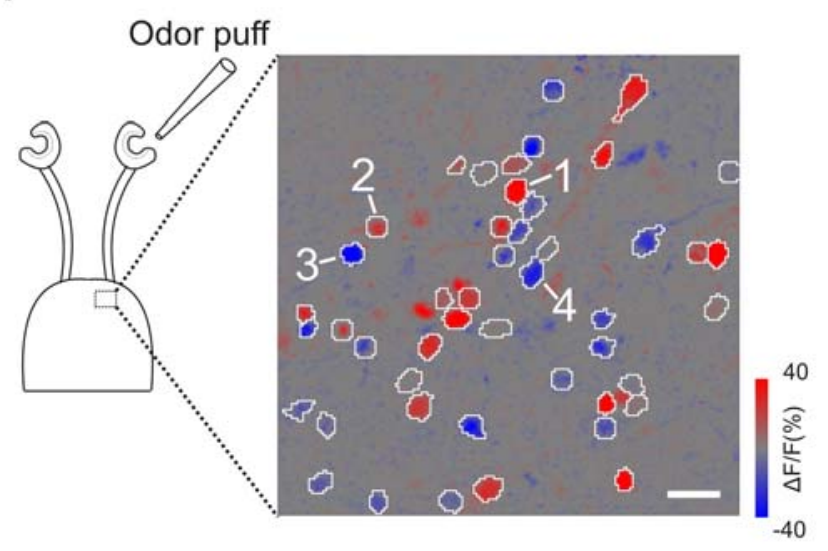

B

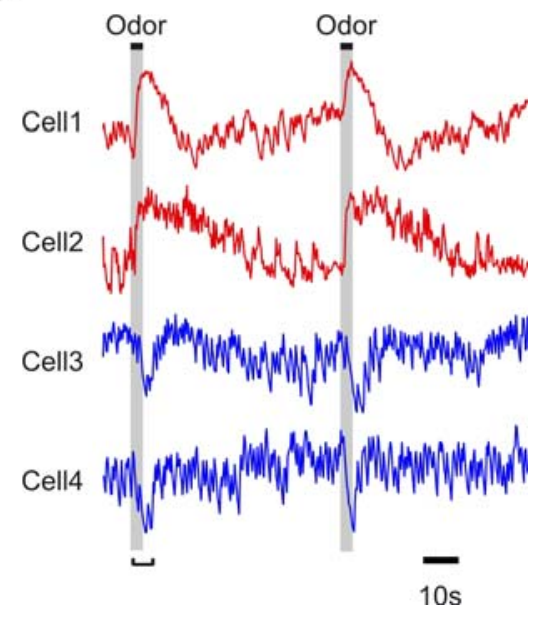

Fig. 3-23 Odor-induced patterns of excitatory or inhibitory responses in M/T cells (A) Schematic of a nose-brain-preparation and odor-induced fluorescence changes in the zoomed field of view indicated. The odor responses in a post-stimulus time window (marked in B) color coded and displayed as a spatial map. Cells that are excited (red) or inhibited (blue) are intermingled. Scale bar, $20 \mu \mathrm{m}$. (B) Time courses of $\left[\mathrm{Ca}^{2+}\right]$ signals in four cells before, during and after odor applications. The timing of the odor stimulations are indicated by black bars (above the traces) and extended downward (gray).

\subsubsection{Odor responses in synchronous and non-synchronous neurons.}

As the next step, I analyzed M/T cells' odor responses with respect to their glomerular connectivity as inferred from the correlation between the neurons' spontaneous $\left[\mathrm{Ca}^{2+}\right]$ activity. In these experiments, I first recorded the spontaneous $\left[\mathrm{Ca}^{2+}\right]$ activity in a population of $\mathrm{M} / \mathrm{T}$ cells and subsequently recorded the odor-induced responses of the same neurons. Cross-correlation analysis of the spontaneous data allowed me to identify groups of synchronous neurons as those cells that connect to the same glomerulus. I then specifically analyzed whether there are some differences of the odor response properties in synchronous neurons or non-synchronous neurons. 
Fig. 3-24 shows two examples of such experiments. Neurons with synchronous spontaneous activity were labeled in the correlation maps and their responses to odors were shown as the traces below. In both cases, I observed a strikingly matched odor-induced response in neurons showing synchronous spontaneous activity. In the first example, odor stimulation induced excitatory $\left[\mathrm{Ca}^{2+}\right]$ responses in both cells (Fig. 3-24, left). This is consistent with these neurons sharing common excitatory OSN inputs within the same glomerulus. To my surprise, I found that when odors elicit an inhibitory response, it occurs simultaneously in all neurons of a synchronous module (Fig. 3-24, right). The strong similarity of the odor-induced responses was highly restricted to neurons showing synchronous spontaneous activity. In both examples, when I measured the odor responses from nearby but non-synchronous neurons, these neurons show very different or even opposite responses (Fig. 3-24).

I continued with these experiments and measured the odor-induced responses in a total of 18 modules of synchronous $\mathrm{M} / \mathrm{T}$ cells. In all cases, odor stimulation elicits precisely matched responses in all neurons of a synchronous module. The responses of these neurons were similar not just in direction but also in amplitude, duration and temporal patterns (Fig. 3-25, A). I quantified the response amplitudes and the response waveforms in 18 pairs of synchronous neurons and in 18 non-synchronous neurons located next to one of the synchronous neurons. Although the synchronous neurons were separated by larger distances in all cases (Fig. 3-25, B), their responses, in particular the response directions, amplitudes (Fig. 3-25, C) and the temporal waveforms (Fig. 3-25, D) were more similar than in the nearby, non-synchronous pairs. 

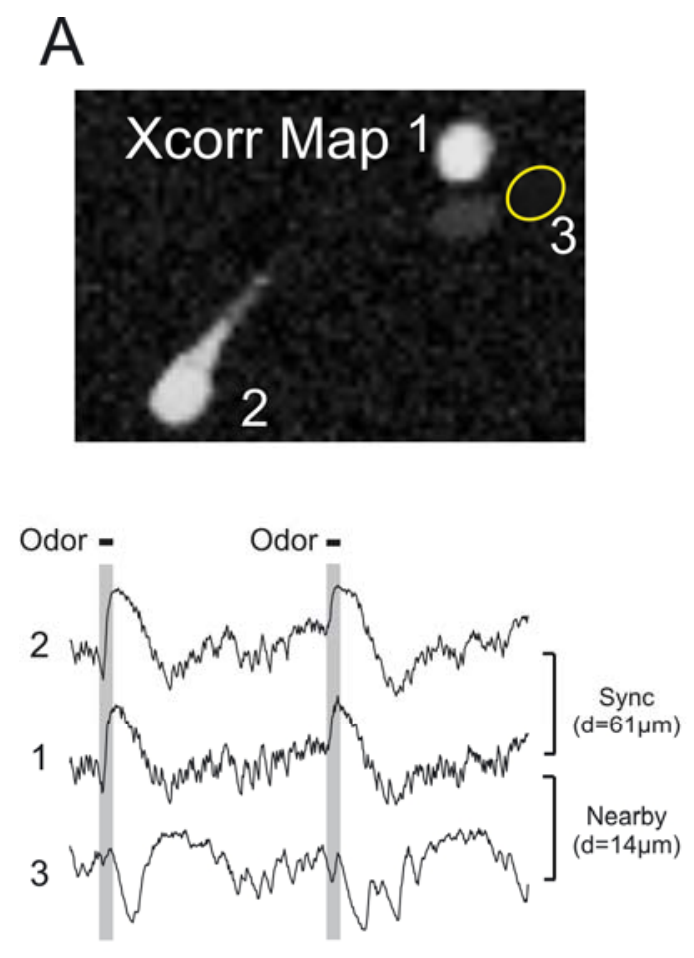

\section{B}
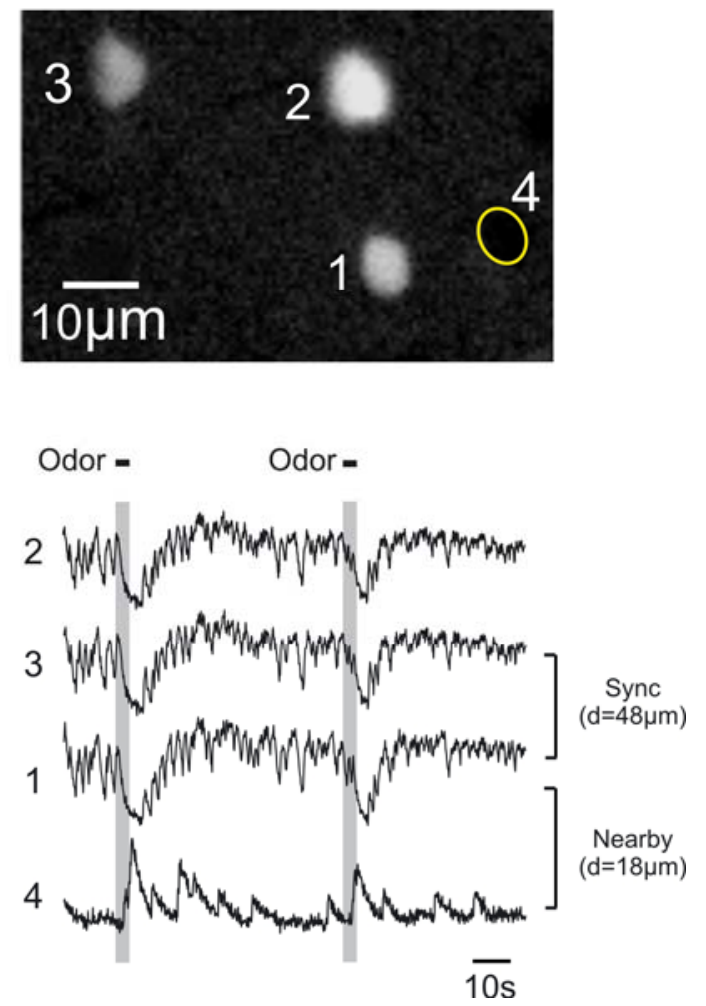

Fig. 3-24 Synchronous neurons show precisely matched excitatory/inhibitory odor responses. (A) A pair of synchronous neurons identified in the correlation map. This pair consistently responds to odor stimulation (mix AA) with an increase in $\left[\mathrm{Ca}^{2+}\right]$. An uncorrelated neuron located next to cell 1 respond in an opposite direction. (B) Another module of three synchronous neurons responded to odor with $\left[\mathrm{Ca}^{2+}\right]$ decrease. An uncorrelated neuron (cell 4) shows an opposite response. 
A

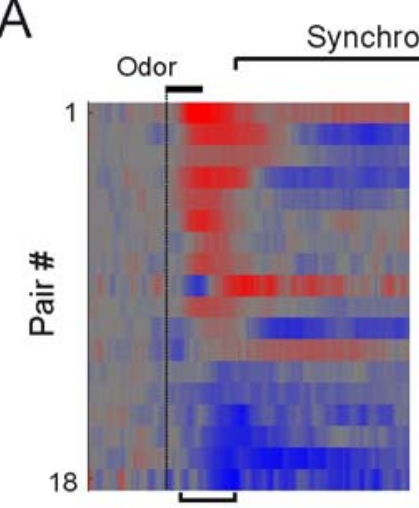

C

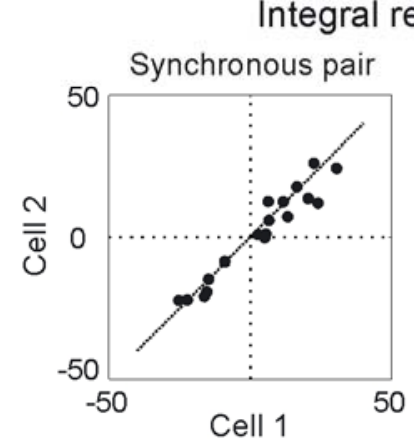

Nearby

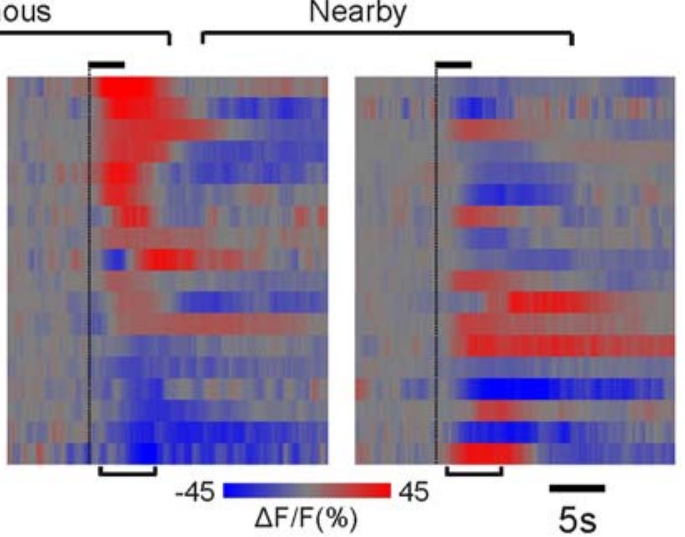

$\mathrm{B}$ Distance $(\mu \mathrm{m})$

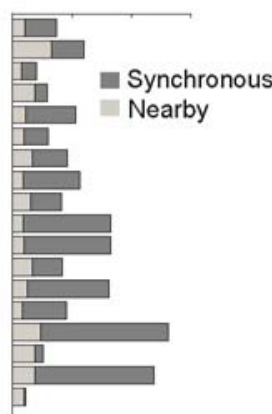

Fig. 3-25 Synchronous M/T cells show precisely matched excitatory/inhibitory odor responses (A) Odor-induced calcium responses color coded and shown for 18 pairs of synchronous $\mathrm{M} / \mathrm{T}$ cells (left and middle panel). The responses of non-synchronous neurons located next to one of the synchronous cells as a control (right panel). (B) The soma distances between the synchronous pairs and the nearby pairs in (A). (C) Integral responses in a post-stimulus time window (marked under the panels in B) plotted for the synchronous cell pairs (left) and the nearby, non-synchronous pairs (right). (D) The correlation coefficient of the response waveforms in synchronous pairs and nearby pairs $\left(\mathrm{n}=18 ;{ }^{*} \mathrm{p}<10^{-4}\right)$. 
The above results suggest that the apparently complex patterns of $\mathrm{M} / \mathrm{T}$ cell responses actually follow a strikingly precise rule. $\mathrm{M} / \mathrm{T}$ cells that connect to different glomeruli (i.e. uncorrelated neurons) can show very different responses even when they are located next to each other, whereas $\mathrm{M} / \mathrm{T}$ cells of the same glomerulus (i.e. synchronous neurons) show precisely matched responses even when they are located far away. This precision is surprising given a complex synaptic interaction within the OB (Chen et al., 2000;Friedrich and Laurent, 2001;Friedrich et al., 2004). I therefore tested whether the similarity of responses of the synchronous neurons holds for other odorants.

In these experiments, synchronous neurons were first identified by analyzing the spontaneous activity as previously described. We then applied a panel of different odorants to the olfactory epithelium and measured the odor responses of M/T cells. To avoid ambiguity, whenever there was a need to apply the same odorant twice, it was always interleaved with the application of different odorants. Further, consecutive odor stimulations were always separated by at least 50 s to avoid possible effects of adaptation.

Fig. 3-26 shows an example of such experiment. For a given neuron, applying the same odors leads to similar responses whereas applying different odors leads to different response patterns (Fig. 3-26, B). This indicates that the responses of individual M/T cells carry information about odor identity. Furthermore, the same neuron can be excited by some odors while being inhibited by other odors, indicating different odors elicit different but odor-specific patterns of excitatory/inhibitory inputs into a particular $\mathrm{M} / \mathrm{T}$ cell.

We next analyzed the response profile of $\mathrm{M} / \mathrm{T}$ neurons with respect to the 
correlation of their spontaneous activity. In nearby but non-synchronous neurons (e.g. cell 2 and cell 3 in Fig. 3-26, A and B), the responses can be similar for some odorants (e.g., the amino acid mixture inhibited both cell 2 and 3) but different for others (e.g., lysine excited cell 2 but inhibited cell 3). However, in neurons belonging to the same synchronous module (cell 1, 2 or cell 3,4), different odorants led to virtually identical patterns of excitatory/inhibitory responses (Fig. 3-26, B and C).

A

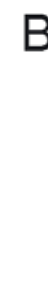

Spontaneous

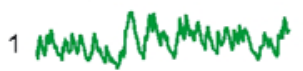

2 Midw NWWWWH

3 MWNMWMN

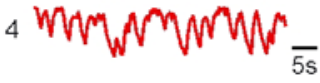
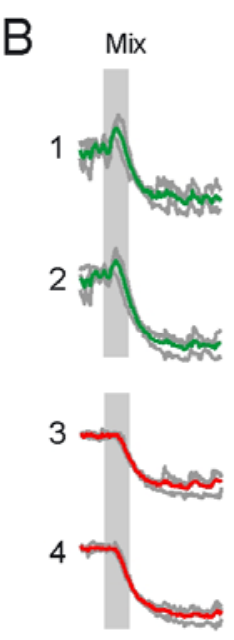
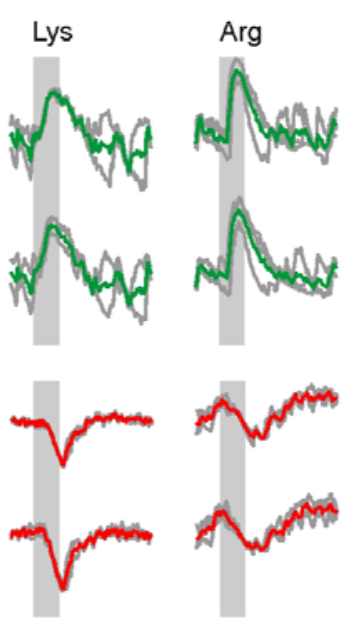

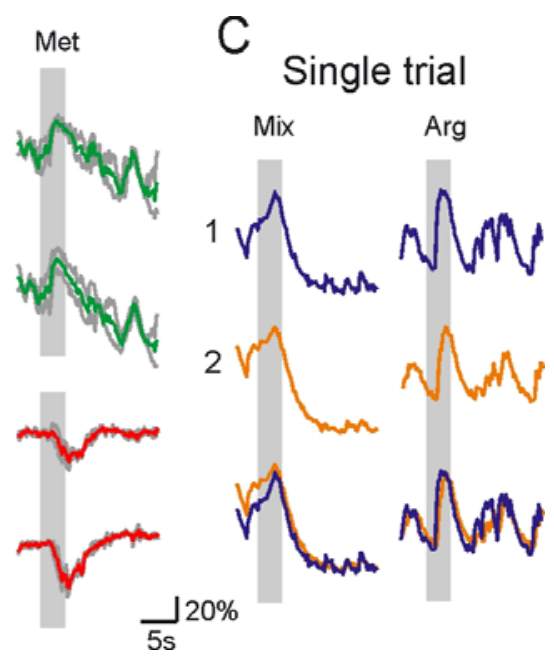

Fig. 3-26 Precisely matched responses of synchronous neurons to different odors

(A) Two pairs of synchronous M/T cells (top) and their spontaneous $\left[\mathrm{Ca}^{2+}\right]$ signals (bottom). Scale bar, $10 \mu \mathrm{m}$ (B) The responses of the four neurons to four different odorants (amino acid mixture, lysine, arginine and methionine, $50 \mu \mathrm{M}$ ). Color traces show average responses, gray traces show individual trials. (C) Single trial responses of a synchronous pair and their overlay. 
I performed the previously described experiments in a large number of $\mathrm{M} / \mathrm{T}$ cells. This allows me to reconstruct ensemble representations of different odors by population of M/T neurons. Fig. 3-27 shows the representation of 5 different odors by an ensemble of $34 \mathrm{M} / \mathrm{T}$ neurons (upper row). The response of each neuron was shown as a color coded bar with red showing excitatory responses and blue showing inhibitory responses. From this representation, it can be seen that each odor was represented by a specific pattern of excitation/inhibition distributed across the population. For each of these $34 \mathrm{M} / \mathrm{T}$ cells, I identified their synchronous partners and measured their odor responses. This allowed me to reconstruct the odor representation by a different ensemble of $34 \mathrm{M} / \mathrm{T}$ cells with each of them being synchronous to the corresponding neuron in the first ensemble (Fig. 3-27, lower row). The ensemble odor representation by these two non-overlapping groups of neurons showed a very high similarity (correlation coefficient $\mathrm{r}=0.87 \pm 0.02 ; \mathrm{n}=5$ odors). This high correlation was not due to a lack of specificity of the $\left[\mathrm{Ca}^{2+}\right]$ response waveforms because random reshuffling of the cell indices eliminated the correlation ( $\mathrm{r}$ shuffled $=0.03 \pm 0.01$ ). Further, when the correlation was measured over short time windows $(250 \mathrm{~ms})$, the correlation was reliable before, during and after odor responses (Fig. 3-28). These data suggest that OB neurons transmit more than one copy of odor codes. Further, it suggests that higher brain centers could retrieve similar odor information using both or either of the synchronous neurons. 


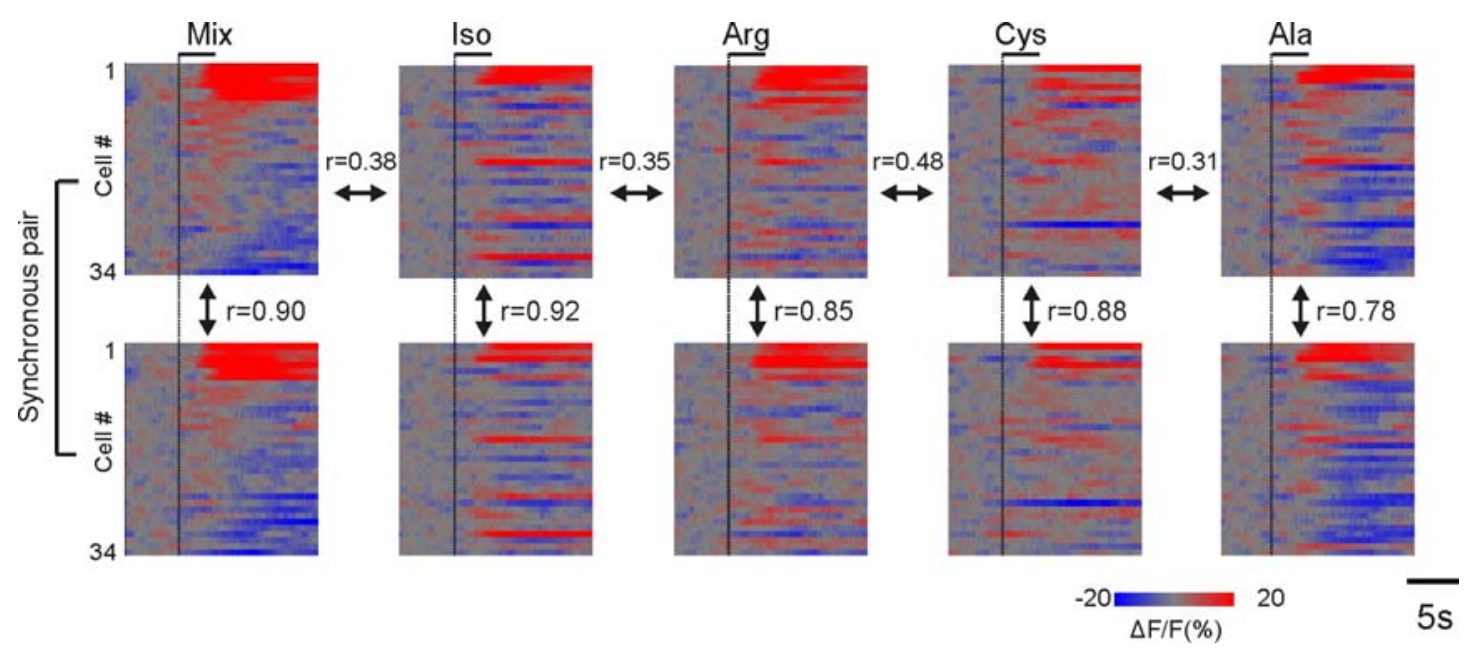

Fig. 3-27 Two copies of odor codes in the olfactory bulb. Responses of 34 neurons to five odors (upper row). The same odors are similarly represented by the synchronous partners of these neurons (lower row). r, correlation coefficient.

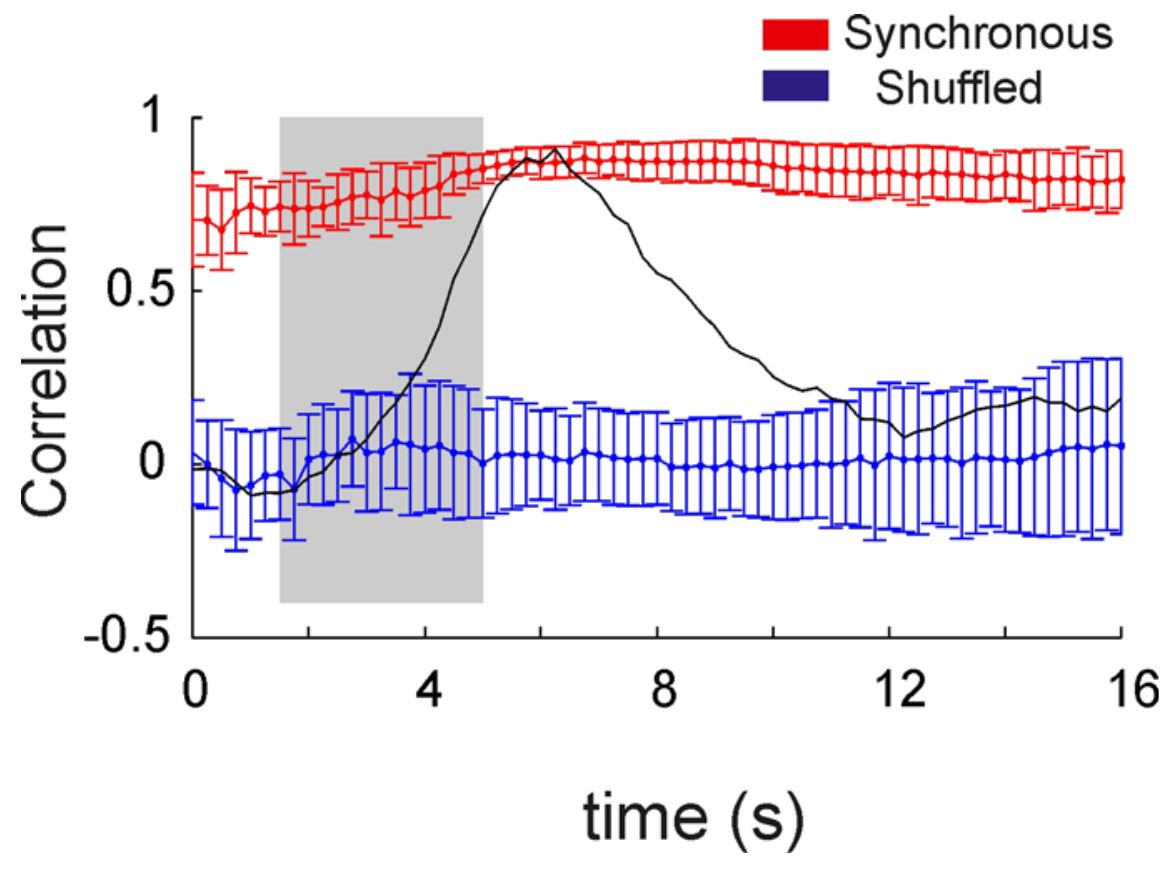

Fig. 3-28 Correlated activity remains high before, during and after odor responses. The correlation coefficient (r) between the two ensembles of neurons in Fig. 3-27 measured in successive 250ms windows plotted over time (red). Re-shuffling the cell order eliminates the correlation (blue). Error bars represents the s.e.m. of $r$ values over 12 trials ( 6 odors, each applied twice). Black trace shows the response waveform averaged over all neurons. 


\subsection{Synaptic mechanisms underlying correlated activity}

How can neurons of a synchronous module show such highly coordinated ongoing and odor-modulated activity? In other systems, correlated neuronal activity has been attributed to common excitatory inputs (Cobb et al., 1995;Alonso et al., 1996), synchronous inhibitory inputs (Gibson et al., 1999;Christie et al., 2005) or a direct coupling among neurons (Schoppa and Westbrook, 2002;Hayar et al., 2005; Christie et al., 2005). In this part of the thesis, I report some findings in which Dr. Bei-Jung Lin and I specifically examined these possibilities.

\subsubsection{Characterization of synaptic inputs and functional coupling}

We first analyzed patterns of synaptic inputs into synchronous neurons using duo whole cell patch-clamp that specifically target synchronous neurons. To separate between EPSCs and IPSCs, we hold the cells either at $-60 \mathrm{mV}$ (close to the chloride reversal potential) or at $0 \mathrm{mV}$ (close to the reversal potential for cationic currents). To minimize the potassium currents due to the opening of voltage-gated potassium channels at $0 \mathrm{mV}$, we used a $\mathrm{Cs}^{+}$and TEA based internal solution (in $\mathrm{mM}, \mathrm{NaCl} 1$, $\mathrm{MgCl}_{2}$ 1, TEA-Cl 2, $\mathrm{CsCH}_{3} \mathrm{SO} 4$ 79, HEPES 10, EGTA 5, ATP 3, GTP 0.3).

Fig. 3-29 shows one example of such experiments. At $-60 \mathrm{mV}, \mathrm{M} / \mathrm{T}$ cells predominantly showed synaptic inward currents that can be blocked by CNQX and APV. In synchronous neurons, some of these EPSC events occurred concurrently in both cells (Fig. 3-29 A, asterisks) while others occurred in only one of the neurons (arrows). The cross-correlogram of the membrane currents had a weak peak near $\Delta \mathrm{t}=0$ $(r(0)=0.35 \pm 0.07 ; n=8)$, suggesting that the excitatory inputs of these neurons were partly but not completely synchronous. At a potential close to the reversal potential for ionotropic currents mediated by glutamate receptors $(0 \mathrm{mV}), \mathrm{M} / \mathrm{T}$ cells predominantly showed synaptic outward currents that were abolished by picrotoxin 
$(50 \mu \mathrm{M})$. Examining these currents revealed that the IPSC events rarely occurred simultaneously in synchronous neurons (Fig. 3-29 B). The cross-correlogram of the membrane currents did not show a detectable peak $(\mathrm{r}(0)=0.05 \pm 0.02 ; \mathrm{n}=4)$. These data are not consistent with the idea of common inhibitory inputs underlying the correlated activity. Moreover, they show that even for neurons connected to the same glomerulus, the excitatory inputs are not completely the same.

A
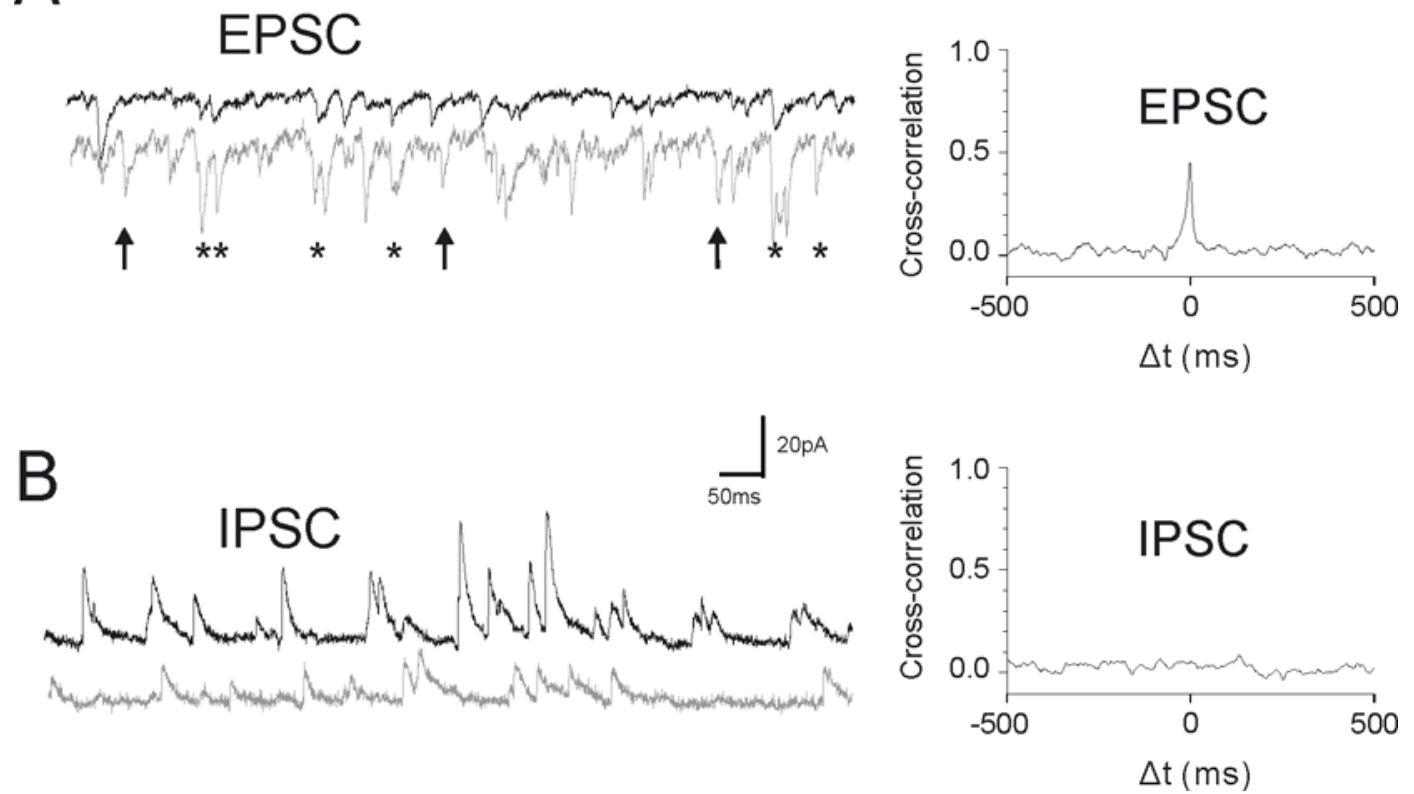

Fig. 3-29 Synaptic inputs recorded simultaneously in pairs of synchronous $M / T$ cells. (A) Left, excitatory synaptic currents (EPSC) in a pair of synchronous neurons measured at $-60 \mathrm{mV}$. Asterisks: synchronous events; arrows: asynchronous events. Right, membrane current cross-correlogram had a peak $(\mathrm{r}=0.43)$ near $\Delta \mathrm{t}=0$. (B) Left, inhibitory synaptic currents (IPSC) in synchronous neurons measured at $0 \mathrm{mV}$. Most IPSC events are not synchronous. Right, cross-correlogram of the IPSCs does not show a significant peak. Experiments performed by Dr. Bei-Jung Lin. 
We next examined the possibility of a direct coupling between synchronous $\mathrm{M} / \mathrm{T}$ cells. Previous studies in the mammalian OB show that M/T cells connected to the same glomerulus are coupled by gap junctions (Schoppa and Westbrook, 2002; Hayar et al., 2005; Christie et al., 2005). Because gap junctions can pass both depolarizing and hyperpolarizing currents, they could play a role in coordinating the excitatory/inhibitory responses. To test whether synchronous $\mathrm{M} / \mathrm{T}$ cells are electrically coupled, Dr. Bei-Jung Lin performed targeted paired whole-cell recording from these neurons.

In these experiments, a $25 \mathrm{mV}$ hyperpolarization pulse (from -60 to $-85 \mathrm{mV}$ ) applied to one cell reliably elicited a hyperpolarizing (outward) current in its synchronous partner (averaged amplitude: $3.73 \pm 0.51$ pA; $n=10$ pairs, Fig. 3-30 A). A current of similar amplitude was observed when the voltage pulses were applied into the other neuron (Fig. 3-30 A, right). The coupling current was not seen in recordings made in pairs of nearby but non-synchronous neurons $(n=2)$, suggesting that the coupling was specific. Finally, $\mathrm{Cd}^{2+}(200 \mu \mathrm{M})$, which blocked most synaptic currents, had little effect on the coupling current whereas the gap junction inhibitor carbenoxolone (200-400 $\mu \mathrm{M}$ ) largely reduced the current (Fig. 3-30 B, 80 $\pm 11 \%$ reduction, $n=4)$. These results show that the synchronous $\mathrm{M} / \mathrm{T}$ cells in the Xenopus OB are electrically coupled, suggesting that a glomerulus-specific M/T cell coupling is a general feature of glomerular function in the vertebrate $\mathrm{OB}$. 
A

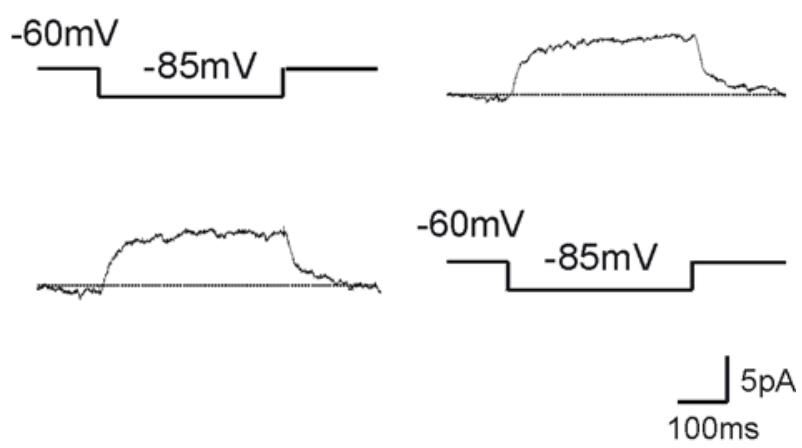

B

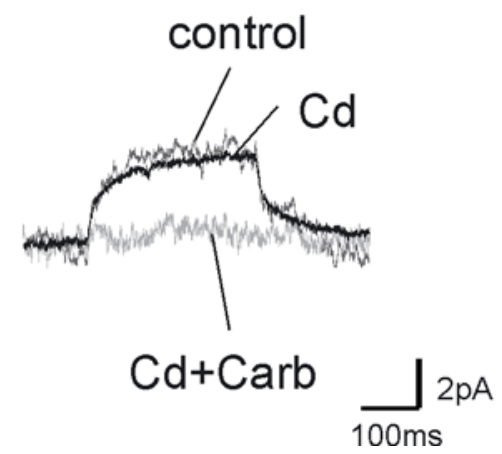

Fig. 3-30 Synchronous neurons are electrically coupled (A) Hyperpolarizing voltage steps ( -60 to $-85 \mathrm{mV}$ ) in one $\mathrm{M} / \mathrm{T}$ cell elicit hyperpolarizing (outward) currents in its synchronous partner (left column) and vice versa (right column). The current traces are averages of 80 sweeps. (B) The coupling current is not affected by $\mathrm{Cd}^{2+}$ but it is abolished by subsequent application of gap junction inhibitor carbenoxolone (Carb). Experiments performed by Dr. Bei-Jung Lin.

3.5.2 The effect of mutual coupling between synchronous neurons

Because Xenopus M/T cells have large input resistances (>1G $\Omega$ ) (Scheidweiler et al., 2001), the electrical coupling conductances we observed could have a strong functional effect. To test this possibility, I performed experiments in which I recorded one $\mathrm{M} / \mathrm{T}$ cell using whole cell patch clamp (Cell 1) while simultaneously monitoring the $\left[\mathrm{Ca}^{2+}\right]$ activity of its synchronous partner (Cell 2). In the current clamp mode, the patched clamped neuron (Cell 1) exhibits spontaneous fluctuation of membrane potentials, and this potential is locked to the spontaneous $\left[\mathrm{Ca}^{2+}\right]$ signals in its 
synchronous partner (Fig. 3-31). I further stimulated the patch clamped neuron while measuring the $\left[\mathrm{Ca}^{2+}\right]$ activity of other neurons. In these experiments, depolarizing a single $\mathrm{M} / \mathrm{T}$ cell induced measurable excitatory $\left[\mathrm{Ca}^{2+}\right]$ responses in its synchronous partner (but not in other cells in the same field of view, Fig. 3-32, n=4 synchronous pairs and 7 non-synchronous pairs). Moreover, hyperpolarizing the neuron reduced the spontaneous activity of its synchronous partner (Fig. 3-32), leading to a $\left[\mathrm{Ca}^{2+}\right]$ decrease similar to inhibitory odor-induced $\left[\mathrm{Ca}^{2+}\right]$ responses (see e.g. Fig. 3-24, right). These data show that a de- or hyperpolarization of an M/T cell can propagate and specifically modulate the responses of other synchronous neurons. This provides a mechanism to coordinate the responses of synchronous neurons during odor processing in the $\mathrm{OB}$.

\section{Current Clamp}
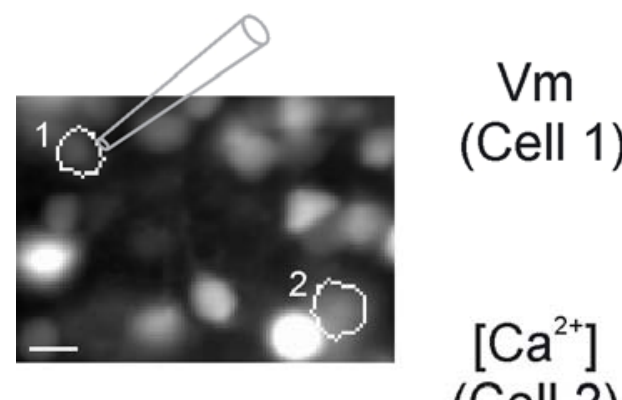

(Cell 2)
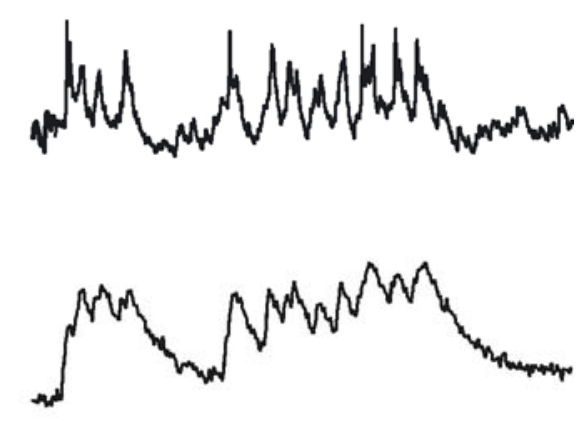

$\prod_{2 \mathrm{~s}} 5 \%, 10 \mathrm{mV}$

Fig. 3-31 Membrane potential of an $\mathrm{M} / \mathrm{T}$ cell time-locked to the $\left[\mathrm{Ca}^{2+}\right]$ transients of its synchronous partner. Simultaneous recording of membrane potential in a mitral cell (Cell1) during spontaneous $\left[\mathrm{Ca}^{2+}\right]$ activity of its synchronous partner (Cell2). The voltage in cell 1 is locked to the $\left[\mathrm{Ca}^{2+}\right]$ activity of cell 2 
A

\section{Voltage Clamp}

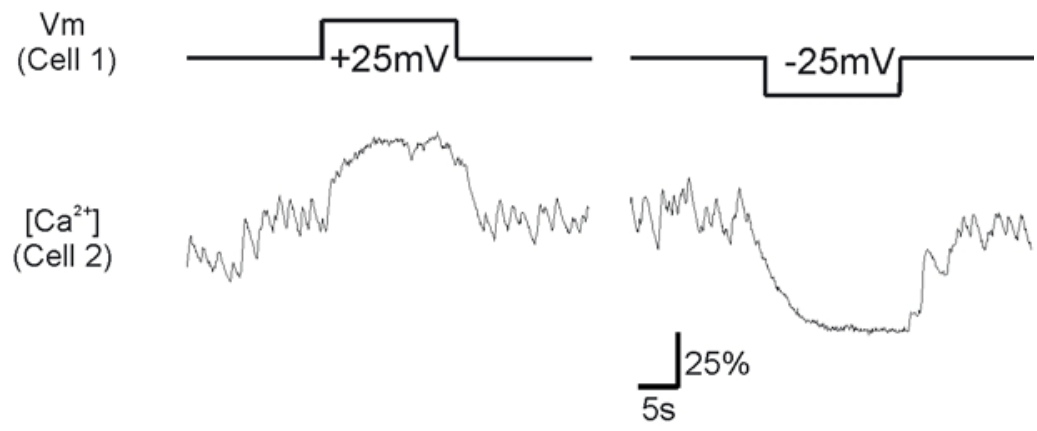

B
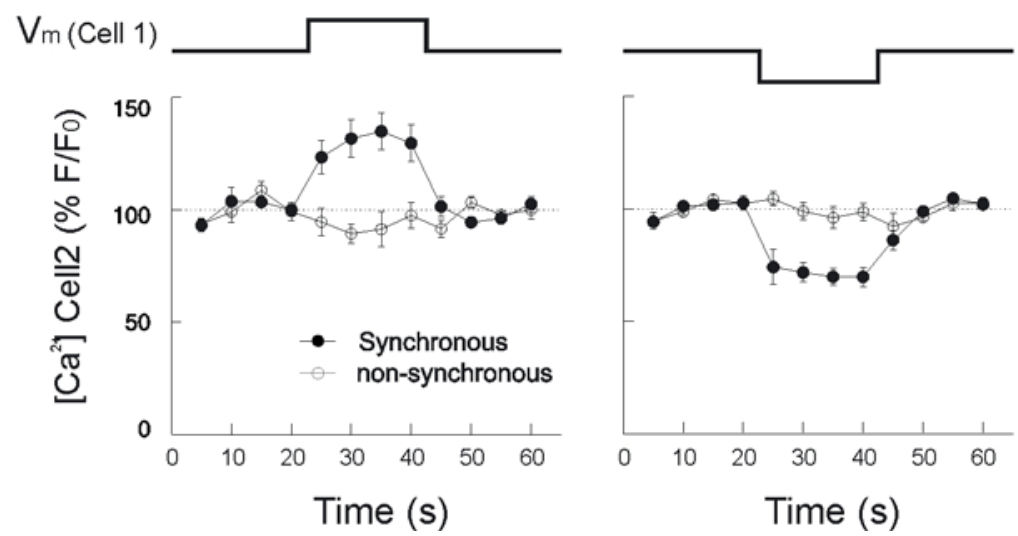

Fig. 3-32 Effects of the coupling between synchronous neurons (A) Stimulating a patch-clamped neuron (Cell1) in depolarizing or hyperpoloarizing direction specifically enhances or suppresses the $\left[\mathrm{Ca}^{2+}\right]$ activity of its synchronous partner (Cell2). (B) Summary (mean \pm s.e.m.) of the $\left[\mathrm{Ca}^{2+}\right]$ activity modulation in synchronous $(\mathrm{n}=4)$ and non-synchronous $(\mathrm{n}=7)$ pairs. 


\section{Discussion}

\subsection{Correlation maps for visualizing $\left[\mathrm{Ca}^{2+}\right]$ imaging data}

In Sec. 3.1, I introduce two correlation-based approaches to facilitate the visualization of time-dependent $\left[\mathrm{Ca}^{2+}\right]$ imaging data. The first approach, the NCM (neighborhood correlation map), labels all structures that exhibit activity during the recording period. The main advantage of the NCM is that it selectively labels active structures while excluding bright structures that do not exhibit any activity (Fig. 3-1). Furthermore, active structures can be clearly visualized even when they are not visible in the raw fluorescence image (Fig. 3-1). These properties make the NCM particularly appropriate for guiding the selection of regions of interests (Fig. 3-2).

There are several other approaches that could be used (or have been used) to visualize active structures in image time-series. For example, in the maximum projection map, each pixel is assigned the maximal intensity value of that pixel during the recording period. In this way, structures that become "brighter" at some point of the recording period will become more visible. However, the maximum projection map generally gives much worse contrast than the NCM because it does not reduce the labeling of bright but inactive structures (e.g. cell 2 in Fig. 3-1). Furthermore, structures showing a decrease in activity (i.e. become darker) will not be seen in the maximum projection map, but they can be clearly visualized in the NCM. Thus, for visualizing structures showing changes in fluorescence intensities, NCM is a preferred tool due to its high sensitivity and selectivity.

The second tool, the CCM (the cross-correlation map), labels structures that exhibit a particular reference waveform (Fig. 3-5). In general, one could take any 
$\mathrm{N}$-dimensional vector as the reference waveform (with $\mathrm{N}$ being the number of images). In this thesis I mostly used signals measured from a certain location of the image (e.g. $\left[\mathrm{Ca}^{2+}\right]$ signals at somata or dendrites) as references. Because correlation is an extremely sensitive way of detecting signals in noise (in the case of Gaussian noise, correlation has been proven to be the optimal way, Haykin, 1994), CCM reveals structures carrying the reference signals even when these structures are small and the signals are noisy (Fig. 3-5). This enables the visualization of fine neuronal processes of selected neurons even when these processes cannot be seen in the raw fluorescence image (Fig. 3-6 and Fig. 3-7).

To further illustrate the high sensitivity of CCM, I used a computer to simulate the process of fine structure detection using correlation. I first generated a noise-free data set that mimics $\left[\mathrm{Ca}^{2+}\right]$ signals in a thin dendritic structure (Fig. 4-1, upper row). I then added varying amount of noise to the data and generated CCMs using the noise-free signal as the reference waveform. Note that in all SNR levels shown, the signals of single pixels are so noisy that their similarity to the noise-free waveform can hardly be determined by visual inspection (Fig. 4-1). However, the presence of signals in these pixels can be clearly seen in the CCM. This further illustrates the high sensitivity of CCM to detect correlated structures in noisy conditions. 


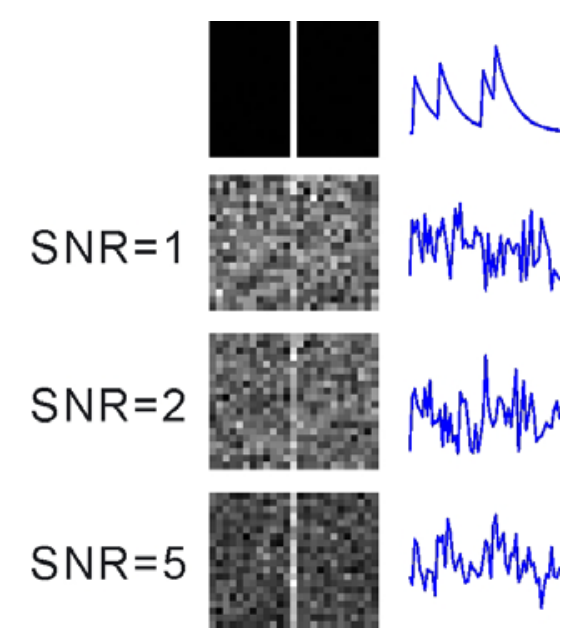

Fig. 4-1 Detecting fine structures using CCM under various SNR conditions. First row: A simulated noise-free data set that mimics $\left[\mathrm{Ca}^{2+}\right]$ imaging in a thin dendrite (white stripe in the middle). In these data, the white pixels exhibit a specific activity waveform (shown to the right) whereas the background pixels (black) carry no signal. Below: The CCMs obtained after adding random noise to the simulated data, using the noise free waveform as reference. The amount of noise is determined by the SNR values indicated to the left. The traces to the right of the CCMs show the signal of an example pixel after noise addition.

The CCM not only labels processes belonging to a given neuron but in some cases also labels other neurons that exhibit correlated activity (Fig. 3-10 and Fig. 3-22). In these two figures, the CCMs were generated using time-series of more than 600 images. Because the probability that any two vectors with length 600 will exhibit some random correlation is extremely low, the CCM labels only the structures whose signals show highly significant correlation to the reference signal. In the case of M/T cell spontaneous activity, CCM only labels highly correlated neurons that connect to the same glomerulus (Fig. 3-22).

The high sensitivity and high specificity of the CCM make it an extremely powerful tool to detect the presence of a particular signal waveform. The principle of the CCM is similar to the in-situ hybridization technique. In in-situ hybridization, the 
specific binding between a "probe" RNA and RNA molecules in the tissue enables visualizing the spatial expression pattern of a particular gene. In CCM, the correlation between a reference signal and each pixel's signal waveform enables visualizing the spatial "expression pattern" of a particular activity waveform in the recorded field of view. Interestingly, these two techniques can reveal different but complementary aspects of olfactory system function. In a sequence of classical findings (Ressler et al., 1994;Vassar et al., 1994;Mombaerts et al., 1996), the authors used in-situ hybridization techniques to map the expression pattern of a single olfactory receptor (OR) gene in the olfactory epithelium and the OB. These authors found that the expression of a given OR gene is confined to a subset of olfactory sensory neurons and that these neurons project their axons into the same glomerulus (Fig. 4-2, A). In this thesis, we used CCM to visualize the spatial "expression pattern" of a specific activity waveform in the OB. The resulting map reveals that a specific activity is found only in a subset of $\mathrm{M} / \mathrm{T}$ cells, and that these $\mathrm{M} / \mathrm{T}$ cells project their dendrites into the same glomerulus (Fig. 4-2, B). Thus, at the input level, an individual glomerulus collects convergent inputs from OSN expressing the same OR. At the output level, an individual glomerulus confers highly similar output activity to a specific ensemble of $\mathrm{M} / \mathrm{T}$ cells connected to it. This output function of the glomeruli is revealed here, for the first time, using a "digital version" of the in-situ hybridization technique (Fig. 4-2, B). 
OSN expressing the same OR gene converge to the same glomerulus

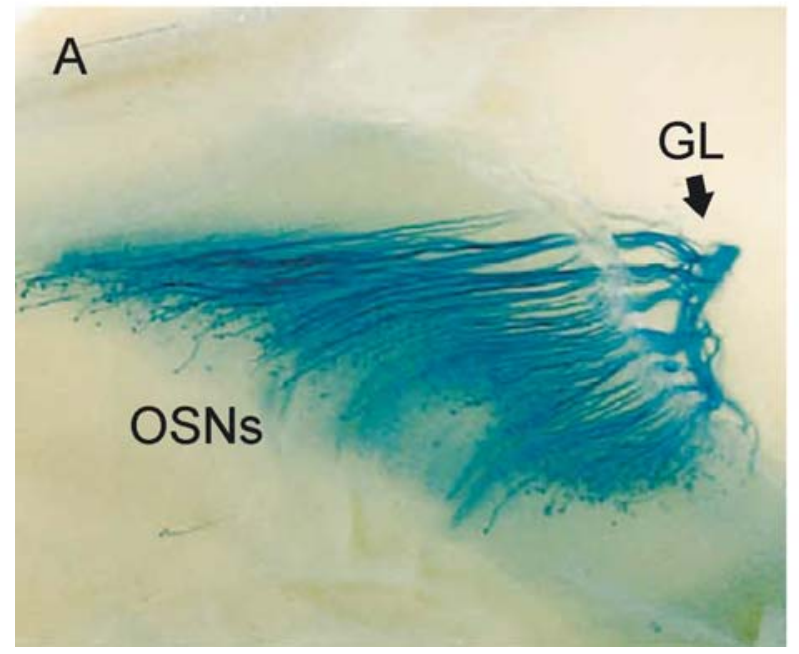

$\mathrm{M} / \mathrm{T}$ cells "expressing" the same activity diverge from the same glomerulus

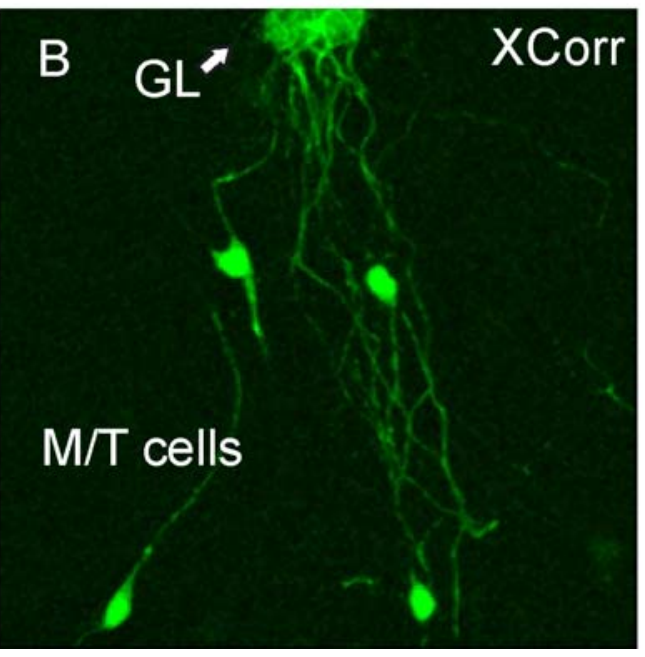

Fig. 4-2 (A) Projection patterns of OSNs expressing the olfactory receptor gene P2. The axons of these neurons converge to the same glomerulus. Image taken from (Mombaerts et al., 1996) (B) Projection patterns of M/T cells showing correlated activity waveform visualized using CCM. The dendrites of these neurons come from the same glomerulus (see also Fig. 3-22). 


\subsection{Spontaneous activity in the olfactory bulb}

Neurons in many brain regions often generate patterns of spontaneous activity in the absence of any stimulation. The spontaneous activity is traditionally considered to be random "noise" that has to be "averaged out" to yield a meaningful, sensory-related response (Raichle, 2006). However, recently studies start to uncover novel functional roles of neuronal spontaneous activity (Raichle, 2006). First, several studies have shown that the spontaneous activity in the cortex is not random but instead exhibits highly organized spatiotemporal structures (Kenet et al., 2003; Cossart et al., 2003;Ikegaya et al., 2004). Furthermore, intrinsic fluctuations of network "states" can play a role in modulating a neuron's sensory-induced responses (Petersen et al., 2003). These studies challenge the traditional view and place the intrinsic activity of neurons as an integral and important part of brain function.

The spontaneous activity of olfactory projecting neurons has been reported and analyzed in a number of different species. In-vivo recordings in the mammalian OB revealed that $\mathrm{M} / \mathrm{T}$ cells are spontaneously active at a rate between $0 \sim 30 \mathrm{~Hz}$, with an average background activity higher in awake than in anesthetized animals (Davison and Katz, 2007). In locusts, the baseline activity of antennal lobe projecting neurons (insect analog of the vertebrate $\mathrm{M} / \mathrm{T}$ cells) are abundant and are higher than downstream neurons in the mushroom body (Perez-Orive et al., 2002). However, despite these general descriptions, few studies have examined the spatiotemporal structures of spontaneous activity in populations of OB neurons.

In this thesis, I extensively analyze the spontaneous activity of many $\mathrm{OB}$ neurons using $\left[\mathrm{Ca}^{2+}\right]$ imaging. The main finding is that $\mathrm{M} / \mathrm{T}$ cells are organized into many "modules" of neurons each showing highly synchronous spontaneous activity 
(Fig. 3-10). Synchronous neurons are sparsely distributed in space and are intermingled with neurons belonging to other synchronous modules (Fig. 3-11). Moreover, the correlated spiking of these neurons is precise at a millisecond time resolution (Fig. 3-16, Fig. 3-17) and remains highly stable over time. These results provide direct evidences that the spontaneous activity of $\mathrm{OB}$ neurons exhibits highly organized dynamical structures.

Morphological reconstructions of synchronous neurons reveal that these neurons invariably connect to the same glomerulus (Fig. 3-19). In some cases, the glomerulus is the only place where the processes of the two neurons come close together (Fig. 3-20). This suggests a role of the glomerulus in synchronizing M/T cell activity. In the mammalian OB, correlated activity in pairs of mitral cells has been analyzed together with the neurons' glomerular connectivity. In these studies, electrical stimulation of olfactory nerves or intracellular injection of depolarizing current elicits sub-threshold or spiking activity that is synchronous only in neurons of the same glomerulus (Schoppa and Westbrook, 2001;Schoppa and Westbrook, 2002). Our results extend these findings and show that glomerulus-specific neurons can synchronize their activity even in the complete absence of any external stimulation.

What could be the function of the highly correlated spontaneous activity in $\mathrm{M} / \mathrm{T}$ cells of the same glomerulus? As $\mathrm{M} / \mathrm{T}$ cells connected to the same glomerulus can be identified from their synchronous ongoing activity, the synchronous activity provides a tonic "identity signal", which may allow downstream neuronal circuits to infer the common glomerular specificity of incoming axons without requiring odorant exposure. This may be important for the establishment of specific circuit connections in higher olfactory centers (Zou et al., 2001). In addition, correlated activity of M/T cells could play a role for the insertion of newborn granule cells, which continuously 
migrate into the $\mathrm{OB}$ and have to make specific connections with $\mathrm{M} / \mathrm{T}$ cells (Carleton et al., 2003).

\subsection{Odor coding by synchronous $\mathrm{M} / \mathrm{T}$ cell modules}

\subsubsection{Odor responses in glomerulus-specific neurons}

In Sec. 3.4, I analyze the response properties of M/T cells with respect to their glomerular connectivity as inferred from the correlation between the neurons' spontaneous activity. The main finding is that synchronous neurons (i.e. neurons of the same glomerulus) show precisely matched excitatory/inhibitory odor responses irrespective of their distances, whereas non-synchronous neurons (i.e. neurons of different glomeruli) can show very different responses even when they are located next to each other (Fig. 3-24, Fig. 3-25). These results suggest a precise relationship between the odor responses of $\mathrm{M} / \mathrm{T}$ cells and their glomerular connectivity.

The odor responses of $\mathrm{M} / \mathrm{T}$ cells have been extensively analyzed in a number of different species. However, due to technical difficulty, few studies have addressed how the responses of $\mathrm{M} / \mathrm{T}$ cells can be related to their glomerular connectivity. In the mammalian $\mathrm{OB}$, two different groups have attempted to address this question by recording pairs of mitral cells using microelectrodes and analyzing how the odor responses of the recorded neurons depend on the distances between them (Buonviso and Chaput, 1990;Buonviso et al., 1992;Egana et al., 2005). These studies made a critical assumption that nearby neurons have higher probabilities of coming from the same glomerulus, whereas distant neurons are less likely to connect to the same glomerulus. The first group (Buonviso and Chaput, 1990;Buonviso et al., 1992) reported some differences in response similarity of short or long distance pairs. They show that nearby pairs tend to respond to odors in a more similar way than distant pairs (Buonviso and Chaput, 1990;Buonviso et al., 1992). However, a recent study 
from a different group reported a somewhat contradictory result (Egana et al., 2005). They found that even nearby mitral cells show negligible synchronous activity and frequently exhibit dissimilar odor-induced responses (Egana et al., 2005). Based on these observations, these two groups reached very different conclusions concerning the coding function of neurons of the same glomerulus. However, despite this apparent difference, both groups pointed out that the spatial organization of mitral cell response properties is substantially different from that in the primary visual cortex where neurons' tuning properties vary orderly and smoothly according to their spatial locations (Ohki et al., 2005;Ohki et al., 2006). In mitral cells, both groups show that even the nearest neurons can often show substantially different odor-induced responses (Buonviso and Chaput, 1990;Buonviso et al., 1992;Egana et al., 2005). This conclusion is consistent to what we have observed here for Xenopus M/T cells (Fig. 3-23 and Fig. 3-24).

The studies discussed in the above paragraph based their conclusions on the assumption that nearby neurons have a higher probability connecting to the same glomerulus. However, this probability is very low even for closely located neurons. In the mice $\mathrm{OB}$, careful anatomical tracing revealed that $>80 \%$ of nearby $\mathrm{M} / \mathrm{T}$ cell pairs (distances $<40 \mu \mathrm{m}$ ) connect to different glomeruli (Urban and Sakmann, 2002). Thus, only a small fraction of nearby neurons actually connects to the same glomerulus. It is therefore unclear whether the response heterogeneity of nearby mitral cells reported in those studies reflects a heterogeneous connectivity pattern or a true difference in the responses of neurons of the same glomerulus.

Recently, a number of studies have recorded the activity of mitral cell pairs using duo whole-cell patch-clamp in in-vitro slice preparations of the rodent $\mathrm{OB}$ (Schoppa and Westbrook, 2001;Urban and Sakmann, 2002;Schoppa and Westbrook, 
2002; Christie et al., 2005). By putting fluorescent tracers into the recording pipettes, the authors can reconstruct the entire dendritic morphology of the recorded cell pairs and precisely determine whether the recorded neurons connect to the same or different glomeruli. They found that upon intra-cellular current injections or olfactory nerve stimulations, mitral cell pairs show spiking or subthreshold oscillatory responses that are synchronous only in cells that connect to the same glomerulus (Schoppa and Westbrook, 2001;Schoppa and Westbrook, 2002). This suggests a precise rule that relate mitral cells' activity patterns and their glomerular connectivity. However, because these studies are performed in an isolated OB preparation that does not preserve the connections from olfactory sensory neurons, they cannot address whether a similarly precise rule could be found during stimulations with natural odors.

The responses of mitral cells to natural odor inputs can be substantially more complex than responses induced by nerve stimulations or intracellular current injections. First of all, odor-stimulations activate a highly specific subset of olfactory sensory neurons in contrast to a relatively uniform and unspecific activation during electrical stimulations of the olfactory nerves. Furthermore, odor stimulations activate OB's inhibitory networks that mediate temporal patterning and inhibitory responses in mitral/tufted cells (Friedrich and Laurent, 2001;Friedrich et al., 2004;Yaksi et al., 2007; Lin et al., 2007). These complex responses patterns also cannot be mimicked by simple current injections. Thus, how odor stimulations modulate the responses in neurons connected to the same or different glomeruli have remained unknown.

Based on the circuitry of the $\mathrm{OB}$, there are several possible answers to this question. Because M/T cells of the same glomerulus receive inputs from OSN expressing the same OR type (Mombaerts et al., 1996), it is possible that the activity of these neurons are similarly modulated by odors. However, OSN inputs are not the 
only inputs to $\mathrm{M} / \mathrm{T}$ cells. It has been shown that the activity of $\mathrm{M} / \mathrm{T}$ cells also strongly depend on inputs from local interneurons (Isaacson and Strowbridge, 1998;Chen et al., 2000), top-down projections (Price and Powell, 1970;Jahr and Nicoll, 1982), and from other M/T cells (Urban and Sakmann, 2002;Schoppa and Westbrook, 2002). How these non-OSN inputs modulate $\mathrm{M} / \mathrm{T}$ cell responses and how such modulations can be related to the neurons' glomerular connectivity remains unknown. In one possibility, network processing can substantially modify the responses of $\mathrm{M} / \mathrm{T}$ cells from their initial OSN inputs (Wilson et al., 2004), and this modification could be different for each neuron of the same glomerulus (Urban and Sakmann, 2002;Brody and Hopfield, 2003;Egana et al., 2005). This would reduce the initial, afferent-defined similarity (Friedrich and Laurent, 2001; Yaksi et al., 2007) and lead to a variety of distinct responses of glomerulus-specific M/T cells (Egana et al., 2005). Alternatively, different $\mathrm{M} / \mathrm{T}$ cells of the same glomerulus might process their inputs in a coordinated way. In this view, glomerulus-specific neurons get similar OSN inputs and generate a coordinated output (Buonviso and Chaput, 1990;Schoppa and Westbrook, 2001;Schoppa and Westbrook, 2002), though being modulated by complex synaptic interactions. Thus, even under complex synaptic processing, M/T cells of the same glomerulus could still generate a highly coherent output (Buonviso and Chaput, 1990;Schoppa and Westbrook, 2001;Schoppa and Westbrook, 2002). Which of these fundamentally different coding strategies are implemented by OB's circuitry remains unknown.

In this thesis, we specifically examine this question by recording the odor-induced M/T cell responses in a nose-brain preparation of the Xenopus OB. The responses of these neurons clearly show both excitatory and inhibitory components (Fig. 3-23), suggesting that inhibitory circuits of this preparation are functional and 
contribute to the responses of $\mathrm{M} / \mathrm{T}$ cells. However, even with these network modulations, we observe a precisely matched response in M/T cells of the same glomerulus (i.e. in neurons showing synchronous spontaneous activity), both during excitatory and inhibitory responses (Fig. 3-24, Fig. 3-25). This data is consistent with the second model, suggesting that $\mathrm{M} / \mathrm{T}$ cells of the same glomerulus show precisely matched odor-induced responses even under complex synaptic processing of the OB network.

\subsubsection{Implications for odor coding}

What is the functional consequence of a highly similar response in M/T cells of the same glomerulus? One immediate implication is that it largely reduces the number of independent variables needed to describe the OB output. Usually, to completely describe a neural system containing $\mathrm{N}$ neurons, one would need $\mathrm{N}$ variables each representing the activity of a neuron. Thus, the neural representation of each odor can be considered as a vector in an N-dimensional "coding space" (Laurent, 2002), with $\mathrm{N}$ being the total number of $\mathrm{M} / \mathrm{T}$ cells. Because $\mathrm{M} / \mathrm{T}$ cells greatly outnumber glomeruli (by a factor of 3 in Drosophila, $\sim 5-10$ in lower vertebrates and 20-50 in mammals), the "coding space" at the M/T cell level is generally considered to be much larger than the initial glomerular feature space. One proposed function of such an enlarged space is that it increases the "distance" between the representations of similar odors, therefore enhancing their discrimination by downstream neurons (Friedrich and Laurent, 2001;Laurent, 2002). However, this theory has not received solid experimental support.

Our results in fact argue against the theory that the dimensionality of odor coding space increase substantially from glomerular to the M/T cell level. We show that $\mathrm{M} / \mathrm{T}$ cells diverging from the same glomerulus (i.e. synchronous neurons) show 
highly similar responses to all test odors (Fig. 3-26). Moreover, the responses of these neurons are similar not only in amplitudes, directions but also in the temporal patterns of the responses (Fig. 3-24 and Fig. 3-25). These results suggest that one could consider neurons of the same glomerulus as carrying a common odorant feature. Thus, the anatomical divergence from glomerulus to $\mathrm{M} / \mathrm{T}$ cells does not increase the number of independent variables, or "features" for odor coding, suggesting that the dimensionality of $\mathrm{M} / \mathrm{T}$ cell coding space stays the same as the initial glomerular feature space.

If the divergence from glomerulus to $\mathrm{M} / \mathrm{T}$ cells does not increase the dimensionality of odor coding space, what could be the function of such divergence? One possibility is that $\mathrm{M} / \mathrm{T}$ cells diverging from the same glomerulus serve as a "broadcasting channel" that carries highly similar information to downstream brain regions. Individual glomeruli receive convergent inputs from olfactory sensory neurons expressing the same olfactory receptor type (Mombaerts et al., 1996). After processing by OB's neuronal circuit, this sensory information is carried by $\mathrm{M} / \mathrm{T}$ cell axons to a number of higher brain centers including cortical areas and subcortical nuclei that mediate learning, memory and the emotional responses to odors. The OB being the interface between OSNs and central brain areas has to guarantee that the odor information is sent in parallel to various target areas. In this respect, a highly similar response in glomerulus-specific M/T cells appears to be well suitable for this goal. Our results further suggest that higher brain regions downstream to a given glomerulus receive temporally correlated inputs. This may contribute to the coordination between different brain regions involved in conscious perception, memory and the emotional responses of odors. 


\subsection{Mechanism of correlated activity}

In Sec. 3.5, we analyze various possible mechanisms for the correlated activity in neurons of the same glomerulus (i.e. synchronous neurons). The main conclusion is that these neurons show partially correlated EPSCs, non-correlated IPSCs and are coupled by a gap-junction mediated electrical conductance (Fig. 3-29, Fig. 3-30). Moreover, the electrical coupling has a strong effect such that stimulating a single M/T cell elicits specific responses in its synchronous partners (Fig. 3-32) but not in other $\mathrm{M} / \mathrm{T}$ cells.

The most plausible explanation for a correlated EPSC in synchronous M/T cells (Fig. 3-29) is a common input from olfactory sensory neurons. Because the dendritic fields of synchronous neurons overlap extensively within the glomerulus (Fig. 3-19, Fig. 3-20), it is possible that a given OSN axon synapses simultaneously with both M/T cells (Fig. 4-3, A). Activation of this OSN would then lead to simultaneous EPSC of the two M/T cells. An alternative explanation would be that an OSN axon synapses with the dendrite of one M/T cells (M1). The EPSCs then pass through nearby gap junctions and are detected in the other neuron (M2, Fig. 4-3, B). In this scenario, the currents detected in the "indirectly" connected neuron (M2) will be substantially smaller than the currents detected in the "directly" connected neuron (M1). This is sometimes the case as synchronous EPSC events (asterisks in Fig. 3-29 A) can sometimes be larger in one cell compared to the other cell. 


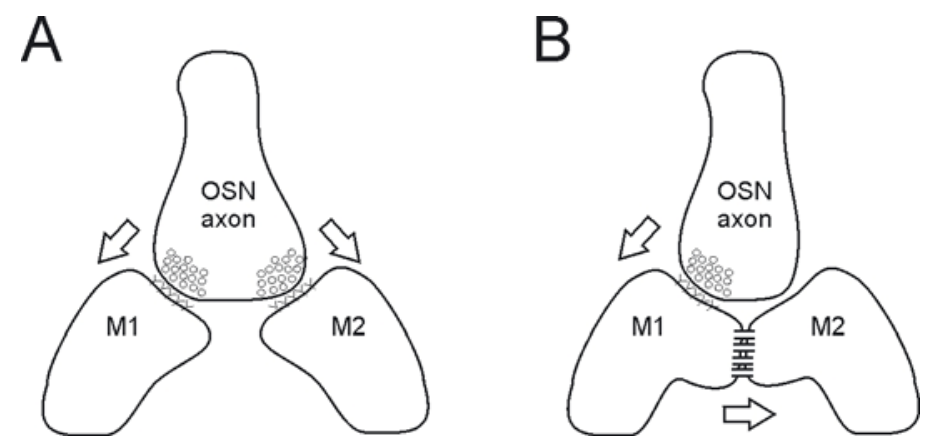

Fig. 4-3 Possible mechanisms for correlated EPSC events. (A) An OSN axon synapses directly with the dendrites of both M/T cells. Activation of this OSN would lead to synchronous EPSC of the two neurons. (B) An OSN axon synapses with only one M/T cell (M1). The EPSC then pass through nearby gap junctions and are simultaneously detected in the other M/T cell (M2).

At $0 \mathrm{mV}$, the spontaneous IPSC events in synchronous neurons show virtually no correlation (Fig. 3-29 B). This suggests that inhibitory interneurons rarely connect simultaneously with both $\mathrm{M} / \mathrm{T}$ cells. A major type of OB inhibitory interneurons, the granule cells, make synapse with $\mathrm{M} / \mathrm{T}$ cells mainly on their secondary (lateral) dendrites (Lledo et al., 2005). Because the secondary dendrites of synchronous M/T cells are often widely separated from each other (Fig. 3-19, Fig. 3-20), the probability that a granule cell make synapse simultaneously with two M/T cells is low. However, our results do not completely exclude such a possibility. For example, some inhibitory neurons might contact both $\mathrm{M} / \mathrm{T}$ cells but remain completely "silent" during the recording period. Activation of these neurons (e.g. by odor stimulations) might lead to correlated IPSCs and further enhances the correlated activity of synchronous neurons. However, in the absence of stimulations, IPSPs appear to play a minor role in synchronizing the spontaneous activity of $\mathrm{M} / \mathrm{T}$ cells.

Previous studies in the mammalian OB have revealed a specific electrical coupling in mitral cells of the same glomerulus (Schoppa and Westbrook, 2002; Christie et al., 2005). Our finding shows that synchronous M/T cells in the 
Xenopus OB are electrically coupled (Fig. 3-30), suggesting that a glomerulus-specific electrical coupling is a general feature of glomerular function in the vertebrate OB. The coupling conductance between synchronous neurons is $150 \pm 20 \mathrm{pS}$ (range: $48-256 \mathrm{pS}, \mathrm{n}=10$ pairs). This value is likely to be higher at the site of coupling (presumably within the glomerulus) because our recordings were made at the somata, approximately $100 \mu \mathrm{m}$ away from the glomerulus. As Xenopus M/T cells have small somata ( $\sim 10 \mu \mathrm{m}$ diameter) and very large input resistences $(>1 \mathrm{G} \Omega)$, a coupling of this size can have a strong functional effect. This is illustrated by the experiment shown in Fig. 3-32. In these experiments, a $25 \mathrm{mV}$ de- or hyper-polarization in a single $\mathrm{M} / \mathrm{T}$ cell can induce measurable $\left[\mathrm{Ca}^{2+}\right]$ responses in its synchronous partner. Considering a coupling conductance of $200 \mathrm{pS}$ and an input resistance of $1.5 \mathrm{G} \Omega$, a $25 \mathrm{mV}$ membrane potential change in the patch clamped neuron would lead to a $\sim 5.8 \mathrm{mV}$ membrane potential change in the coupled neurons. This could substantially increase or decrease the excitability of the imaged neuron (depending on stimulus directions) and lead to the change in $\left[\mathrm{Ca}^{2+}\right]$ activity (reflecting the rate of spontaneous firing) we observed in Fig. 3-32. 


\section{Summary}

In this study, we have used the OB as a model neuronal network and investigated how individual neurons behave as they are embedded in a native neuronal circuit. In the first part of the study (Sec. 3.1), I introduce two complementary tools, NCM and CCM, for visualizing signals in population $\left[\mathrm{Ca}^{2+}\right]$ imaging data. These tools provide high contrast for visualizing neurons and their fine processes that are not visible in the raw fluorescence image. This allows a specific measurement of signals in fine dendritic compartments. Further, it permits a multi-color visualization of neuronal networks during functional imaging of the brain.

In the second part of the study (Sec. 3.2), I investigate the spatiotemporal structure of spontaneous multi-neuronal activity patterns of OB neurons. The main finding is that there are multiple "modules" of $\mathrm{M} / \mathrm{T}$ cells that exhibit highly synchronous spontaneous $\left[\mathrm{Ca}^{2+}\right]$ activity. Furthermore, neurons belonging to a synchronous module are sparsely located and are intermingled with neurons belonging to other modules. These results reveal a novel form of spatiotemporal coordination of neuronal activity distinctly different from the $\left[\mathrm{Ca}^{2+}\right]$ domains (Yuste et al., 1992) or $\left[\mathrm{Ca}^{2+}\right]$ waves (Feller et al., 1996; Garaschuk et al., 2000) reported in other neuronal networks.

I further investigate how the synchronous spontaneous activity can be related to the underlying circuitry of $\mathrm{OB}$ (Sec. 3.3). Using targeted patch-clamp dye injection as well as CCM in 3 dimensions, I reconstruct the dendritic connections of neurons showing synchronous activity. The main finding is that synchronous neurons invariably have dendrites that come from the same glomerulus. These results suggest a strikingly precise relationship between a coordinated $\mathrm{M} / \mathrm{T}$ cell activity and the 
underlying circuit architecture of the OB network. Further, it suggests an application of using the correlation of spontaneous $\left[\mathrm{Ca}^{2+}\right]$ activity as a tool for detecting neurons that connect to the same glomerulus.

In Sec. 3.4, we investigate how the odor representation of $\mathrm{M} / \mathrm{T}$ cells can be related to their glomerular connectivity, using the synchronous spontaneous activity of these neurons as a mean to infer their glomerular connections. The main finding is that synchronous neurons (i.e. neurons of the same glomerulus) show precisely matched excitatory/inhibitory odor-induced responses. These results provide the first direct evidence suggesting that neurons of the same glomerulus encode the same odorant feature. These results will be important for understanding how odors are coded by populations of $\mathrm{M} / \mathrm{T}$ cells.

In the final set of experiments (Sec. 3.5), we investigate the synaptic mechanisms underlying the coordinated activity. The main result is that synchronous neurons share partially correlated EPSCs, uncorrelated IPSCs and are coupled by gap junctions. Furthermore, stimulating a single $\mathrm{M} / \mathrm{T}$ cell induce measurable and specific $\left[\mathrm{Ca}^{2+}\right]$ responses in its synchronous partner. These results provide insights into the mechanisms of the synchronous activity.

In this thesis, we analyze the function of the $\mathrm{OB}$ as an integral neuronal network. It reveals a number of network level phenomena, their underlying synaptic mechanisms, and their functional significance for the coding and the processing of odor information. It may be hoped that these results as well as the approaches will contribute to our continuous quest for a link between the function of individual neurons and the function of the brain as a whole. 


\section{Reference List}

Alonso, J. M., W. M. Usrey, and R. C. Reid. 1996. Precisely correlated firing in cells of the lateral geniculate nucleus. Nature 383:815-819.

Brody, C. D. and J. J. Hopfield. 2003. Simple networks for spike-timing-based computation, with application to olfactory processing. Neuron 37:843-852.

Buonviso, N. and M. A. Chaput. 1990. Response similarity to odors in olfactory bulb output cells presumed to be connected to the same glomerulus: electrophysiological study using simultaneous single-unit recordings. $J$. Neurophysiol. 63:447-454.

Buonviso, N., M. A. Chaput, and F. Berthommier. 1992. Temporal pattern analyses in pairs of neighboring mitral cells. J. Neurophysiol. 68:417-424.

Buzsaki, G. 2004. Large-scale recording of neuronal ensembles. Nat. Neurosci. 7:446-451.

Cajal, R. 1899. Histology of the Nervous System of Man and the Vertebrates.

Cang, J. and J. S. Isaacson. 2003. In vivo whole-cell recording of odor-evoked synaptic transmission in the rat olfactory bulb. J. Neurosci. 23:4108-4116.

Carleton, A., L. T. Petreanu, R. Lansford, A. varez-Buylla, and P. M. Lledo. 2003. Becoming a new neuron in the adult olfactory bulb. Nat. Neurosci. 6:507-518.

Chen, T. W., B. J. Lin, E. Brunner, and D. Schild. 2006. In situ background estimation in quantitative fluorescence imaging. Biophysical Journal 90:2534-2547.

Chen, W. R., W. Xiong, and G. M. Shepherd. 2000. Analysis of relations between NMDA receptors and GABA release at olfactory bulb reciprocal synapses. Neuron 25:625-633.

Christie, J. M., C. Bark, S. G. Hormuzdi, I. Helbig, H. Monyer, and G. L. Westbrook. 2005. Connexin36 mediates spike synchrony in olfactory bulb glomeruli. Neuron 46:761-772.

Cinelli, A. R., K. A. Hamilton, and J. S. Kauer. 1995. Salamander olfactory bulb neuronal activity observed by video rate, voltage-sensitive dye imaging. III. Spatial and temporal properties of responses evoked by odorant stimulation. $J$. Neurophysiol. 73:2053-2071. 
Cobb, S. R., E. H. Buhl, K. Halasy, O. Paulsen, and P. Somogyi. 1995.

Synchronization of neuronal activity in hippocampus by individual GABAergic interneurons. Nature 378:75-78.

Cossart, R., D. Aronov, and R. Yuste. 2003. Attractor dynamics of network UP states in the neocortex. Nature 423:283-288.

Cowan W.M., Sudhof T.C, and Stevens C.F. 2003. Synapses. The Johns Hopkins University Press, Baltimore, Maryland.

Davison, I. G. and L. C. Katz. 2007. Sparse and selective odor coding by mitral/tufted neurons in the main olfactory bulb. J. Neurosci. 27:2091-2101.

Egana, J. I., M. L. Aylwin, and P. E. Maldonado. 2005. Odor response properties of neighboring mitral/tufted cells in the rat olfactory bulb. Neuroscience 134:1069-1080.

Feller, M. B., D. P. Wellis, D. Stellwagen, F. S. Werblin, and C. J. Shatz. 1996. Requirement for cholinergic synaptic transmission in the propagation of spontaneous retinal waves. Science 272:1182-1187.

Frahm, J. 1993. Nuclear magnetic resonance studies of human brain in vivo: anatomy, function, and metabolism. Adv. Exp. Med. Biol. 333:257-271.

Friedrich, R. W., C. J. Habermann, and G. Laurent. 2004. Multiplexing using synchrony in the zebrafish olfactory bulb. Nat. Neurosci. 7:862-871.

Friedrich, R. W. and G. Laurent. 2001. Dynamic optimization of odor representations by slow temporal patterning of mitral cell activity. Science 291:889-894.

Garaschuk, O., J. Linn, J. Eilers, and A. Konnerth. 2000. Large-scale oscillatory calcium waves in the immature cortex. Nat. Neurosci. 3:452-459.

Garaschuk, O., R. I. Milos, and A. Konnerth. 2006. Targeted bulk-loading of fluorescent indicators for two-photon brain imaging in vivo. Nat. Protoc. $1: 380-386$.

Gibson, J. R., M. Beierlein, and B. W. Connors. 1999. Two networks of electrically coupled inhibitory neurons in neocortex. Nature 402:75-79.

Greer, C. A. and G. M. Shepherd. 1998. Olfactory bulb. In Synaptic Organization of the Brain. G. M. Shepherd, editor. Oxford University Press, New York. 
Grynkiewicz, G., M. Poenie, and R. Y. Tsien. 1985. A new generation of Ca2+ indicators with greatly improved fluorescence properties. J. Biol. Chem. 260:3440-3450.

Hamilton, K. A. and J. S. Kauer. 1989. Patterns of intracellular potentials in salamander mitral/tufted cells in response to odor stimulation. J. Neurophysiol. 62:609-625.

Hayar, A., M. T. Shipley, and M. Ennis. 2005. Olfactory bulb external tufted cells are synchronized by multiple intraglomerular mechanisms. J. Neurosci. 25:8197-8208.

Haykin, S. 1994. Communication Systems. John Wiley \& Sons.

Hille B. 2001. Ion Channels of Excitable Membranes. Sinauer Associates, Sunderland, MA.

Ikegaya, Y., G. Aaron, R. Cossart, D. Aronov, I. Lampl, D. Ferster, and R. Yuste. 2004. Synfire chains and cortical songs: temporal modules of cortical activity. Science 304:559-564.

Isaacson, J. S. and B. W. Strowbridge. 1998. Olfactory reciprocal synapses: dendritic signaling in the CNS. Neuron 20:749-761.

Jahr, C. E. and R. A. Nicoll. 1982. Noradrenergic modulation of dendrodendritic inhibition in the olfactory bulb. Nature 297:227-229.

Kashiwadani, H., Y. F. Sasaki, N. Uchida, and K. Mori. 1999. Synchronized oscillatory discharges of mitral/tufted cells with different molecular receptive ranges in the rabbit olfactory bulb. J. Neurophysiol. 82:1786-1792.

Kenet, T., D. Bibitchkov, M. Tsodyks, A. Grinvald, and A. Arieli. 2003. Spontaneously emerging cortical representations of visual attributes. Nature 425:954-956.

Komiyama, T. and L. Luo. 2006. Development of wiring specificity in the olfactory system. Curr. Opin. Neurobiol. 16:67-73.

Laurent, G. 2002. Olfactory network dynamics and the coding of multidimensional signals. Nat. Rev. Neurosci. 3:884-895.

Lin, B. J., T. W. Chen, and D. Schild. 2007. Cell type-specific relationships between spiking and $[\mathrm{Ca} 2+] \mathrm{i}$ in neurons of the Xenopus tadpole olfactory bulb. $J$. 
Physiol 582:163-175.

Lledo, P. M., G. Gheusi, and J. D. Vincent. 2005. Information processing in the mammalian olfactory system. Physiol Rev. 85:281-317.

Luo, M. and L. C. Katz. 2001. Response correlation maps of neurons in the mammalian olfactory bulb. Neuron 32:1165-1179.

Manzini, I., C. Brase, T. W. Chen, and D. Schild. 2007. Response profiles to amino acid odorants of olfactory glomeruli in larval Xenopus laevis. J. Physiol 581:567-579.

Manzini, I., W. Rossler, and D. Schild. 2002. cAMP-independent responses of olfactory neurons in Xenopus laevis tadpoles and their projection onto olfactory bulb neurons. J. Physiol 545:475-484.

Mombaerts, P., F. Wang, C. Dulac, S. K. Chao, A. Nemes, M. Mendelsohn, J. Edmondson, and R. Axel. 1996. Visualizing an olfactory sensory map. Cell 87:675-686.

Mori, K., H. Nagao, and Y. Yoshihara. 1999. The olfactory bulb: coding and processing of odor molecule information. Science 286:711-715.

Murakami, M., H. Kashiwadani, Y. Kirino, and K. Mori. 2005. State-dependent sensory gating in olfactory cortex. Neuron 46:285-296.

Nagayama, S., S. Zeng, W. Xiong, M. L. Fletcher, A. V. Masurkar, D. J. Davis, V. A. Pieribone, and W. R. Chen. 2007. In vivo simultaneous tracing and Ca2+ imaging of local neuronal circuits. Neuron 53:789-803.

Nezlin, L. P. and D. Schild. 2000. Structure of the olfactory bulb in tadpoles of Xenopus laevis. Cell Tissue Res. 302:21-29.

Nieuwkoop, P. D. and Faber J. 1967. Normal Table of Xenopus laevis. North Holland, Amsterdam.

Ohki, K., S. Chung, Y. H. Ch'ng, P. Kara, and R. C. Reid. 2005. Functional imaging with cellular resolution reveals precise micro-architecture in visual cortex. Nature 433:597-603.

Ohki, K., S. Chung, P. Kara, M. Hubener, T. Bonhoeffer, and R. C. Reid. 2006. Highly ordered arrangement of single neurons in orientation pinwheels. Nature 442:925-928. 
Perez-Orive, J., O. Mazor, G. C. Turner, S. Cassenaer, R. I. Wilson, and G. Laurent. 2002. Oscillations and sparsening of odor representations in the mushroom body. Science 297:359-365.

Petersen, C. C., T. T. Hahn, M. Mehta, A. Grinvald, and B. Sakmann. 2003. Interaction of sensory responses with spontaneous depolarization in layer $2 / 3$ barrel cortex. Proc. Natl. Acad. Sci. U. S A 100:13638-13643.

Price, J. L. and T. P. Powell. 1970. An electron-microscopic study of the termination of the afferent fibres to the olfactory bulb from the cerebral hemisphere. J. Cell Sci. 7:157-187.

Raichle, M. E. 2006. Neuroscience. The brain's dark energy. Science 314:1249-1250.

Ressler, K. J., S. L. Sullivan, and L. B. Buck. 1994. Information coding in the olfactory system: evidence for a stereotyped and highly organized epitope map in the olfactory bulb. Cell 79:1245-1255.

Sato, T. R., N. W. Gray, Z. F. Mainen, and K. Svoboda. 2007. The Functional Microarchitecture of the Mouse Barrel Cortex. PLoS. Biol. 5:e189.

Scheidweiler, U., L. Nezlin, J. Rabba, B. Muller, and D. Schild. 2001. Slice culture of the olfactory bulb of Xenopus laevis tadpoles. Chem. Senses 26:399-407.

Schoppa, N. E. and G. L. Westbrook. 2001. Glomerulus-specific synchronization of mitral cells in the olfactory bulb. Neuron 31:639-651.

Schoppa, N. E. and G. L. Westbrook. 2002. AMPA autoreceptors drive correlated spiking in olfactory bulb glomeruli. Nat. Neurosci. 5:1194-1202.

Shepherd, G. M. 2005. Perception without a thalamus how does olfaction do it? Neuron 46:166-168.

Stosiek, C., O. Garaschuk, K. Holthoff, and A. Konnerth. 2003. In vivo two-photon calcium imaging of neuronal networks. Proceedings of the National Academy of Sciences of the United States of America 100:7319-7324.

Urban, N. N. and B. Sakmann. 2002. Reciprocal intraglomerular excitation and intraand interglomerular lateral inhibition between mouse olfactory bulb mitral cells. J. Physiol 542:355-367.

Vassar, R., S. K. Chao, R. Sitcheran, J. M. Nunez, L. B. Vosshall, and R. Axel. 1994. Topographic organization of sensory projections to the olfactory bulb. Cell 
79:981-991.

Wilson, R. I., G. C. Turner, and G. Laurent. 2004. Transformation of olfactory representations in the Drosophila antennal lobe. Science 303:366-370.

Yaksi, E. and R. W. Friedrich. 2006. Reconstruction of firing rate changes across neuronal populations by temporally deconvolved Ca2+ imaging. Nat. Methods 3:377-383.

Yaksi, E., B. Judkewitz, and R. W. Friedrich. 2007. Topological Reorganization of Odor Representations in the Olfactory Bulb. PLoS. Biol. 5:e178.

Yuste, R., A. Peinado, and L. C. Katz. 1992. Neuronal domains in developing neocortex. Science 257:665-669.

Zou, Z., L. F. Horowitz, J. P. Montmayeur, S. Snapper, and L. B. Buck. 2001. Genetic tracing reveals a stereotyped sensory map in the olfactory cortex. Nature 414:173-179. 


\section{Abbreviations}

3D

APV

BOLD

$\mathrm{CCM}$

CNQX

DMSO

EGTA

EPSC

fMRI

GL

HEPES

IPSC

$\mathrm{M} / \mathrm{T}$

NCM

OB

$\mathrm{OE}$

ON

OR

OSN

RNA

ROI

SD

SEM

SNR

TTX 3-dimensional

(2R)-amino-5-phosphonovaleric acid

blood oxygen level dependent

cross-correlation map

6-cyano-7-nitroquinoxaline-2,3-dione

Dimethyl sulfoxide

ethylene glycol tetraacetic acid

excitatory postsynaptic current

functional magnetic resonance imaging

glomerulus

4-(2-hydroxyethyl)-1-piperazineethanesulfonic acid inhibitory postsynaptic current

mitra/tufted

neighborhood correlation map

olfactory bulb

olfactory epithelium

olfactory nerve

olfactory receptor

olfactory sensory neuron

ribonucleic acid

region of interest

standard deviation

standard error of the mean

signal to noise ratio

tetrodotoxin 


\section{Acknowledgements}

I would like to express my deepest gratefulness to my supervisor Prof. Detlev Schild for giving me the opportunity to work on this project and for his constant supports and suggestions throughout these years.

I would like to thank my thesis committee members Prof. Walter Stühmer and Dr. Fred Wolf for many valuable and insightful comments and suggestions

I would like to thank the coordination office of the neuroscience program, especially Prof. Michael Hörner, Dr. Steffen Burkhardt, and Sandra Drube for many helps.

I would like to thank Dr. Ivan Manzini, Dr. Andre Zeug, Mrs. Gudrun Federkeil and all members of our lab for constant help and for an enjoyable and memorable time.

I would like to thank Torsten Nägel, Wilfried Mesecke, Jens Kowalski and all members of the amazing electrical/mechanical workshop for building equipments essential for the project. Without you, a large part of this work wouldn't have been possible.

I would like to thank Howard Schultens and Andrew Woehler for reading and correcting my manuscripts and thesis. These are really important helps for me!

I would like to thank all my friends in Göttingen, especially those in the neuroscience program and those in the Göttingen Taiwanese student society for all the great time together.

I would like to thank my dear parents and my dear brother for their unquestioning love and support. Talking to you on the phone every weekend is the most important and the happiest time for me.

Lastly, but most of all, I want to thank my wife Belle for many helps, supports and for sharing every difficult and happy moments with me. 


\section{Curriculum Vitae}

Name

Date of birth

Place of birth

Email

\section{$\underline{\text { Education }}$}

Since March 2005

March 2005

September 2004 - March 2005

Since September 2003

October 2001 - June 2003

June 2001

September 1997 - June 2001
Tsai-Wen Chen

July 4, 1979

Taipei, Taiwan

tsaiwen@gmail.com

PhD student in the laboratory of Prof. Schild, Department of Neurophysiology and Cellular Biophysics, University of Göttingen, Germany

MSc, Neuroscience, University of Göttingen

MSc student in the laboratory of Prof. Schild, Department of Neurophysiology and Cellular Biophysics, University of Göttingen, Germany

International MSc / PhD Program in

Neuroscience, Göttingen, Germany

Military service, Air Defense Missile

Command, ROC army, Taiwan

BSc, Electrical Engineering, National Taiwan University, Taipei, Taiwan

Department of Electrical Engineering, National Taiwan University, Taipei, Taiwan 


\section{$\underline{\text { Publication list }}$}

Chen, T. W., B. J. Lin, E. Brunner, and D. Schild. 2006. In situ background estimation in quantitative fluorescence imaging. Biophysical Journal 90:2534-2547

Manzini, I., C. Brase, T. W. Chen, and D. Schild. 2007. Response profiles to amino acid odorants of olfactory glomeruli in larval Xenopus laevis. J. Physiol 581:567-579.

Lin, B. J., T. W. Chen, and D. Schild. 2007. Cell type-specific relationships between spiking and $\left[\mathrm{Ca}^{2+}\right]_{\mathrm{i}}$ in neurons of the Xenopus tadpole olfactory bulb. J. Physiol 582:163-175.

Chen, T. W.*, B. J. Lin*, and D. Schild. Odor coding by modules of coherent mitral/tufted cells in the vertebrate olfactory bulb. Submitted. (*equal contribution)

Junek, S.*, T. W. Chen*, M. Alevra, and D. Schild. Activity correlation imaging: visualizing function and connectivity of neuronal population. Submitted. (*equal contribution) 